\title{
ON SPIN AND MODULARITY IN CONFORMAL FIELD THEORY
}

\author{
BY IGOR KRIZ ${ }^{1}$
}

ABSTRACT. - We shall investigate chiral conformal field theories with 1-dimensional conformal anomalies with or without spin. We shall rigorously classify such anomalies and describe their modularity properties. Thereby, we give a detailed mathematical treatment of the simplest case of G. Segal's approach to conformal field theory.

(c) 2003 Éditions scientifiques et médicales Elsevier SAS

RÉSUMÉ. - Nous étudions les théories du champ conforme chirales avec anomalies conformes de dimension 1, avec ou sans spin. Nous faisons une classification rigoureuse de telles anomalies et nous décrivons leurs propriétés modulaires. Nous donnons ainsi un fondement mathématique au cas le plus simple de l'approche de la théorie du champ conforme due à G. Segal.

(C) 2003 Éditions scientifiques et médicales Elsevier SAS

\section{Introduction}

This paper is about the relationship between conformal anomalies and modularity formulas for partition functions $Z(\tau)$ of holomorphic conformal field theories. Our goal is to work out rigorously a very basic case, namely when the anomaly is 1-dimensional. The author became interested in this question as a prerequisite for investigating connections between holomorphic conformal field theory and elliptic cohomology. (That project will be pursued elsewhere.) During the investigation of the question, a surprizing number of subtle complications appeared, which will turn out to be our main focus.

The mathematical definition of conformal field theory (CFT), and its anomaly, was given by Segal [21]. In this paper, Segal also gives a modularity formula for $Z(\tau)$ for holomorphic CFT's ([21], Proposition 6.9). Terminological note: In physics, holomorphic CFT's are also sometimes called chiral (and antiholomorphic antichiral), although the author believes originally chiral was a common term for both holomorphic and antiholomorphic CFT's. Segal's modularity formula says that after multiplying by a certain power of $q=\mathrm{e}^{2 \pi i \tau}$, depending on the central charge of the theory, $Z(\tau)$ transforms under modular transformations $g$ of level 1 by multiplication by a certain character of $S L_{2}(\mathbb{Z})$ (see also Corollary 3.4 below).

In [21], (Section 8), Segal constructs a series of basic examples of holomorphic conformal field theories with their anomalies (or, more precisely, slight generalizations). The space of states $\mathcal{F}\left(\Omega^{\alpha}\right)$ of these CFT's is the Hilbert completion of the exterior algebra on the Hilbert space of $\alpha$-forms on $S^{1}, \alpha \in \frac{1}{2} \mathbb{Z}$ (the Fock space - see Section 2 below). In some sense, these are the

\footnotetext{
${ }^{1}$ The author was supported by the NSF. 
most basic examples. In physics, they are known as $b-c$ systems (see [12,17], Chapter 13.1). The most interesting case is $\alpha=1 / 2$, which is the CFT of the chiral fermion. But in this case, we get a Spin-CFT, i.e. a mathematical object which satisfies the axiom of CFT when the space $\mathcal{C}$ of Riemann surfaces is replaced by the space $\mathcal{C}_{\text {spin }}$ of Riemann surfaces with Spin-structure (see Section 2 below). The formula [21], Proposition 6.9, does not apply as stated to that case. Of course, for this example, the partition function is known explicitly, but it would be nice to have a general formula which includes this case. When $\alpha \in \mathbb{Z}$, then [21], Proposition 6.9 does apply, but finds that $Z(\tau)=0$ (these theories are $\mathbb{Z} / 2$-graded, causing cancellation of terms - see Section 2 below).

The goal of this note is to find modularity formula which would apply, in a non-trivial way, to a general class of conformal field theories with 1-dimensional anomalies, including the theories $\mathcal{F}\left(\Omega^{\alpha}\right), \alpha \in \frac{1}{2} \mathbb{Z}$. We use the class of all Spin-conformal field theories with 1-dimensional anomaly in the case when $\alpha \in \mathbb{Z}+\frac{1}{2}$. When $\alpha \in \mathbb{Z}$, spin is not relevant, but the theories $\mathcal{F}\left(\Omega^{\alpha}\right)$ do have extra structure, namely can be defined on the space $\mathcal{C}_{\mathbb{Z} / 2}$ of Riemann surfaces equipped with a $\mathbb{Z} / 2$-principal bundle $L$ (which we call a $S q r t$-structure). This allows one to define another partition function $Z^{-}(\tau)$ which is non-zero for $\mathcal{F}\left(\Omega^{\alpha}\right)$. We obtain a general modularity formula for the partition functions $Z^{-}(\tau)$.

In the proof of Segal's formula [21], Proposition 6.9, the major ingredient is a theorem of Mumford, which classifies $\mathbb{C}^{\times}$-central extensions of the space $\mathcal{C}$, with the operations of sewing and disjoint union. The theorem says that all such holomorphic central extensions are essentially of the form $D e t^{\otimes n}$ where $n \in \mathbb{Z}$, and Det is the determinant line of Quillen [18]. Two details are missing in this description: first, for odd powers of Det, the $\mathbb{C}^{\times}$-central extensions are 'super' (or $\mathbb{Z} / 2$-graded, with appropriate signs). Second, the statement is only true modulo certain "trivial" central extensions which will be mentioned below. This paper contains two preliminary sections. In Section 2, we will define the relevant algebraic structures on the moduli spaces of surfaces $\mathcal{C}$, $\mathcal{C}_{\text {spin }}, \mathcal{C}_{\mathbb{Z} / 2}$, and $\mathbb{C}^{\times}$-central extensions. In Section 3, we shall review the basics of conformal field theory.

When extending the modularity formula to conformal field theories with Spin- and Sqrtstructure, the crucial step is to extend Mumford's theorem so as to classify holomorphic $\mathbb{C}^{\times}$-central extensions of $\mathcal{C}_{\text {spin }}$ and $\mathcal{C}_{\mathbb{Z} / 2}$. Up to equivalence, (i.e. modulo trivial $\mathbb{C}^{\times}$-central extensions), this is done in Section 4. It turns out that both groups of equivalence classes (in a suitable sense) of central extensions are isomorphic to $\mathbb{Z} \times \mathbb{Z} / 4 \mathbb{Z}$, and we identify the generators. In the case of $\mathcal{C}_{\text {spin }}$, the generators are the Quillen determinant, and the conformal anomaly of the CFT $\mathcal{F}\left(\Omega^{1 / 2}\right)$. In the case of $\mathcal{C}_{\mathbb{Z} / 2}$, the generators are the Quillen determinant, which is the conformal anomaly of $\mathcal{F}\left(\Omega^{0}\right)$, and a twisted version of the Quillen determinant, which, instead of functions (i.e. $\Omega^{0}$ ), uses sections of the complex line bundle associated with the $S q r t$-structure $L$.

Despite the classification of $\mathbb{Z} / 2$-graded $\mathbb{C}^{\times}$-central extensions of $\mathcal{C}_{\text {spin }}$ given in Section 4 , intuition suggests very strongly that with a suitable generalization of the axioms, the $\mathbb{C}^{\times}$-central extension of $\mathcal{C}_{\text {spin }}$ given by $\mathcal{F}\left(\Omega^{1 / 2}\right)$ should have a square root, i.e. a Pfaffian of the space of $1 / 2$-forms. While this Pfaffian is easy to define in certain cases (for example on the moduli space of Spin-surfaces with antiperiodic boundary components only), the axioms in the general case require the formalism of the super-Brauer group of $\mathbb{C}$, and were suggested to me by P. Deligne [4]. This is covered in Section 5.

"Trivial" $\mathbb{C}^{\times}$-central extensions are discussed in Section 6. These are certain holomorphic $\mathbb{C}^{\times}$-central extensions which are constant lines on the moduli spaces of curves of given genus, and depend only on the diffeomorphic type of the curve (with its Spin-structure). We classify in detail such trivial $\mathbb{C}^{\times}$-central extensions of $\mathcal{C}, \mathcal{C}_{\text {spin }}$.

In Section 7, we review some basic facts about automorphic functions of level 1 and 2, and in Section 8 we use them to obtain modularity formulas for $Z(\tau)$ in the case of Spin-CFT's, 
and $Z^{-}(\tau)$ in the case of Sqrt-CFT's. The groups of modular transformations occuring in these cases are subgroups of $S L_{2}(\mathbb{Z})$ of modular transformations of elliptic curves with distinguished point of order 2 . These are three subgroups $\Gamma_{1}, \Gamma_{2}, \Gamma_{3}$ of index 3 in $S L_{2}(\mathbb{Z})$, which contain the group $\Gamma(2)$ of order 2 modular transformations as an index 2 subgroup (one must pass to central extensions of the modular groups by $\mathbb{Z} / 2$ in the Spin case). The modularity formulas assert that after multiplying by $q^{-c / 24}$, the partition functions become essentially modular (of weight 0 ) with respect to the appropriate $\Gamma_{i}$, up to a character of $\Gamma_{i}$ which is determined by the conformal anomaly considered as an element of the group of $\mathbb{C}^{\times}$-central extensions of $\mathcal{C}_{\text {spin }}, \mathcal{C}_{\mathbb{Z} / 2}$.

\section{Moduli spaces of surfaces and their $\mathbb{C}^{\times}$-central extensions}

In this paper, the word surface always refers to a real 2-manifold or complex curve, i.e. not a complex surface. We will work with conformal surfaces $X$, by which we shall mean oriented smooth real 2-manifolds with boundary and a conformal structure. This is the same thing as a smooth oriented Riemann surface, where two Riemann metrics are considered equivalent if one is a scalar multiple of the other at each point. Further, we will assume that the boundary components of $X$ are parametrized by diffeomorphisms with $S^{1}$. There are two choices of orientation of such parametrization, according to which we shall distinguish the boundary components as incoming or outgoing. We label a boundary component as outgoing if the basis $\left(v, v_{\text {out }}\right)$ gives positive orientation where $v$ is the image of the tangent vector $(0,1)^{T}$ to $S^{1} \subset \mathbb{C}$ at the point $1 \in \mathbb{C}$, and $v_{\text {out }}$ is the normal vector to the boundary component of $X$, pointed outward. Thus, the boundary of the unit disk in $\mathbb{C}$ parametrized by $I d: S^{1} \rightarrow S^{1}$ is incoming.

For two conformal surfaces $X, Y$ we can obviously consider the conformal surface $X \amalg Y$. On the other hand, if we choose one incoming and one outgoing boundary component $c_{\text {in }}, c_{\text {out }}$ of a conformal surface $X$, we can define another conformal surface $\check{X}$ by gluing $c_{\text {in }}$ with $c_{\text {out }}$ by their parametrizations; if we choose a Riemann metric on $X$, the Riemann metrics on the two sides of the identified boundary components $c_{i n}, c_{o u t}$ may not be compatible. They can, however, be multiplied smoothly by scalars at each point in such a way that the resulting metric does give a well defined Riemann metric on $\check{X}$. Further, it can be shown that the resulting conformal structure does not depend on the choice of metric. We will work with the moduli space $\mathcal{C}$ of conformal surfaces. The connected components of $\mathcal{C}$ consisting of surfaces which do not have closed components can be viewed as $\infty$-dimensional complex manifolds [21]. The components consisting of closed surfaces are finite-dimensional, but can have automorphisms, and hence should be viewed as an analytic stack. Essentially, rather than just the space of isomorphism classes, we want to consider the Teichmüller space with the action of the modular group. We shall elaborate more on this below.

For now, let us consider the algebraic structure on $\mathcal{C}$. First, $\mathcal{C}$ is a category where the morphisms are isomorphisms of surfaces. Second, we have two operations on $\mathcal{C}$ : disjoint union $\amalg$ and sewing ? along a specified pair of incoming and outgoing boundary component. We then have the following requirements:

a symmetric monoidal structure on $\mathcal{C}$ with respect to the operation $\amalg$

$$
\text { an isomorphism } X \amalg \check{Y} \cong(X \check{\amalg} Y)
$$

an isomorphism between surfaces obtained by sewing two fixed pairs of boundary components, but with reversed order of sewing. 
These isomorphisms are required to give commutative coherence diagrams obtained whenever one expression in the operations $\amalg$ and ? can be converted to another using the isos (2.1)-(2.3) in two different ways.

It is tedious to write down explicitly all the coherence diagrams arising, but we shall give one example. Consider a surface $X \in \mathcal{C}$ with three distinguished pairs of boundary components, each containing one inbound and one outbound boundary component. Denoting by $\check{X}_{i, j, k}$, $\{i, j, k\}=\{1,2,3\}$, the surface obtained from $X$ by sewing the $i$ th, $j$ th and $k$ th pair in this order, then the isomorphisms (2.3) specify two compositions of isos

$$
\check{X}_{123} \cong \check{X}_{213} \cong \check{X}_{231}
$$

and

$$
\check{X}_{123} \cong \check{X}_{132} \cong \check{X}_{321} \cong \check{X}_{231} \text {. }
$$

The corresponding coherence diagram says that these two compositions are required to coincide.

To axiomatize the structure for which these axioms make sense precisely, note that for each conformal surface $X$ we have a pair

$$
\omega(X)=\left(X_{\text {in }}, X_{\text {out }}\right)
$$

of sets of inbound and outbound boundary components of $X$. The sewing operators give, for $a \in X_{\text {in }}, b \in X_{\text {out }}$, a surface

$$
\check{X}(a, b)
$$

where

$$
\check{X}(a, b)_{\text {in }}=X_{\text {in }}-\{a\}, \quad \check{X}(a, b)_{\text {out }}=X_{\text {out }}-\{b\} .
$$

A category Cat with such structure, i.e. assigning to each object $X$ a pair of sets $\omega(X)$ and a corresponding sewing operator as above, satisfying the axioms (2.1)-(2.3) and the respective coherence diagrams, will be called category with sewing. We shall further require that Cat be a groupoid.

We now define a $\left(\mathbb{Z} / 2\right.$-graded) $\mathbb{C}^{\times}$-central extension of a category with sewing Cat as a collection of $\mathbb{Z} / 2$-graded complex lines $D_{X}$ (i.e. pairs $\left(L, \varepsilon_{L}\right)$ where $L$ is a line and $\varepsilon_{L} \in \mathbb{Z} / 2$ ), indexed over objects $X \in \mathcal{C}$ and functorial there with respect to isomorphisms in Cat, together with natural isomorphisms

$$
\begin{aligned}
& D_{X \amalg Y} \cong D_{X} \otimes D_{Y}, \\
& D_{\check{X}} \cong D_{X} .
\end{aligned}
$$

The isomorphisms are coherent in the sense that diagrams of the following kind commute, where the arrows are the specified natural isomorphisms:

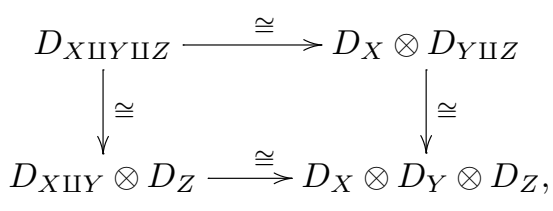



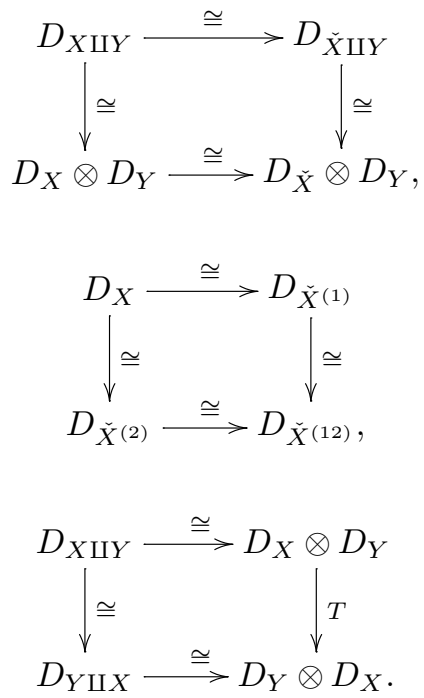

The penultimate diagram expresses commutativity of sewing isomorphism when we change the order of sewing. In the last diagram, the left column is functoriality, the right column is given by exchange of factors multiplied by $(-1)^{\varepsilon_{D_{X}} \varepsilon_{D_{Y}}}$.

These diagrams are important: it would be wrong to require that all isomorphisms between the lines $D_{X}$ obtained from (2.4) be compatible with all of the operations. For example, if $C a t=\mathcal{C}$ and $D_{+}$and $D_{-}$are copies of the unit disk in $\mathbb{C}$ with boundary parametrized by $z$, $z^{-1}$, respectively (thus, the boundary of $D_{+}$is outgoing and the boundary of $D_{-}$is incoming), and $A$ is any annulus, then by the Riemann mapping theorem, we obtain an isomorphism

$$
D_{D_{+}} \otimes D_{A} \otimes D_{D_{-}} \stackrel{\cong}{\longrightarrow} D_{\mathbb{P}^{1}}
$$

which gives as isomorphism

$$
D_{A} \cong D_{A^{\prime}}
$$

for any two annuli $A, A^{\prime}$. However, those isomorphisms cannot be compatible with gluing, since, as we shall see, $D$ is characterized by the $\mathbb{C}^{\times}$-central extension it specifies on the semigroup of annuli (and can be non-trivial - see [21], Chapter 5).

We would like to define a $\mathbb{C}^{\times}$-central extension of $\mathcal{C}$ holomorphic if for a holomorphic family of conformal surfaces $\left(X_{b}\right)_{b \in B}$ where $B$ is a complex manifold, $L\left(X_{b}\right)$ form holomorphic vector bundle on $B$.

To be completely precise about what 'holomorphic families of conformal surfaces' mean, actually, we must introduce the language of stacks $[6,13]$. We will consider the ground category $\mathcal{M}$ of smooth, but possibly infinite-dimensional (see [21,16]) complex manifolds with the analytic topology (i.e. coverings are coverings by systems of open sets). Over this category $\mathcal{M}$, a stack with sewing will then be defined as a symmetric monoidal stack $\mathcal{F}$ with operation $\amalg$, and also a map of stacks

$$
\omega: \mathcal{F} \rightarrow \mathcal{S}_{\text {in }} \times \mathcal{S}_{\text {out }}
$$

where the target is a product of two copies of the stack $\mathcal{S}$ whose sections over $B \in \operatorname{Obj}(\mathcal{M})$ are finite covering spaces of $B$. Further, for $X \in \operatorname{Obj}\left(\mathcal{F}_{B}\right)$ (the right hand side denoting objects over $B)$, if $\omega(X)=\left(S_{\text {in }}, S_{\text {out }}\right)$ and $a, b$ are sections of $S_{\text {in }}, S_{\text {out }}$ over $B$, then we require that 
we have a functorial

$$
\check{X}(a, b) \in \operatorname{Obj}(\mathcal{F})
$$

where

$$
\omega(\check{X}(a, b))=\left(S_{\text {in }}-\{a\}, S_{\text {out }}-\{b\}\right)
$$

(in the obvious sense). Stack versions of the axioms (2.1)-(2.3), and the corresponding coherences are also required.

To define a (holomorphic) $\mathbb{C}^{\times}$-central extension of a stack with sewing, we require that for each $B \in \operatorname{Obj} \mathcal{M}, X \in \operatorname{Obj}\left(\mathcal{F}_{B}\right), D_{X}$ be a (holomorphic) complex line bundle over $B$, with functoriality and all operations in the category of (holomorphic) line bundles.

The most fundamental example of stack with sewing is the moduli stack $\mathcal{C}$ of conformal surfaces with parametrized boundary components: the sections of $\mathcal{C}$ over $B \in \mathcal{M}$ are holomorphic families of surfaces over $B$, i.e. complex manifolds $M$ with a holomorphic projection $M \rightarrow B$ transverse to every point where the fiber is a surface (= complex curve), and a map $\partial M \rightarrow S^{1}$ which restricts to a boundary parametrization on each fiber (i.e. diffeomorphism on each boundary component of the fiber). Morphisms are holomorphic diffeomorphisms compatible with the projection to $B$ and boundary parametrization. Note that the category denoted above as $\mathcal{C}$ is the category of sections of the stack $\mathcal{C}$ over a point.

Another example $\mathcal{C}^{0}$ of stack with sewing has the same objects as $\mathcal{C}$, but morphisms are all isotopy classes of orientation preserving fibered diffeomorphisms (not necessarily holomorphic) compatible with boundary parametrization. The isotopy is required to be through maps of the same kind, i.e. fibered and preserving boundary parametrization.

Finally, we can define a stack with sewing $\mathcal{C}^{\text {top }}$ which has the same objects as $\mathcal{C}, \mathcal{C}^{0}$, and morphisms are equivalence classes of morphisms in $\mathcal{C}^{0}$ where two morphisms are defined to be equivalent if they induce the same maps on the sets of boundary components and connected components. We have obvious maps of stacks with sewing

$$
\mathcal{C} \rightarrow \mathcal{C}^{0} \rightarrow \mathcal{C}^{\text {top }}
$$

All the stacks we will use are analytic in the sense that they are equivalent to stacks of the form $[V / G]$ where $V$ is a smooth (possibly infinite-dimensional) complex manifold with properly discontinuous $G$-action. The sections of $[V / G]$ over a complex manifold $U$ are free $G$-equivariant manifolds $U^{\prime}$ with $G$-equivariant map $U^{\prime} \rightarrow V$ and an isomorphism $U^{\prime} / G \cong U$.

To explore this point further, we introduce some additional formalism. For categories with sewing $C_{1}, C_{2}$, a lax functor with sewing from $C_{1}$ to $C_{2}$ consists of

(1) a functor $F: C_{1} \rightarrow C_{2}$

(2) a natural isomorphism

$$
\lambda: F(X \amalg Y) \rightarrow F(X) \amalg F(Y)
$$

(3) a natural isomorphism

$$
\phi: \omega(X) \rightarrow \omega(F(X))
$$

(4) a natural isomorphism

$$
\mu: F(\check{X}(a, b)) \rightarrow F(\check{X})(\phi(a, b))
$$


for which the respective induced maps form commutative diagrams with the isos (2.1)-(2.3). For example, the commutative diagrams with (2.1) say that $F$ is a lax functor of symmetric monoidal categories. The commutative diagram with (2.3) is of the form

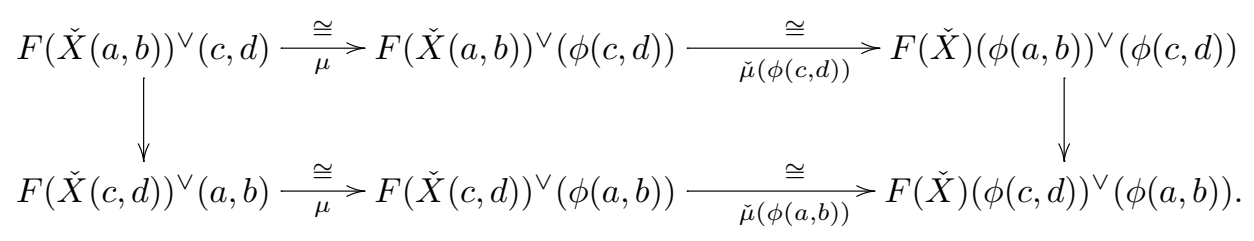

$\left(\right.$ Here $X^{\vee}:=\check{X}$.)

For two lax functors with sewing $F, G: C_{1} \rightarrow C_{2}$, an isomorphism with sewing is a natural isomorphism

$$
\eta: F \rightarrow G
$$

which commutes with the maps $\lambda, \phi, \mu$. In particular, for (3) this means the diagram

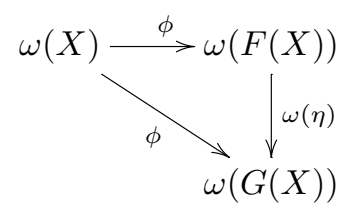

and for (4)

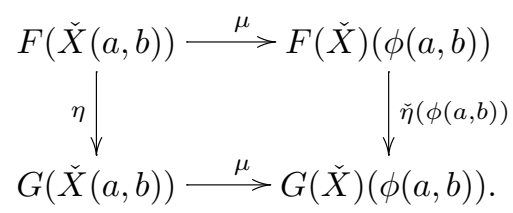

There are obvious versions of these definitions for stacks with sewing. An equivalence of categories with sewing $C_{1}, C_{2}$ consists of a pair of lax functors with sewing $F: C_{1} \rightarrow C_{2}$, $G: C_{2} \rightarrow C_{1}$ and isomorphisms with sewing $F G \cong I d, G F \cong I d$.

LEMMA 2.9. - A lax functor with sewing $F: C_{1} \rightarrow C_{2}$ between categories with sewing (resp. stacks with sewing) defines for every (holomorphic) $\mathbb{C}^{\times}$-central extension $D$ of $C_{2}$ a canonical (holomorphic) $\mathbb{C}^{\times}$-central extension $F^{*} D$ of $C_{1}$. An isomorphism with sewing $\eta: F \rightarrow G$ induces an isomorphism of (holomorphic) $\mathbb{C}^{\times}$-central extensions

$$
F^{*}(D) \cong G^{*}(D)
$$

Proof. - Define

$$
F^{*}(D)_{X}:=D_{F(X)}
$$

The axioms for lax functors with sewing give the structure needed. For an isomorphism with sewing

$$
\eta: F \rightarrow G
$$


the induced isomorphism on $\mathbb{C}^{\times}$-central extensions is

$$
F^{*}(D)_{X}=D_{F(X)} \underset{D_{\eta}}{\longrightarrow} D_{G(X)}=G^{*}(D)_{X}
$$

The required properties are easily verified.

In the case of compact conformal surfaces with parametrized boundary, recall that for surfaces which are of given topological type, there is a (infinite-dimensional, if the boundary is nonempty) contractible smooth manifold $T$ called the Teichmüller space on which there acts a modular group.

To define the Teichmüller space, select a surface $X_{0}$ with complex structure and parametrized boundary. The Teichmüller space (or fine moduli space) $T=T\left(X_{0}\right)$ can be defined as the space of isomorphism classes of data of the form

$$
\phi: X \rightarrow X_{0}
$$

where $\phi$ is an isotopy class of diffeomorphisms preserving orientation and boundary parametrization, and $X$ is a surface with complex structure and parametrized boundary.

Denote by $\operatorname{Diff}^{+}\left(X_{0}\right)$ the group of orientation preserving diffeomorphisms $X_{0} \rightarrow X_{0}$ respecting boundary parametrization, and denote by $\operatorname{Diff} 0_{0}^{+}\left(X_{0}\right)$ the subgroup of diffeomorphisms which are isotopic to the identity (through diffeomorphisms respecting boundary parametrization). Then the modular group (or mapping class group) $G=G\left(X_{0}\right)$ is defined as

$$
G=\operatorname{Diff}^{+}\left(X_{0}\right) / \operatorname{Diff}_{0}^{+}\left(X_{0}\right) .
$$

We have a short exact sequence of groups

$$
1 \rightarrow G_{0} \rightarrow G \rightarrow S \rightarrow 1
$$

where $G_{0}=G_{0}\left(X_{0}\right)$ is the subgroup of $G$ consisting of diffeomorphisms fixing boundary components and connected components, and $S=S\left(X_{0}\right)$ is the group of allowable permutations of boundary components.

The group $S$ is a semidirect product of the form

$$
S=S_{0} \rtimes S_{C}
$$

where $S_{C}=S_{C}\left(X_{0}\right)$ is the group of all permutations of connected components of $X_{0}$ preserving isomorphism type of connected components, and $S_{0}$ is the product over connected components $C$ of the groups of all permutations of boundary components of $X_{0}$ contained in $C$, which preserve orientation (i.e. which send inbound boundary components to inbound and outbound boundary components to outbound).

Now recall that there is an equivalence of stacks

$$
\mathcal{C} \stackrel{\cong}{\longrightarrow} \coprod_{X_{0}}\left[T\left(X_{0}\right) / G\left(X_{0}\right)\right]
$$

where the disjoint union is over representatives of boundary parametrization preserving diffeomorphism of conformal surfaces. Similarly, we have equivalences of stacks

$$
\mathcal{C}^{0} \stackrel{\cong}{\longrightarrow} \coprod_{X_{0}}\left[* / G\left(X_{0}\right)\right]
$$




$$
\mathcal{C}^{\text {top }} \stackrel{\cong}{\longrightarrow} \coprod_{X_{0}}\left[* / S\left(X_{0}\right)\right]
$$

Note that we can use these equivalences to induce structures of stacks with sewing on the right hand sides of (2.12)-(2.14), so that (2.12)-(2.14) then become equivalences of stacks with sewing. However, note that the equivalences of stacks (2.12)-(2.14) are not canonical: there is merely a distinguished class of equivalences any two of which are naturally isomorphic. Thus, the induced structures of stacks with sewing on the right hand sides of (2.12)-(2.14) are not canonical, although any two such structures are equivalent.

Note that this discussion is an elaboration of a precisely analogous discussion for symmetric monoidal categories.

We will see that $\mathbb{C}^{\times}$-central extensions of $\mathcal{C}^{0}$ correspond exactly to $\mathbb{C}^{\times}$-central extensions of $\mathcal{C}$ of central charge 0 (see Lemma 4.3). Also, $\mathbb{C}^{\times}$-central extensions of $\mathcal{C}^{\text {top }}$ correspond precisely to $\mathbb{C}^{\times}$-central extensions of $\mathcal{C}$ of central charge and rotation number 0 . We shall call such central extensions trivial. We shall see that in this case, they are the same thing as $\mathbb{C}^{\times}$-central extensions of $\mathcal{C}^{0}$, but that will be no longer true if we replace $\mathcal{C}$ by stacks of surfaces with additional structure, such as Spin. We shall return to this later.

An example of a holomorphic $\mathbb{C}^{\times}$-central extension of $\mathcal{C}$ is the determinant line Det. The definition of Det is explained in [21] (and also earlier in Quillen [18]). Another account is given in [14]. The definition we give here is dual to [14], to get formula (2.19) below. If $X$ has no closed components, we can define $\operatorname{Det}(X)$ as the dual of the determinant line in the Quillen sense of the Fredholm operator

$$
\pi_{X}: \operatorname{Hol}(X) \rightarrow \Omega_{+}^{0}(\partial X) .
$$

(Alternately, and perhaps preferably, we may think of (2.15) as a complex.) The right hand side means the negative subspace of $\Omega^{0}(\partial X)$. More precisely, instead of $\partial X$, we should use the notation $\coprod S^{1}$, as, using the parametrization, we are thinking of the boundary as a disjoint union of copies of the unit circle. A polarization of a Hilbert space $H$ is an explicit direct sum decomposition of $H$ into two subspaces:

$$
H=H_{+} \oplus H_{-} .
$$

In the standard polarization of $\Omega^{0}\left(S^{1}\right), \Omega_{+}^{0}\left(S^{1}\right)$ is spanned (in the topological sense) by $z^{n}$, $n \geqslant 0$ and $\Omega_{-}^{0}\left(S^{1}\right)$ is spanned (in the topological sense) by $z^{-n}, n>0$. This is the polarization we use in (2.15) on the incoming components of $\partial X$, while we use the opposite polarization on the outgoing components. The map (2.15) is given by restriction (via the parametrizing map), composed with projection to the negative subspace, multiplied by -1 on the incoming boundary components.

The Quillen determinant line $\operatorname{Det}_{F}$ of a Fredholm operator $F$ is in degree

$$
\varepsilon_{\text {Det }_{F}}=\operatorname{index}(F) \bmod 2 .
$$

For Fredholm operators $F$ of index 0, $\operatorname{Det}_{F}$ is defined as follows (see [16]): Suppose first that $F$ has index 0 . Let $F=J+K$ where $J$ is invertible, $K$ is of trace class. Let

$$
\operatorname{Det}_{F}=\mathbb{C}\{J\} .
$$

This construction is canonical, for if $F=J^{\prime}+K^{\prime}$, then

$$
J^{\prime}=J+\left(K-K^{\prime}\right)=J\left(I+J^{-1}\left(K-K^{\prime}\right)\right)
$$


where $I+J^{-1}\left(K-K^{\prime}\right)$ is of determinant class, so we have an isomorphism

$$
\mathbb{C}\left\{J^{\prime}\right\} \cong \mathbb{C}\{J\}
$$

by

$$
J^{\prime} \mapsto \operatorname{det}\left(I+J^{-1}\left(K-K^{\prime}\right)\right) J
$$

Further, [16], one defines

$$
\operatorname{det}(F) \in \operatorname{Det}(F)
$$

by $\operatorname{det}(Q J)=(\operatorname{det}(Q)) \cdot J$ if $J$ is invertible and $Q$ is of determinant class. From this point of view, (Det, det) generalizes the determinant of operators of determinant class: we have $\operatorname{det}(Q)=\operatorname{det}(Q) \cdot I d$.

To define the Quillen determinant for Fredholm operators of arbitrary index, first note that the above discussion in index 0 gives a canonical isomorphism

$$
\operatorname{Det}(F) \cong(\operatorname{Det}(\operatorname{Ker}(F)))^{-1} \otimes(\operatorname{Det}(\operatorname{Coker}(F))) .
$$

An explanation of the fact that this formula is the inverse of what is suggested by index and cohomology ultimately comes from the finite-dimensional case: for a linear map

$$
F: U \rightarrow V
$$

between vector spaces of equal finite dimension $n$, we are looking for a canonical line $\operatorname{Det}(F)$ which is canonically isomorphic for different choices of $F$ with the same $U, V$ and has a canonical element $\operatorname{det}(F) \in \operatorname{Det}(F)$. We see that one can take

$$
\operatorname{Det}(F)=\operatorname{Det}(\operatorname{Coker}(F)) \otimes \operatorname{Det}(\operatorname{Ker}(F))^{-1},
$$

which is canonically isomorhpic to

$$
\operatorname{Det}(V) \otimes \operatorname{Det}(U)^{-1} \cong \Lambda^{n}(V) \otimes \Lambda^{n}(U)^{*}
$$

and has canonical element corresponding to the map induced by $F$ on top exterior powers.

Granted that, we can use (2.16) to define the determinant line of a Fredholm operator of arbitrary index, keeping in mind that the determinant line of a finite-dimensional space $V$ is in degree $\operatorname{dim}(V) \bmod 2$. The above discussion can then be interpreted as a construction of a canonical isomorphism between the determinant lines of two operators whose difference is trace class (i.e. a kind of "continuity result").

To prove (2.16), choose a splitting $\operatorname{Ker}(F) \oplus U=H$, and a lift of the map $H \rightarrow \operatorname{Coker}(F)$, which gives a splitting $\operatorname{Coker}(F) \oplus V=H$. Then we can choose the invertible operator $J$ as $J=J_{1} \oplus J_{2}$ where $J_{1}: \operatorname{Ker}(F) \stackrel{\cong}{\longrightarrow} \operatorname{Coker}(F), J_{2}: U \stackrel{\cong}{\longrightarrow} V$, and moreover $J_{2}$ coincides with a restriction of $F$. Then $J$ defines an element

$$
\operatorname{det}\left(J_{1}\right) \in(\operatorname{Det}(\operatorname{Ker}(F)))^{-1} \otimes(\operatorname{Det}(\operatorname{Coker}(F))) .
$$

Define the isomorphism (2.16) by sending $J \in \operatorname{Det}(F)$ to $\operatorname{Det}\left(J_{1}\right)$. We see easily that for another $J$ with these properties, (2.17) transforms according to the same rule as the generator of $\operatorname{Det}(F)$

$4^{\mathrm{e}}$ SÉRIE - TOME $36-2003-\mathrm{N}^{\circ} 1$ 
as defined above, so the isomorphism is well defined once we choose a splitting. But note that $\operatorname{Det}\left(J_{1}\right)$ does not depend on the splitting, since a change of splitting operator has determinant 1 .

The definition (2.15) does not work for closed surfaces. While the formula (2.15) makes sense technically, it gives just $\mathbb{C}$ for closed surfaces, which is the wrong answer (in the sense that the second gluing formula (2.4) would be false). The reason is that in the proof of the gluing formula ([21], proof of Proposition 6.4), one uses a diagram of the form

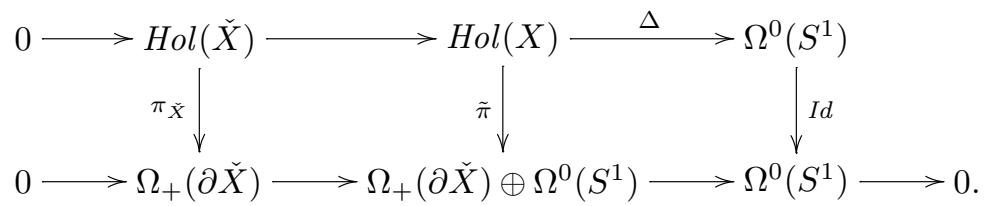

Here $\Delta$ is the sum of the two restrictions to the boundary components which are being glued, with sign reversed on the incoming component. The map $\tilde{\pi}$ is determined by the diagram, but it is easily seen that it differs from $\pi_{X}$ by an operator of trace class, and hence does not affect the determinant line. (Concretely, $\tilde{\pi}$ is just the restriction to the boundary, with sign reversed on incoming boundary components, while $\pi_{X}$ is the same map composed with projection to the positive subspace in the polarization.) This is essentially the proof of (2.4), if we know that $\Delta$ has dense image. This follows if $\check{X}$ has no closed components (because then it is a Stein manifold). However, $\Delta$ may not have dense image if $\check{X}$ is closed. One way to treat this is simply to define for $X$ closed $\operatorname{Det} X$ as the determinant line of the surface $X^{\prime}$ obtained by cutting out the holomorphic image of the standard disk (with boundary oriented as incoming with respect to $X^{\prime}$ ). This is in fact forced by (2.4). The definition, however requires showing that $\operatorname{Det}\left(X^{\prime}\right)$ does not depend on the choice of the disk.

The other way (which we will use) is to define, for a closed surface $Y$,

$$
\operatorname{Det}(Y)=\operatorname{Det}\left(H^{0}(Y, \underline{H o l})\right) \otimes \operatorname{Det}\left(H^{1}(Y, \underline{H o l})\right)^{-1}
$$

where on the right hand side, Det denotes the top exterior power. This is compatible with gluing since, for $Y=\check{X}$, the homology of the complex

$$
\operatorname{Hol}(X) \stackrel{\Delta}{\longrightarrow} \Omega^{0}\left(S^{1}\right)
$$

in $(2.18)$ is actually $H^{*}(\check{X}, \underline{H o l})$.

Note that (2.19) and (2.16) explain why for a surface $X$ with boundary, $\operatorname{Det}(X)$ is defined to be the dual of (2.15).

We will be interested in classifying holomorphic $\mathbb{C}^{\times}$-central extensions of $\mathcal{C}$. To state such classification correctly, note that we can readily replace Det by another central extension $D e t^{\prime}$ where

$$
\begin{aligned}
& \operatorname{Det}_{X} \cong \operatorname{Det}_{X}^{\prime}, \\
& \varepsilon_{\text {Det }_{X}}=\varepsilon_{\text {Det }_{X}^{\prime}}+d_{X}
\end{aligned}
$$

where $d_{X}$ is the number of boundary components of $X$. To define such $D e t^{\prime}$, it suffices to suitably alter the polarization of $\Omega^{0}\left(S^{1}\right)$ (and hence $\Omega^{0}(\partial X)$ in (2.15)). For example, we can change $\Omega_{+}^{0}\left(S^{1}\right)$ to $\left\langle z^{n} \mid n>0\right\rangle$.

It is easy to see that the holomorphic $\mathbb{C}^{\times}$-central extension

$$
(D e t)^{-1} \otimes \operatorname{Det}^{\prime}
$$


of $\mathcal{C}$ then defines trivial bundles over the stacks $\left[T / G_{0}\right]$ for connected components $[T / G]$ of $\mathcal{C}$ (see (2.11)). This means that (2.20) defines a bundle on each $[* / S]$, and hence a $\mathbb{C}^{\times}$-central extension of $\mathcal{C}^{t o p}$. Recall that we called such central extension trivial. Two central extensions $D, D^{\prime}$ of $\mathcal{C}$ will be called equivalent if $D \otimes\left(D^{\prime}\right)^{-1}$ is trivial. A classification of trivial central extensions will be given in the beginning of Section 4 .

THEOREM 2.21 (Mumford, see [21], Section 5). - The group of equivalence classes (with respect to the equivalence just defined) of holomorphic $\mathbb{C}^{\times}$-central extensions of $\mathcal{C}$ is isomorphic to $\mathbb{Z}$, with generator Det.

In Section 4, we will prove this theorem, and some generalizations, related to additional structure.

The first type of such structure is Spin-structure. Following Segal [21], a Spin-structure on a conformal surface $X$ is a holomorphic line bundle $L$ together with an isomorphism between $L \otimes L$ and the tangent bundle of $X$. On the other hand, for a real 1-manifold $\ell$ (i.e. a disjoint union on circles), a Spin-structure on $\ell$ is a real line bundle $P$ with an explicit isomorphism $P \otimes_{\mathbb{R}} P$ with the tangent bundle of $\ell$. An isomorphism of Spin-structures $L, L^{\prime}$ consists of an isomorphism

$$
\phi: L \stackrel{\cong}{\longrightarrow} L^{\prime}
$$

such that the diagram

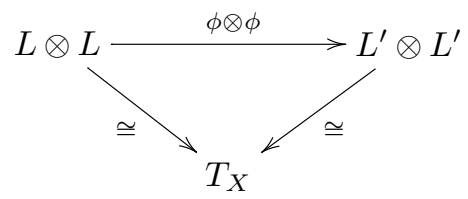

commutes where the diagonal arrows are from the definition of Spin-structure, and $T_{X}$ is the tangent bundle. There are, of course, two Spin-structures on $S^{1}$ : trivial and Möbius. As pointed out in [21], a Spin-structure on $X$ induces a Spin-structure on $\partial X$ : take the vectors in $L$ which are square roots of outgoing (resp. incoming) normal vectors at the boundary, depending on whether the boundary component is outgoing or incoming. We now define the space of holomorphic $k / 2$-forms $\Omega^{k / 2}(X)=\Omega_{H o l}^{k / 2}(X)$ as the space of sections of $L^{\otimes-k}$. (The reader should be careful not to confuse this with the Dolbeaux notation, which refers to sections of exterior, rather than symmetric, powers.) Of course, for $2 \mid k$, these spaces are defined independently of Spin-structure. We would like to remind the reader that while there may in many cases be no difference between the different powers of $L$ (for example, they may all be trivial), we are however also interested in maps of forms induced by holomorphic maps $f: X \rightarrow Y$ of conformal surfaces (not to mention parametrizations of the boundary), and those do depend on $k$ : there is, of course, the map $D f: L_{X}^{\otimes 2} \rightarrow L_{Y}^{\otimes 2}$, whose tensor powers induce maps on $\Omega^{k}, k \in \mathbb{Z}$. To obtain a map $L_{X} \rightarrow L_{Y}$, however, one must in addition to $f$ choose a square root of the map $D f$ (if one exists). We will refer to this as a choice of spin. A choice of spin of a map $f$ determines map on $\Omega^{k / 2}$ for all $k \in \mathbb{Z}$. Similar comments apply also to Spin-structures on real 1-manifolds. Regarding the boundary, note that a section of any bundle on $X$ (for example the Spin-structure) of course canonically restricts to the restriction of the bundle on the boundary (i.e. for example the complexification of the Spin-structure on the boundary). Therefore, this does not depend on the choice of $k$, just on the isomorphism class of the (line) bundles involved. However, we also parametrize the boundary components, and we see that it is critical, along with such parametrization, to make a choice of its spin. Then the requisite restriction of $k / 2$-forms to $\amalg S^{1}$ is equal to the canonical restriction to the boundary, composed with the map induced 
by the parametrization, with the chosen spin (if 2 does not divide $k$ ). The resulting map depends on $k$, even if the corresponding tensor powers of $L$ are isomorphic for different values of $k$.

An important example is our choice of Spin-structure on $A_{q}, q=\mathrm{e}^{2 \pi i \tau}$. Any holomorphic line bundle on the standard annulus

$$
A_{q}=\{z \in \mathbb{C}|| q \mid<\|z\|<1\}
$$

(with boundary components parametrized by $z$ and $q$, respectively) is trivial, so we may as well assume that $L=1$. There is, however, a choice of isomorphism $L^{\otimes 2} \cong T A_{q}$ : there are two essential such choices (all others lead to isomorphic data), namely the isomorphism 1 (the periodic Spin structure) and $z$ (the antiperiodic Spin structure). Note that by the above comments, this already determines Spin-structure on the boundary, which is trivial (periodic) in the former case, and Möbius (antiperiodic) in the latter. We shall denote these standard annuli by $A_{q, P}, A_{q, A}$.

We can consider the moduli space $\mathcal{C}_{\text {spin }}$ of all conformal subspaces with Spin-structure. Regarding the boundary, we shall make a fixed choice of two standard copies $S_{A}^{1}, S_{P}^{1}$ of $S^{1}$, with 'standard' (fixed) representatives of the two isomorphism classes of Spin-structures (antiperiodic and periodic). For example, let $S_{A}^{1}$ and $S_{P}^{1}$ be the inbound boundary components of the antiperiodic and periodic standard annuli $A_{q, A}, A_{q, P}$ (this does not depend on the choice of $q$ ). An element of $\mathcal{C}_{\text {spin }}$ then consists of a conformal surface with Spin-structure $X$, and parametrizations of the boundary components of $X$ (which have induced Spin-structures) by specified diffeomorphisms with $S_{A}^{1}$ or $S_{P}^{1}$, with chosen spin. By an isomorphism of surfaces with Spin-structure we shall mean a holomorphic diffeomorphism with spin which commutes with the boundary parametrizations, preserving also their spin. Therefore, we do not allow diffeomorphisms which induce spin reversal on $S_{A}^{1}, S_{P}^{1}$. For example, spin reversal of a Spinsurface with non-empty boundary will not be considered an automorphism.

This category $\mathcal{C}_{\text {spin }}$ enjoys the operations of sewing and, obviously, disjoint union, also. By definition, the sewing operation is completely determined by choice of the boundary components to be sewn: the spin of the sewing map will be chosen as identity on the standard $S_{A}^{1}$ or $S_{P}^{1}$.

However, unfortunately, $\mathcal{C}_{\text {spin }}$ is not a stack with sewing in the sense defined above because of the different types of boundary components. We need a generalization.

Define, for a small groupoid $K$, a stack $\mathcal{S}^{K}$ as follows: Sections over $B \in \mathcal{M}$ are functors

$$
F: K \rightarrow \text { (covering spaces of } B \text {, deck transformations) }
$$

(by deck transformations we mean isos commuting with projection) which satisfy

$$
\begin{aligned}
k \neq \ell \in \operatorname{Obj}(K) & \Rightarrow F(k) \cap F(\ell)=\emptyset, \\
f \in \operatorname{Mor}(K) \text { and } f \neq I d & \Rightarrow F(f) \text { has no fixed points. }
\end{aligned}
$$

Note that $\mathcal{S}^{K}$ enjoys a symmetric monoidal structure $\amalg$ defined in the obvious way.

Define a stack $\mathcal{F}$ with $K$-sewing as the following set of data:

(1) A symmetric monoidal structure on $\mathcal{F}$ with operation denoted by $\amalg$.

(2) A map of stacks

$$
\omega: \mathcal{F} \rightarrow \mathcal{S}_{\text {in }}^{K} \times \mathcal{S}_{\text {out }}^{K}
$$

where the target is a product of two copies of $\mathcal{S}^{K}$. 
(3) To a data $X \in \operatorname{Obj}\left(\mathcal{F}_{B}\right), \phi(X)=\left(F_{1}, F_{2}\right)$ and isos $F_{1} \cong F \amalg G_{1}, F_{2} \cong F \amalg G_{2}$ in $\mathcal{S}^{K}$, an assigned (functorial) object

$$
\check{X}, \quad \omega(\check{X})=\left(G_{1}, G_{2}\right) .
$$

(4) Isomorphisms analogous to (2.1)-(2.3), and coherences, analogous to the concept of stack with sewing.

A $(\mathbb{Z} / 2$-graded $) \mathbb{C}^{\times}$-central extension of a stack with $K$-sewing is defined analogously as for stacks with sewing.

Define the category 2 to be discrete with objects $\{A, P\}$. Then $\mathcal{C}_{\text {spin }}$ is a stack with 2 -sewing where for a Spin-surface $X$,

$$
\phi(X)=\left(F_{1}, F_{2}\right)
$$

where $F_{1}$ (resp. $F_{2}$ ) maps $A, P$ to the sets of all inbound (resp. outbound) boundary components of $X$ of the given Spin-structure.

This extends to holomorphic families of surfaces: The sections of $\mathcal{C}_{\text {spin }}$ over $B \in \operatorname{Obj}(\mathcal{M})$ are families of surfaces $X \rightarrow B$ with a map $\partial X \rightarrow S_{A}^{1} \amalg S_{P}^{1}$ with a holomorphic line bundle $L$ on $X$ and an iso $L \otimes L \cong T X_{\text {vert }}$ (the line bundle consisting of vectors tangent to the fiber). Then $L$ specifies a real line bundle $L^{\prime}$ on the boundary and an iso $L^{\prime} \otimes L^{\prime} \cong T \partial X_{\text {vert }}$, as above. We require, as a part of the structure, a map $L^{\prime} \rightarrow L_{S_{A}^{1} \amalg S_{P}^{1}}$ which induces spin on the boundary parametrizations of fibers.

Next, note that the definition of spin of course extends to non-holomorphic oriented diffeomorphisms of surfaces, because the inclusion of structure groups $\mathbb{C}^{\times} \subset G L_{2}^{+}$( $\mathbb{R}$ ) (the target is the group of real $2 \times 2$ matrices with positive determinant) is a homotopy equivalence. The same is true for the stabilizer subgroups of the positive ray of an oriented boundary component (they are contractible), and hence we can define, for two elements $X, Y$ of $\mathcal{C}_{\text {spin }}$ (nonholomorphic) Spin-diffeomorphisms $X \rightarrow Y$ inducing identity spin on boundary components. We shall call such maps 'Spin-diffeomorphisms compatible with boundary parametrization and its spin'.

Now define $\mathcal{C}_{\text {spin }}^{0}$ as the stack whose sections over $B$ are the same as the sections of $\mathcal{C}_{\text {spin }}$, but morphisms are isotopy classes of Spin-diffeomorphisms compatible boundary parametrization and its spin. The isotopies are through maps of the same kind. Then $\mathcal{C}_{\text {spin }}^{0}$ is also a stack with 2-sewing.

We also define $\mathcal{C}_{\text {spin }}^{\text {top }}$ to have the same objects as $\mathcal{C}_{\text {spin }}^{0}$ and morphisms equivalence classes of morphisms in $\mathcal{C}_{\text {spin }}^{0}$ under the equivalence relation where two morphisms are equivalent if and only if they induce the same maps on boundary components and connected components. Again, we have canonical maps of stacks with 2 -sewing

$$
\mathcal{C}_{\text {spin }} \rightarrow \mathcal{C}_{\text {spin }}^{0} \rightarrow \mathcal{C}_{\text {spin }}^{\text {top }}
$$

Again, a $\left(\mathbb{Z} / 2\right.$-graded) $\mathbb{C}^{\times}$-central extension of $\mathcal{C}_{\text {spin }}$ which is a pullback of a $\mathbb{C}^{\times}$-central extension of $\mathcal{C}_{\text {spin }}^{\text {top }}$ will be called trivial, and two $\mathbb{C}^{\times}$-central extensions whose ratio is trivial will be called equivalent.

Now for $X_{0} \in \mathcal{C}_{\text {spin }}$, we can define the Teichmüller space as the space of isomorphic classes of data of the form

$$
\phi: X \rightarrow X_{0}
$$

where $\phi$ is an isotopy class (through maps of the same kind) of Spin-diffeomorphisms compatible with boundary parametrization. However, note that the Spin-structure on $X$ 
(including spin of boundary parametrizations) is then induced by $\phi$, and hence this Teichmüller space is canonically isomorphic to the already familiar Teichmüller space $T\left(X_{0}\right)$. The Spin-mapping class group, however, is

$$
G^{\text {spin }}=G^{\text {spin }}\left(X_{0}\right)=\operatorname{Diff}_{S}\left(X_{0}\right) / \operatorname{Diff}_{0}^{+}\left(S^{0}\right)
$$

where $\operatorname{Diff}_{S}\left(X_{0}\right)$ is the group of Spin-diffeomorphisms of $X_{0}$ compatible with boundary parametrization.

Similarly as in the case of $\mathcal{C}$, we have a short exact sequence of groups

$$
1 \rightarrow G_{0}^{\text {spin }} \rightarrow G^{\text {spin }} \rightarrow S^{\text {spin }} \rightarrow 1
$$

where $G_{0}^{\text {spin }}$ is the subgroup fixing boundary components and connected components of $X_{0}$, $S^{\text {spin }}=S^{\text {spin }}\left(X_{0}\right)$ is the group of allowable permutations of boundary components. The group $S^{\text {spin }}$ is again of the form

$$
S_{0}^{s p i n} \rtimes S_{C}^{s p i n}
$$

where $S_{C}^{\text {spin }}$ is the group of all permutations of connected components of $X_{0}$ preserving Diff $S_{S}$-type, and $S_{0}^{\text {spin }}$ is the product over the set of connected components of the allowable permutations of boundary components in the individual connected components.

LEMMA 2.24. - If $X_{0}$ is connected, then $S_{0}^{\text {spin }}$ is the group of all permutations preserving type of boudary components (i.e. inbound vs. outbound, periodic vs. antiperiodic), and such that the induced permutation on the set of periodic boundary components is even.

Proof. - We might as well assume that $X_{0}$ has outbound periodic boundary components only. First, observe that for a periodic annulus $B$ with two outbound boundary components switching the boundary components, a Spin-diffeomorphism of $B$ switching the boundary components preserves the spin of one, and reverses the other. Taking connected sum, we obtain a Spin diffeomorphism of $X_{0}$ switching two boundary components, while reversing the spin of one of them. Composing such maps, we can obtain a Spin-diffeomorphism of $X_{0}$ compatible with boundary parametrization which induces any even permutation on the boundary components, as claimed.

Thus, it remains to show that no Spin-diffeomorphism of $X_{0}$ compatible with boundary parametrization can induce an odd permutation of the (periodic) boundary components. If this was possible, from what we just showed it would follow that there exists a Spindiffeomorphism of $X_{0}$ which preserves the boundary components, and reverses spin on precisely one boundary component. Reversing orientation of boundary components and sewing, we may further assume that there are only two (periodic) boundary components with opposite orientation. A contradiction then follows from the following

Lemma 2.25. - Suppose $X$ is a closed Spin-surface. Let $X^{\prime}$ be obtained from $X$ by cutting along a periodic simple curve, and sewing back with opposite spin. Then the surfaces $X, X^{\prime}$ are not Spin-diffeomorphic.

Proof. - By Segal [21], a Spin-structure on a closed oriented surface $X$ corresponds to a quadratic form associated with the canonical symmetric bilinear form on $H^{1}(X, \mathbb{Z} / 2)$. By definition, the quadratic forms corresponding to $X, X^{\prime}$ have different Kervaire invariants [2]. 
Note that we have again equivalences of stacks

$$
\mathcal{C}_{\text {spin }} \stackrel{\cong}{\longrightarrow} \coprod_{X_{0}}\left[T\left(X_{0}\right) / G^{\text {spin }}\left(X_{0}\right)\right]
$$

where $X_{0}$ runs through representatives of equivalence classes of Spin-surfaces under Spindiffeomorphisms compatible with boundary parametrization and its spin. Then also

$$
\begin{gathered}
\mathcal{C}_{\text {spin }}^{0} \stackrel{\cong}{\longrightarrow} \coprod_{X_{0}}\left[* / G^{\text {spin }}\left(X_{0}\right)\right], \\
\mathcal{C}_{\text {spin }} \stackrel{\cong}{\longrightarrow} \coprod_{X_{0}}\left[* / S^{\operatorname{spin}}\left(X_{0}\right)\right],
\end{gathered}
$$

and similar discussion applies as in the case of $\mathcal{C}$.

We will also need to consider the structure we obtain when, instead of Spin-structure, we specify a square root of the trivial bundle on $X \in \mathcal{C}$, i.e. a complex holomorphic line bundle $L$ together with an isomorphism

$$
L \otimes L \cong 1_{X}
$$

We shall call this a square root structure (Sqrt-structure). It is equivalent to specifying a principal $\mathbb{Z} / 2$-bundle on $X$, which, of course, restricts to a principal $\mathbb{Z} / 2$-bundle on $\partial X$. One fixes, once again, representatives of the two non-isomorphic $\mathbb{Z} / 2$-bundles on $S^{1}$ (periodic and antiperiodic), and specifies, along with a parametrization of the boundary components of $\partial X$, the accompanying map of $\mathbb{Z} / 2$-modules. In Segal [21], the moduli space of conformal surfaces with $S q r t$-structure is denoted by $\mathcal{C}_{\mathbb{Z} / 2}$ (in fact, an analogous structure is considered for any finite group $G$ ). Once again, we can define $\mathbb{C}^{\times}$-central extensions of $\mathcal{C}_{\mathbb{Z} / 2}$, which will be classified in the next section. Once again, $\mathcal{C}_{\mathbb{Z} / 2}$ contains $\mathcal{A}_{A}$ and $\mathcal{A}_{P}$. However, notice that in $\mathcal{C}_{\mathbb{Z} / 2}$, the boundary of the standard disk $D$ is periodic, while in $\mathcal{C}_{\text {spin }}$ it is antiperiodic.

Again, we find that $\mathcal{C}_{\mathbb{Z} / 2}$ has the structure of stack with 2-sewing, and analogously as in the Spin-case, we have maps of stacks with 2-sewing

$$
\mathcal{C}_{\mathbb{Z} / 2} \rightarrow \mathcal{C}_{\mathbb{Z} / 2}^{0} \rightarrow \mathcal{C}_{\mathbb{Z} / 2}^{\text {top }}
$$

Remark. - There are coarser variants $\mathcal{C}_{\text {spin }}^{c}, \mathcal{C}_{\mathbb{Z} / 2}^{c}$ of the moduli spaces $\mathcal{C}_{\text {spin }}, \mathcal{C}_{\mathbb{Z} / 2}$, obtained by including in the mapping class group diffeomorphisms inducing non-trivial automorphisms of the structure on the boundary components.

To this end, let $2_{C}$ be the groupoid with two objects $A, P$ each of which has automorphism group isomorphic to $\mathbb{Z} / 2$. Then $\mathcal{C}_{\text {spin }}^{c}$ is defined as the stack whose sections over $B \in \operatorname{Obj}(\mathcal{M})$ are holomorphic families $X \rightarrow B$ of conformal surfaces with boundary parametrization $\partial X \rightarrow S_{A}^{1} \amalg S_{P}^{1}$ together with an isomorphism

$$
L \otimes L \stackrel{\cong}{\longrightarrow} T(X)_{\text {vert }}
$$

We also require that the target of the boundary parametrization of a boundary component of a fiber match its Spin-structure, but we do not specify the spin of the boundary parametrization.

Then $\mathcal{C}_{\text {spin }}^{c}$ is naturally a stack with $2_{C}$-sewing. The map

$$
\mathcal{C}_{\text {spin }}^{c} \rightarrow \mathcal{S}_{2_{C}}^{\text {in }} \times \mathcal{S}_{2_{C}}^{\text {out }}
$$


assigns to a family $M \rightarrow B$ a pair of functors $\left(F_{1}, F_{2}\right)$ where $F_{1}(A), F_{1}(P), F_{2}(A), F_{2}(P)$ are covering spaces of $B$ whose fibers over $x \in B$ consist of possible maps

$$
\begin{gathered}
\left\{L_{C} \rightarrow L_{S_{A}^{1}} \mid C \text { is an inbound antiperiodic boundary component of } X_{x}\right\}, \\
\left\{L_{C} \rightarrow L_{S_{P}^{1}} \mid C \text { is an inbound periodic boundary component of } X_{x}\right\}, \\
\left\{L_{C} \rightarrow L_{S_{A}^{1}} \mid C \text { is an outbound antiperiodic boundary component of } X_{x}\right\}, \\
\left\{L_{C} \rightarrow L_{S_{P}^{1}} \mid C \text { is an outbound periodic boundary component of } X_{x}\right\},
\end{gathered}
$$

which define spin on the corresponding boundary component parametrization (i.e. are square roots of the differential of the boundary component parametrization $-L_{C}$ is the Spin-structure on the boundary component $C$ induced from the Spin-structure of $X_{x}$ ). The $\mathbb{Z} / 2$-actions are by reversal of sign.

$\mathcal{C}_{\text {spin }}^{c, 0}$ is defined to have the same objects as $\mathcal{C}_{\text {spin }}^{c}$, where morphisms are isotopy classes (through maps of the same kind) of diffeomorphisms with spin which preserve boundary parametrization, but not necessarily its spin. $\mathcal{C}_{\text {spin }}^{c, t o p}$ is defined the same way except that two morphisms are identified if they induce the same map on boundary components and connected components, and also the same spin on boundary component parametrization. We have canonical maps of stacks with $2_{C}$-sewing

$$
\mathcal{C}_{\text {spin }}^{c} \rightarrow \mathcal{C}_{\text {spin }}^{c, 0} \rightarrow \mathcal{C}_{\text {spin }}^{c, \text { top }}
$$

Now let $\operatorname{Diff}_{C}\left(X_{0}\right)$ denote the group of $C$-diffeomorphisms of $X_{0}$, by which we mean Spin-diffeomorphisms of which preserve, as diffeomorphisms, the parametrizations of boundary components, but not necessarily the spin of these parametrizations. Define the mapping class group

$$
G_{C}=G_{C}\left(X_{0}\right)=\operatorname{Diff}_{C}\left(X_{0}\right) / \operatorname{Diff}_{0}^{+}\left(X_{0}\right) .
$$

Then we have equivalences of stacks

$$
\begin{aligned}
\mathcal{C}_{\text {spin }}^{c} \stackrel{\cong}{\longrightarrow} \coprod_{X_{0}}\left[T\left(X_{0}\right) / G_{C}\left(X_{0}\right)\right], \\
\mathcal{C}_{\text {spin }}^{c, 0} \stackrel{\cong}{\longrightarrow} \coprod_{X_{0}}\left[* / G_{C}\left(X_{0}\right)\right],
\end{aligned}
$$

where the disjoint union is over a complete set of representatives of $C$-diffeomorphism classes of Spin-surfaces. For a given $X_{0}$, we have a short exact sequence of mapping class groups

$$
1 \rightarrow G^{\text {spin }} \rightarrow G_{C} \rightarrow \prod_{C \in \partial X} \mathbb{Z} / 2 \rightarrow 1
$$

Note that this short exact sequence does not always split. A group $S_{C}$ is defined by the short exact sequence

$$
1 \rightarrow G_{0}^{\text {spin }} \rightarrow G_{C} \rightarrow S_{C} \rightarrow 1
$$


Then we also have a short exact sequence

$$
1 \rightarrow S^{\text {spin }} \rightarrow S_{C} \rightarrow \prod_{C \in \partial X} \mathbb{Z} / 2 \rightarrow 1
$$

and an equivalence of stacks

$$
\mathcal{C}_{\text {spin }}^{c, t o p} \stackrel{\cong}{\longrightarrow} \coprod_{X_{0}}\left[* / S_{C}\left(X_{0}\right)\right]
$$

Similar discussions apply as for $\mathcal{C}, \mathcal{C}_{\text {spin }}$. The treatment of the stack with $2_{C}$-sewing $\mathcal{C}_{\mathbb{Z} / 2}^{c}$ is analogous. One can then investigate $\mathbb{C}^{\times}$-central extensions of $\mathcal{C}_{\text {spin }}^{c}, \mathcal{C}_{\mathbb{Z} / 2}^{c}, \mathcal{C}_{\text {spin }}^{c, 0}, \mathcal{C}_{\mathbb{Z} / 2}^{c, 0}, \mathcal{C}_{\text {spin }}^{c, \text { top }}$, $\mathcal{C}_{\mathbb{Z} / 2}^{c, t o p}$

\section{Conformal field theories}

The purpose of this section is to review the definition of conformal field theory and its variants, and related concepts needed to understand the present paper. Our reference is Segal [21]. Unfortunately, the full version of [21] is not yet published, and this forces us to review some aspects of that theory.

Let $D$ be $\mathbb{C}^{\times}$-central extension of the moduli space $\mathcal{C}$. We shall define conformal field theory (abbr. CFT) with conformal anomaly $D$.

Remark. - It should be remarked that there is a more general notion of conformal anomalies which are allowed to be vector spaces of higher dimension. This is encoded in the notion of modular functor described in [21] (although to get complete gluing data, the set of labels described as a 'set' in [21] should be a groupoid). This general notion of CFT whose conformal anomaly is a modular functor should be equivalent to the notion of vertex operator algebra [10], although infinite-dimensional modular functors (in a suitable sense) would have to be allowed if the vertex operator algebra is irrational. (A vertex operator algebra is called rational essentially if it has only finitely many non-isomorphic modules. For a precise definition, see [8,9].)

In this paper, we will restrict attention to CFT's whose conformal anomaly is 1-dimensional. We will give some examples following Segal [21].

To define a conformal field theory, Segal [21] starts with a Hilbert space $H$. To every conformal surface $X$, and element $\zeta \in D_{X}$, one assigns an element

$$
U(X, \zeta) \in \bigotimes_{\text {out }} H \otimes \bigotimes_{\text {in }} \bar{H}
$$

where the tensor products (= Hilbert tensor products) are taken over the outgoing and incoming components of $\partial X$, and $\bar{H}$ is the dual of $H$. (Since not all of our examples preserve the Hilbert structure, it may be more appropriate to refer to Hilbertizable vector spaces and replace $\bar{H}$ by $H^{*}$ in (3.1).) Note that an element of the form (3.1) can be interpreted as a Hilbert-Schmidt map $[16,22]$

$$
\bigotimes_{\text {in }} H \rightarrow \bigotimes_{\text {out }} H
$$

We require however more strongly that this map be trace class. Then we require that

$$
U\left(X \amalg Y, \zeta_{1} \otimes \zeta_{2}\right)=U\left(X, \zeta_{1}\right) \otimes U\left(Y, \zeta_{2}\right),
$$


and

$$
U(\check{X}, \zeta)=\operatorname{trace}(U(X, \zeta))
$$

One usually puts in more requirements, such as unitarity (reflection positivity); they will, however, not affect the formulas presented here. We will, on the other hand, restrict attention to holomorphic conformal field theories, which means that the operator $U(X, \zeta)$ depends holomorphically on $X, \zeta$ : throughout the rest of this paper, a conformal field theory will automatically mean a holomorphic CFT.

We will be interested in the partition function of a conformal field theory. For $q \in \mathbb{C},\|q\|<1$, we consider the annulus $A_{q}$ which is the region in $\mathbb{C}$ bounded by the circles $\|z\|=1$ and $\|z\|=\|q\|$. The circle $\|z\|=1$ is parametrized by the identity (incoming). The circle $\|z\|=\|q\|$ is parametrized by the map $z \mapsto q z$ (outgoing). Note that there is a canonical element $\iota_{q} \in D_{A_{q}}$ : considering the unit disk $D$ with boundaty parametrized by the identity (incoming), then sewing $A_{q}$ with $D$ gives $D . \iota_{q}$ is the element of $D_{A_{q}}$ which induces the identity on $D_{D}$. Now the partition function is defined by

$$
Z(\tau)=\operatorname{trace}\left(U\left(A_{q}, \iota_{q}\right)\right)
$$

where $q=\mathrm{e}^{2 \pi i \tau}$. Under our assumptions, this is a holomorphic function of $\|q\|<1$.

Let $E_{q}$ be the elliptic curve defined by gluing the two boundary components of $A_{q}$. Segal [21], Proposition 6.9, gives a formula for the modularity of partition functions of holomorphic conformal field theories. Our main goal will be to study variations of this formula. To fix ideas, we give the statement and proof of the formula.

Assume that our holomorphic conformal field theory has central charge $c$, which means that

$$
D \cong D e t^{-c / 2} .
$$

By Theorem 2.21, we may assume $c \in 2 \mathbb{Z}$ in the case of holomorphic CFT's with 1-dimensional anomaly, which we are presently considering. Without referring to Mumford's theorem, the holomorphic $\mathbb{C}^{\times}$-central extension $D$ of $\mathcal{C}$ restricts to a holomorphic central extension of the semigroup of annuli $\mathcal{A}$. Segal [21], Chapter 5, remarks that such holomorphic central extensions are in bijective correspondence with $\mathbb{C}^{\times}$-central extensions of $D$ iff $+\left(S^{1}\right)$, which are classified by central charge $c$ and rotation $h$. The central charge in this sense of Det is -2 , and hence if we define central charge of a CFT as the central charge of $D$, this gives (3.2). More explanation will be given in Section 4 below. Let $q=\mathrm{e}^{2 \pi i \tau}, \operatorname{Im}(\tau)>0$. Then $E_{q} \cong \mathbb{C} /[\tau, 1]$. If

$$
\tau^{\prime}=(a \tau+b) /(c \tau+d), \quad g=\left(\begin{array}{ll}
a & b \\
c & d
\end{array}\right) \in S L_{2}(\mathbb{Z}), \quad q^{\prime}=\mathrm{e}^{2 \pi i \tau^{\prime}}
$$

then $A_{q}$ changes to $A_{q^{\prime}}$ but $E_{q}$ does not change, so

$$
Z\left(\tau^{\prime}\right)=\rho(\tau, g)^{-c / 2} Z(\tau)
$$

where $\rho(\tau, g)$ is the ratio of the image $\varepsilon_{q^{\prime}}$ of $\iota_{q^{\prime}}$ to the image $\varepsilon_{q}$ of $\iota_{q}$ in $\operatorname{Det}\left(E_{q}\right)=\operatorname{Det}\left(E_{q^{\prime}}\right)$, where here $\iota_{q}$ is the canonical element of $\operatorname{Det}\left(A_{q}\right)$.

THEOREM 3.3 (Segal [21], 6.9). - We have

$$
\rho(\tau, g)=u^{2}(g) \mathrm{e}^{2 \pi i\left(\tau-\tau^{\prime}\right) / 12}
$$

where $u^{2}$ is as described in the beginning of Section 7. 
Remark. - In Section 7, we will describe $u$ as a homomorphism on a central extension of $S L_{2}(\mathbb{Z})$ by $\mathbb{Z} / 2$, but the square of the map vanishes on the center (as is true for any homomorphism).

COROLLARY 3.4. - For a conformal field theory with central charge $c$,

$$
\frac{Z\left(\tau^{\prime}\right)}{Z(\tau)}=\frac{\left(q^{\prime}\right)^{c / 24}}{q^{c / 24}} u^{-c}(g)
$$

Proof of Theorem 3.3. - To prove the theorem, we first need a description of $\operatorname{Det}\left(E_{q}\right)$ which would be invariant under modular transformations. To this effect, we consider diagram (2.18) with $X=A_{q}, \check{X}=E_{q}$. The top line is

$$
\operatorname{Hol}\left(E_{q}\right) \rightarrow \operatorname{Hol}\left(A_{q}\right) \rightarrow \Omega^{0}\left(S^{1}\right)
$$

We see that, since $\operatorname{Hol}\left(E_{q}\right)$ is canonically isomorphic to $\mathbb{C}$, the determinant line is dual to the determinant line of the cokernel of the right hand map (3.5).

At this point, it is time to establish notation for sheaves of forms. We denote by $\Omega^{n}\left(S^{1}\right)$ ordinary de Rham forms on $S^{1}$. For a conformal surface $X$, we shall denote by $\Omega^{p, q}(X)$ the $\mathbb{C}$-vector space of de $\operatorname{Rham}(p, q)$-forms on $X$. (Obviously, only very small values of $p, q, n$ are relevant.) By underlining any of these notations, we shall mean the corresponding sheaf of forms (in the analytic topology). Conversely, for a sheaf, sections will be denoted by $\Gamma$. A subscript $H o l$ will indicate holomorphic sections. The embedding $S^{1} \rightarrow E_{q}$ will be denoted by $i$.

Then we have an exact sequence of sheaves on $E_{q}$

$$
0 \rightarrow \underline{\Omega_{H o l}^{0,0}\left(E_{q}\right)} \rightarrow \underline{\Omega_{H o l}^{0,0}\left(A_{q}\right)} \rightarrow i_{*} \underline{\Omega^{0}\left(S^{1}\right)} .
$$

Now (3.5) is a part of the long exact sequence in cohomology corresponding to (3.6), and hence the cokernel of the right hand map (3.5) is

$$
H^{1}\left(E_{q}, \underline{H o l}\right)=H^{0,1}\left(E_{q}\right) .
$$

This is 1-dimensional, so we have shown that

$$
\operatorname{Det}\left(E_{q}\right) \cong\left(H^{0,1}\left(E_{q}\right)\right)^{-1}
$$

Now let $\delta$ be the connecting map of the long exact sequence in cohomology induced by (3.6). Define

$$
\xi_{q}=\delta(1)^{-1}
$$

(A non-zero element of a complex line has a well defined reciprocal in the dual line.) To determine the behaviour of $\xi_{q}$ under modular transformations, we must determine $\delta(1)$ explicitly. 
To this end, consider the diagram

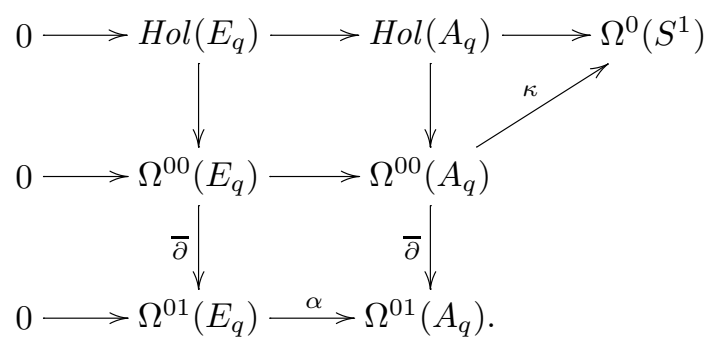

The map $\kappa$ is onto, and a $(0,1)$-form on $E_{q}$ representing $\delta(1)$ can be obtained as

$$
\alpha^{-1} \bar{\partial} \kappa^{-1}(1)
$$

By picking a representative of $\kappa^{-1}(1)$ which is $\mathbb{R}$-linear and constant in the real direction, it is easily seen that such a form is a constant times

$$
(\operatorname{Im}(\tau))^{-1} d \bar{u}
$$

where $u$ is the standard coordinate in $\mathbb{C}$, where we consider $E_{q}=\mathbb{C} /[\tau, 1]$. Now for

$$
g=\left(\begin{array}{ll}
a & b \\
c & d
\end{array}\right) \in S L_{2}(\mathbb{Z}), \quad g(\tau)=\tau^{\prime},
$$

it is well known that, since $\operatorname{Im}(\tau)$ is proportional to volume,

$$
\operatorname{Im}\left(\tau^{\prime}\right)=\operatorname{Im}(\tau) /\|c \tau+d\|^{2} .
$$

On the other hand, obviously,

$$
d \overline{u^{\prime}}=\frac{d \bar{u}}{\overline{c \tau+d}}
$$

We conclude that, if $q^{\prime}=\mathrm{e}^{2 \pi i \tau^{\prime}}$,

$$
\xi_{q^{\prime}}=\xi_{q} /(c \tau+d) .
$$

To finish the proof of the theorem, we must relate the element $\varepsilon_{q}$ to $\xi_{q}$. But this is now easy: By the definition of $\xi_{q}$, We have

$$
\varepsilon_{q}=\xi_{q} \cdot \operatorname{det}(F)
$$

where

$$
F: \operatorname{Ker}(\delta) \rightarrow \operatorname{Ker}(\delta)
$$

is the operator which sends $\pi_{A_{q}}\left(z^{n}\right)$ to $\tilde{\pi}\left(z^{n}\right)$. We see that

$$
F\left(z^{n}\right)=\left(1-q^{n}\right) z^{n} \quad \text { for } n>0
$$

and

$$
F\left(z^{-n}\right)=\left(1-q^{n}\right) z^{-n} \text { for } n>0
$$


Thus,

$$
\varepsilon_{q}=\xi_{q} \prod_{n>0}\left(1-q^{n}\right)^{2}
$$

and the statement of the theorem follows from the modularity properties of the $\eta$-function (7.2):

$$
\frac{\varepsilon_{q^{\prime}}}{\varepsilon_{q}}=\frac{\xi_{q^{\prime}}}{\xi_{q}} \cdot \frac{\eta\left(\tau^{\prime}\right) \cdot\left(q^{\prime}\right)^{-1 / 12}}{\eta(\tau) \cdot q^{-1 / 12}}=\frac{1}{c \tau+d} \cdot(c \tau+d) \cdot u^{2}(g) \frac{\left(q^{\prime}\right)^{-1 / 12}}{q^{-1 / 12}} .
$$

Note that the conclusion of the theorem implies that $Z(\tau)=0$ if $\tau=i$ or $\tau=\sqrt[3]{1}$ (because then there exists a level 1 modular transformation $g$ such that $g(\tau)=\tau$, while $\left.u^{2}(g) \neq 1\right)$. Thus, we have the following somewhat peculiar

COROLLARY 3.11. - For a holomorphic conformal field theory of central charge not divisible by 2 and 3 , we have $Z\left(\mathrm{e}^{-2 \pi}\right)=Z\left(-\mathrm{e}^{-\sqrt{3} \pi / 2}\right)=0$.

Remark. - There is a vertex operator algebra [10] with central charge 24 , and partition function

$$
Z(\tau)=q \cdot(j(\tau)-744)
$$

and an action of the Monster. This formula suggests that this vertex operator algebra should give rise to a CFT with 1-dimensional conformal anomaly. This would be a most interesting example from the point of view of elliptic cohomology. It is not known to the author if this is true, although some progress in this direction follows from the ideas of [3].

One of the purposes of this paper is to generalize the modularity formula (3.4). To this end, we shall define a conformal field theory with Spin-structure (Spin-CFT), as a pair of Hilbert spaces $H_{A}$ and $H_{P}$ (spaces of states corresponding to $S_{A}^{1}$ and $S_{P}^{1}$ ), a $\mathbb{C}^{\times}$-central extension $D$ of $\mathcal{C}_{\text {spin }}$, and a map assigning to every conformal surface with Spin-structure $X$ and element $\alpha \in D_{X}$ an element

$$
U(X, x) \in \bigotimes_{\text {out }, A} H_{A} \otimes \bigotimes_{\text {out }, P} H_{P} \otimes \bigotimes_{\text {in }, A} \overline{H_{A}} \otimes \bigotimes_{\text {in }, P} \overline{H_{P}}
$$

(the product is over incoming and outgoing, antiperiodic and periodic boundary components).

To define partition function for Spin-CFT, note that there are two semigroups of annuli (with one inbound and one outbound boundary component) contained in $\mathcal{C}_{\text {spin }}$, depending on whether the Spin-structure is antiperiodic or periodic. The semigroup $\mathcal{A}_{A}$ of antiperiodic annuli is a 2 -sheeted cover of $\mathcal{A}$, while the semigroup $\mathcal{A}_{P}$ of periodic annuli is $\mathcal{A} \times \mathbb{Z} / 2$.

We will prove in the next section that a $C^{\times}$-central extension of $\mathcal{C}_{\text {spin }}$ splits canonically over the sub-semigroup of $\mathcal{A}_{A}$ consisting of the standard annuli $A_{q}$. Thus, if $\iota$ is the canonical element of $D_{A_{q}}$, we may define

$$
Z(\tau)=\operatorname{tr}\left(U\left(A_{q}, \iota\right)\right)
$$

$q=\mathrm{e}^{2 \pi i \tau}$. Note, however, that unlike the case of a CFT, $Z(\tau)$ will depend on a choice of $q^{1 / 2}$, which corresponds to the choice of spin of the boundary parametrization of $A_{q}$.

Finally, define conformal field theory with square root structure (Sqrt-CFT) analogously as a Spin-CFT, with $\mathcal{C}_{\text {spin }}$ replaced by $\mathcal{C}_{\mathbb{Z} / 2}$. To define partition function for $S q r t$-CFT's, we will show in the next section that a $\mathbb{C}^{\times}$-central extension of $\mathcal{C}_{\mathbb{Z} / 2}$ splits canonically when restricted to the sub-semigroup of the standard annuli in $\mathcal{A}_{P} \cong \mathcal{A} \times \mathbb{Z} / 2$. If we denote these standard annuli by $\left(A_{q}, \varepsilon\right), \varepsilon \in \mathbb{Z} / 2$, then we have a canonical element $\iota_{\varepsilon} \in D_{\left(A_{q}, \varepsilon\right)}$. We define

$$
Z_{\varepsilon}(\tau)=\operatorname{tr}\left(U\left(\left(A_{q}, \varepsilon\right), \iota_{\varepsilon}\right)\right)
$$


We will now discuss the fundamental example of [21], i.e. fermionic Fock space conformal field theories. For a polarized Hilbert space $H$, one can consider the fermionic Fock space $\mathcal{F}(H)$, which is the Hilbert completion of the exterior algebra

$$
\Lambda\left(H_{+} \oplus \overline{H_{-}}\right) .
$$

(The Fock space should be thought of as a Floer-like analogue of the exterior algebra of a finitedimensional vector space. Along these lines, other, more invariant definitions of the Fock space exist: for example, it can be defined as a module of spinors of the Clifford algebra of the space $H \oplus \bar{H}$ with respect to a choice of vacuum vector - see e.g. [16], p. 257.)

Now Segal [21] constructs, for each $k \in \mathbb{Z}$, a CFT $\mathcal{F}\left(\Omega^{k}\right)$ with $H=\mathcal{F}\left(\Omega^{k}\left(S^{1}\right)\right)$, and a Spin-CFT $\mathcal{F}\left(\Omega^{k+\frac{1}{2}}\right)$ with $H=\mathcal{F}\left(\Omega^{k+\frac{1}{2}}\left(S^{1}\right)\right)$. Roughly, the space of holomorphic $\alpha$-forms on $X\left(\alpha=k\right.$ or $\left.k+\frac{1}{2}\right)$ is interpreted as a graph of a partial function from the $\alpha$-forms on the incoming boundary components to the $\alpha$-forms on the outgoing boundary components. These graphs are composed as partial functions, and define rays in the Fock space of $\alpha$-forms on $\partial X$.

To understand the situation in more detail, we must first consider the Grassmanian composition in the finite case (see [21]). Consider the following situation. Let $H_{1}, H_{2}$ be finite-dimensional $\mathbb{Z}$-vector spaces. Let $V \subset H_{1} \oplus H_{2} \oplus H_{2}$. Define $\sigma(V) \subset H_{1}$ by

$$
\sigma(V)=\left\{v_{1} \mid \text { there exists } v_{2} \in H_{2} \text { such that }\left(v_{1}, v_{2},-v_{2}\right) \in V\right\},
$$

provided that the following conditions are satisfied:

$$
\text { The map } V \rightarrow H_{1} \oplus H_{2} \text { defined by }\left(v_{1}, v_{2}^{\prime}, v_{2}^{\prime \prime}\right) \mapsto\left(v_{1}, v_{2}^{\prime}+v_{2}^{\prime \prime}\right) \text { is injective, }
$$

The map $V \rightarrow H_{2}$ defined by $\left(v_{1}, v_{2}^{\prime}, v_{2}^{\prime \prime}\right) \mapsto v_{2}^{\prime}+v_{2}^{\prime \prime}$ is surjective.

We consider $\sigma(V)$ undefined if either one of the conditions (3.13) or (3.14) fails.

Proposition 3.15. - Let $\operatorname{dim}\left(H_{2}\right)=n$. Consider the composition

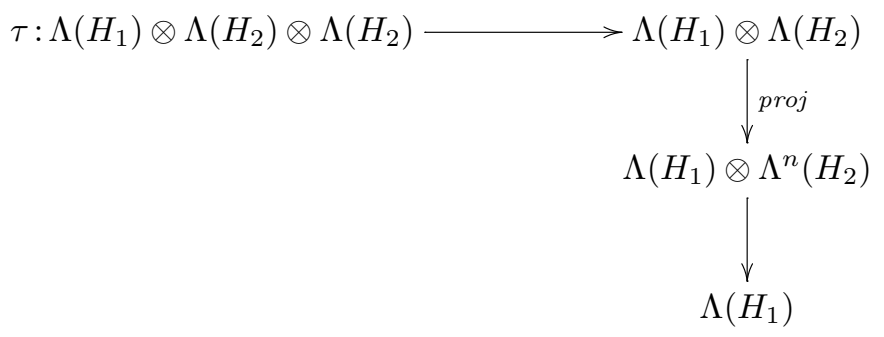

where the first arrow is induced by addition $\mathrm{H}_{2} \oplus \mathrm{H}_{2} \rightarrow \mathrm{H}_{2}$ and the last arrow is given by choosing a non-zero element in the dual of the 1-dimensional space $\Lambda^{n}\left(H_{2}\right)$. Denote, also, for $V \subset H, \lambda V \in \Lambda(H)$ the determinant defined up to scalar multiple (i.e. a non-zero element of the determinant line of $V$ ). Then

$$
\tau(\lambda(V))= \begin{cases}c . \lambda(\sigma(V)) & \text { if } \sigma(V) \text { is defined } \\ 0 & \text { else. }\end{cases}
$$

Proof. - We let $\nabla \subset H_{2} \oplus H_{2}$ be the antidiagonal consisting of elements of the form $(v,-v)$. Let $H_{20} \subset H_{1} \oplus H_{2} \oplus H_{2}=H$ be the first copy of $H_{2}$. Write $H=H_{1} \oplus \nabla \oplus H_{20}$. Reduced 
row echelon form then implies that there exists a basis of $V$ of the following form:

$$
\begin{array}{ll}
\left(r_{i}, s_{i}, t_{i}\right) \in H_{20} \oplus H_{1} \oplus \nabla, & i=1, \ldots, m, \\
\left(0, s_{i}, t_{i}\right) \in H_{1} \oplus \nabla, & i=m+1, \ldots, n, \\
\left(0,0, t_{i}\right) \in \nabla, & i=n+1, \ldots, p,
\end{array}
$$

where the sets

$$
\left\{r_{i} \in H_{20} \mid i=1, \ldots, m\right\}, \quad\left\{s_{i} \in H_{1} \mid i=m+1, \ldots, n\right\}, \quad\left\{t_{i} \in \nabla \mid i=n+1, \ldots, p\right\}
$$

are linearly independent. However, condition (3.14) says that $\left\{r_{i}\right\}$ is a basis of $H_{20}$, and condition (3.13) says that $p=n$. Obviously, if either of these assumptions fails, then $\tau(\lambda(V))=0$. If they are satisfied, then the surviving terms of the exterior product $\lambda(V)$ of (3.16) after applying the product $\Lambda\left(H_{2} \oplus H_{2}\right) \rightarrow \Lambda\left(H_{2}\right)$ are

$$
\bigwedge_{i=1}^{m} r_{i} \wedge \bigwedge_{i=m+1}^{n} s_{i} .
$$

After applying the next two maps in the definition of $\tau$, we obtain

$$
\bigwedge_{i=m+1}^{n} s_{i}
$$

which is $\lambda(\sigma(V))$.

We now shift attention to the infinite-dimensional case. Suppose $H_{1}$ and $H_{2}$ are polarized Hilbert spaces. For a polarized Hilbert space $H$, let $\widetilde{H}$ denote the same Hilbert space with reversed polarizaton. Now suppose $V \in G r\left(H_{1} \oplus H_{2} \oplus \widetilde{H_{2}}\right)$ where $G r(H)$ denotes the restricted Grassmanian of $H$, consisting of all closed subspaces $V$ such that the projection $V \rightarrow H_{+}$ is Fredholm, and the projection $V \rightarrow H_{-}$is Hilbert-Schmidt (see [16]). Define $\sigma(V)$ by the formula (3.12) if the conditions (3.13) and (3.14) are satisfied, and consider it undefined otherwise.

Next, we must define the infinite analogue of the map $\tau$. The Fock space $\mathcal{F}(H)$ of a polarized Hilbert space $H$ is the Hilbert completion of $\Lambda\left(H_{+} \oplus \overline{H_{-}}\right)$. Note that the Fock space $\mathcal{F}\left(\widetilde{H_{2}}\right)$ is Hilbert-dual to $\mathcal{F}\left(H_{2}\right)$. Thus, we have a "supertrace map"

$$
\mathcal{F}\left(\widetilde{H_{2}}\right) \otimes \mathcal{F}\left(H_{2}\right) \rightarrow \mathbb{C}
$$

or

$$
\text { str }: \mathcal{F}\left(\widetilde{H_{1}}\right) \otimes \mathcal{F}\left(\widetilde{H_{2}}\right) \otimes \mathcal{F}\left(H_{2}\right) \rightarrow \mathcal{F}\left(\widetilde{H_{1}}\right) .
$$

The supertrace is defined in the same way as the trace, but with reversed signs in odd degrees. In this formula, the supertrace arises because of signs which occur when we permute elements in exterior products. This map cannot be extended to the Hilbert tensor product, but does extend to its subspace of trace class elements [22]. Now for $V \in G r(H)$, we have the Plücker coordinate $\lambda V \in \mathcal{F}(\widetilde{H})$ (see [16]), defined up to scalar multiple. The appropriate infinite-dimensional analogue of Proposition 3.15 is then

$4^{\mathrm{e}}$ SÉRIE - TOME $36-2003-\mathrm{N}^{\circ} 1$ 
Proposition 3.17. - Let $V \in G r\left(H_{1} \oplus H_{2} \oplus \widetilde{H_{2}}\right)$. Then, up to non-zero scalar multiple,

$$
\operatorname{str}(\lambda(V))= \begin{cases}0 & \text { if } V \text { does not satisfy (3.13) or (3.14), } \\ \lambda(\sigma(V)) & \text { else. }\end{cases}
$$

Proof. - Analogous to the finite-dimensional case, using the methods of [16], Section 10.6.

Finally, consider the case of $\alpha$-forms. If $X$ is a connected conformal surface with nonempty boundary (and Spin-structure if $\alpha \in \frac{1}{2}+\mathbb{Z}$ ), then we can consider the space $\Omega^{\alpha}(X)$ of holomorphic $\alpha$-forms on the interior of $X$ which extend to $L^{2}$-forms on the boundary of $X$. For $\alpha \in \mathbb{Z}$, putting $H=\Omega^{\alpha}\left(S^{1}, \mathbb{C}\right)$, the space of $\mathbb{C}$-valued $L^{2}$ - $\alpha$-forms on $S^{1}$, this identifies

$$
\Omega^{\alpha} X \in G r\left(\bigoplus_{\text {in }} \tilde{H} \oplus \bigoplus_{\text {out }} H\right) .
$$

In the case $\alpha \in \frac{1}{2}+\mathbb{Z}$, this is altered in the obvious way to account for Spin-structure on the boundary components of $X$. The inclusion

$$
\Omega^{\alpha} X \subset \bigoplus_{\text {in }} \tilde{H} \oplus \bigoplus_{\text {out }} H
$$

is the restriction to the boundary, composed with a minus sign on incoming boundary components. If $X$ is connected and $\partial X \neq \emptyset$, we let

$$
U(X)=\lambda\left(\Omega^{\alpha} X\right) \in \widehat{\bigotimes}_{\text {in }} \mathcal{F}(\widetilde{H}) \hat{\otimes} \widehat{\bigotimes}_{\text {out }} \mathcal{F}(H)
$$

Again, the obvious change is made when $\alpha \in \frac{1}{2}+\mathbb{Z}$. Note that $U(X)$ is defined only up to scalar multiple, so we have defined a CFT with conformal anomaly. The fact that (3.19) satisfies the axioms of CFT at least when $X$ has no closed components follows directly from Proposition 3.17 and from the obvious fact that

$$
\Omega^{\alpha} \check{X}=\sigma\left(\Omega^{\alpha} X\right)
$$

when $\check{X}$ is obtained from $X$ by sewing. If $X$ is closed, then we let $D_{X}$ be $\mathbb{C}$ if $H^{*}\left(X, \Omega^{\alpha}\right)=0$. Otherwise, we put $D_{X}=\operatorname{Det}\left(H^{*}\left(X, \Omega^{\alpha}\right)\right)$, and $U(X)=0$.

One caveat emptor is that, in $H=\mathcal{F}\left(\Omega^{\alpha}\left(S^{1}\right)\right)$, we have grading by exterior degree, and we have, actually, constructed graded conformal field theories, which means that each time we permute factors of degree $p$ and $q$, we multiply by $(-1)^{p q}$. We also must replace trace by supertrace in the definition of partition function.

Comment. - One can also consider a different concept of super-conformal field theory, which have even more structure. The Lie algebra $\operatorname{Vect}\left(S^{1}\right)$ of vector fields on $S^{1}$ can be thought of as the Lie algebra of Diff $\left(S^{1}\right)$. The Lie-theoretic version of universal central extension of $\operatorname{Diff}\left(S^{1}\right)$ is the Virasoro algebra. Conformal field theory can, in some sense, be thought of as additional structure on representations of the Virasoro algebra.

But $\operatorname{Vect}\left(S^{1}\right)$ fits together with the space of $-1 / 2$-forms $\Omega^{-1 / 2}\left(S^{1}\right)$ into a super-Lie algebra (graded Lie algebra) structure on $\operatorname{Vect}\left(S^{1}\right) \oplus \Omega^{-1 / 2}\left(S^{1}\right)$. The universal central extension of this super-Lie algebra is the super-Virasoro algebra. Now a super-conformal field theory is a concept relating to representations of super-Virasoro algebra in the same way as conformal field theories relate to representations of Virasoro algebra. 
A mathematical treatment of super-conformal field theories is not included in [21], but can be worked out using super-Riemann surfaces $[20,1,11,19]$. The super-Riemann surfaces form a super-moduli space. When considering only the space directions (and not "super-directions") in the super-moduli space, super-Riemann surfaces essentially reduce just to Riemann surfaces with Spin-structure (which are treated in [21]).

However, we need to return to the Fock space, and calculate the partition function of $\mathcal{F}\left(\Omega^{\alpha}\left(S^{1}\right)\right)$. We consider the variable $z$ on the incoming boundary component of $A_{q}(\|z\|=1)$, and the variable $t=z / q$ on the outgoing boundary component $(\|z\|=\|q\|)$. The space of holomorphic $\alpha$-forms has admissible basis

$$
\begin{array}{ll}
\left(z^{n}(d z)^{\alpha},-q^{n+\alpha} t^{n}(d t)^{\alpha}\right) & \text { for } n \geqslant 0, \\
\left(-q^{n-\alpha} z^{-n}(d z)^{\alpha}, t^{-n}(d t)^{\alpha}\right) & \text { for } n>0 .
\end{array}
$$

The corresponding element of the determinant line of $\Omega^{\alpha}\left(A_{q}\right)$ is

$$
\bigwedge_{n \geqslant 0}\left(z^{n}(d z)^{\alpha}-q^{n+\alpha} t^{n}(d t)^{\alpha}\right) \wedge \bigwedge_{n>0}\left(-q^{n-\alpha} z^{-n}(d z)^{\alpha}-t^{-n}(d t)^{\alpha}\right) .
$$

The above discussion says that to calculate the supertrace, we don't need to convert to Fock space notation, but can simply set $z=t$ in (3.21) and read off the coefficient in the sum (3.21) at

$$
\bigwedge_{n \geqslant 0} z^{n}(d z)^{\alpha} \wedge \bigwedge_{n>0} z^{-n}(d z)^{\alpha}
$$

The answer is

$$
Z(\tau)=\prod_{n \geqslant 0}\left(1-q^{n+\alpha}\right) \prod_{n>0}\left(1-q^{n-\alpha}\right)
$$

and, for $\alpha \in \mathbb{Z}$,

$$
Z_{-}(\tau)=\prod_{n \geqslant 0}\left(1+q^{n+\alpha}\right) \prod_{n>0}\left(1+q^{n-\alpha}\right)
$$

In particular, for $\alpha \in \mathbb{Z}$,

$$
Z(\tau)=0 .
$$

Thus, in this case, the statement of Corollary 3.4 holds for trivial reasons.

Processing (3.22) and (3.23) further, we get, for $\alpha \in \mathbb{Z}$,

$$
Z_{-}(\tau)=q^{\frac{1}{2} \alpha(1-\alpha)} \prod_{n \geqslant 0}\left(1+q^{n}\right)^{2}=q^{\frac{1}{24}(12 \alpha(1-\alpha)-2)} \cdot\left(2 q^{1 / 12} \prod_{n>0}\left(1+q^{n}\right)^{2}\right),
$$

and for $\alpha \in \frac{1}{2}+\mathbb{Z}$,

$$
\begin{aligned}
Z(\tau) & = \pm q^{-\frac{1}{2}\left(\alpha+\frac{1}{2}\right)^{2}} \prod_{n \geqslant 0}\left(1-q^{n+\frac{1}{2}}\right)^{2} \\
& =q^{\frac{1}{24}(12 \alpha(1-\alpha)-2)} \cdot\left(q^{-1 / 24} \prod_{n \geqslant 0}\left(1-q^{n+\frac{1}{2}}\right)^{2}\right) .
\end{aligned}
$$


The significance of the first factor on the right hand sides of (3.24), (3.25) is the central charge. A conformal field theory specifies, in particular, a central extension of $\operatorname{Diff}\left(S^{1}\right)$ by $\mathbb{C}^{\times}$([21], Chapter 5). These extensions have an invariant called central charge. Segal [21], Chapter 8, calculates the central charge of the conformal field theory $\mathcal{F}\left(\Omega^{\alpha}\right)$ to be

$$
c=12 \alpha(1-\alpha)-2
$$

This explains the factor of -2 in the exponent of (3.2), since for $\alpha=0$, the holomorphic ray in $\mathcal{F}\left(\Omega^{\alpha}(\partial X)\right)$ is $\operatorname{Det}(X)$ (see also next section).

\section{4. $\mathbb{C}^{\times}$-central extensions up to equivalence}

The purpose of this section is to calculate the $\mathbb{C}^{\times}$-central extensions of $\mathcal{C}, \mathcal{C}_{\text {spin }}, \mathcal{C}_{\mathbb{Z} / 2}$. The general plan is as follows. Following [21], we introduce a certain invariant of central extensions called central charge $c$. Central extensions with $c=0$ will define flat bundles on the moduli spaces $\mathcal{C}, \mathcal{C}_{\text {spin }}, \mathcal{C}_{\mathbb{Z} / 2}$, and hence $\mathbb{C}^{\times}$-central extensions of $\mathcal{C}^{0}, \mathcal{C}_{\text {spin }}^{0}, \mathcal{C}_{\mathbb{Z} / 2}^{0}$. We then introduce additional invariants called rotation numbers. If the rotation numbers also vanish, it implies trivial actions of the pure modular groups on the line associated to each type of surfaces, and we shall obtain trivial central extensions in the sense introduced above, or $\mathbb{C}^{\times}$-central extensions of $\mathcal{C}^{\text {top }}$, $\mathcal{C}_{\text {spin }}^{\text {top }}, \mathcal{C}_{\mathbb{Z} / 2}^{\text {top }}$. In this section, we shall classify $\mathbb{C}^{\times}$-central extensions up to equivalence, by which we mean modulo trivial $\mathbb{C}^{\times}$-central extensions.

We shall make use of the following results.

LEMMA 4.1. - If $D$ is a holomorphic $\mathbb{C}^{\times}$-central extension of $\mathcal{C}$ which enjoys a functorial holomorphic flat connection compatible with sewing, then $D$ is a pullback of a canonical holomorphic $\mathbb{C}^{\times}$-central extension of $\mathcal{C}^{0}$. If, further, the pure modular groups act trivially on $D$, then $D$ is a pullback of a canonical $\mathbb{C}^{\times}$-central extension of $\mathcal{C}^{\text {top }}$. Similarly for $\mathcal{C}_{\text {spin }}, \mathcal{C}_{\mathbb{Z} / 2}$, $\mathcal{C}_{\text {spin }}^{c}, \mathcal{C}_{\mathbb{Z} / 2}^{c}$.

Proof. - All the cases are analogous, so we only discuss $\mathcal{C}$. The second assertion is obvious from the short exact sequence (2.11). To prove the first assertion, we must show how a diffeomorphism $\phi: X \rightarrow Y$ of conformal surfaces which preserves boundary parametrization defines a canonical map on $D$. To this end, note that our assumption gives a canonical $G(X)$-equivariant flat connection on the Teichmüller space $T(X)$, where $G(X)$ is the mapping class group. The diffeomorphism specifies $Y$ as a point of $Y \in T(X)$. Since $T(X)$ is contractible, the flat connection defines a canonical isomorphism $D_{Y} \cong D_{X}$. The required properties are easily verified.

The following is a well known fact:

LEMMA 4.2. - All elements of the pure mapping class groups $G_{0}(X), G_{0}^{\text {spin }}(X), G_{0}^{\mathbb{Z} / 2}$ can be obtained from identities and elements of the mapping class groups of annuli with one inbound and one outbound boundary component by using the operations of sewing and disjoint union in the respective moduli stack with $K$-sewing $\mathcal{C}, \mathcal{C}_{\text {spin }}, \mathcal{C}_{\text {spin }}^{c}$ or $\mathcal{C}_{\mathbb{Z} / 2}^{c}\left(\right.$ but not $\left.\mathcal{C}_{\mathbb{Z} / 2}\right)$.

The counterexample for $\mathcal{C}_{\mathbb{Z} / 2}$ is the sign reversal on a trivial $S q r t$-structure on a closed conformal surface $X$.

In [21], Segal proves that a functorial flat holomorphic connection compatible with sewing exists on a $\mathbb{C}^{\times}$-central extension $D$ of $\mathcal{C}$, (resp. $\left.\mathcal{C}_{\text {spin }}, \mathcal{C}_{\text {spin }}^{c}, \mathcal{C}_{\mathbb{Z} / 2}^{c}, \mathcal{C}_{\mathbb{Z} / 2}\right)$ if $c=0$ where $c$ is a certain invariant called central charge (which we shall define below). Anyway, the point is that 
the central charge is also determined by the restriction of $D$ to the respective semigroup of annuli. In view of Lemma 4.2, we therefore have

LEMMA 4.3. - Up to equivalence (as defined above), a holomorphic $\mathbb{C}^{\times}$-central extension of $\mathcal{C}, \mathcal{C}_{\text {spin }}, \mathcal{C}_{\text {spin }}^{c}, \mathcal{C}_{\mathbb{Z} / 2}^{c}$ is determined by its restrictions to the semigroups of annuli contained in each corresponding category.

Note that $\mathcal{C}$ contains one semigroup of annuli, while $\mathcal{C}_{\text {spin }}, \mathcal{C}_{\text {spin }}^{c}, \mathcal{C}_{\mathbb{Z} / 2}^{c}, \mathcal{C}_{\mathbb{Z} / 2}$ contain two semigroups of periodic and antiperiodic annuli each.

We will begin by proving Theorem 2.21. Segal [21], Section 5, proves that holomorphic $\mathbb{C}^{\times}$-central extensions of semigroup of annuli $\mathcal{A}$ are in bijective correspondence with $\mathbb{C}^{\times}$-central extensions of Diff $+\left(S^{1}\right)$, which is classified by two numbers $c \in \mathbb{C}$ (the central charge) and $h \in \mathbb{C} / \mathbb{Z}$ (the rotation number). Any pair of these numbers can occur. The rotation number $h \in \mathbb{C} / \mathbb{Z}$ classifies $\mathbb{C}^{\times}$-central extensions of $S^{1}$, and holomorphic $\mathbb{C}^{\times}$-central extension of the semigroup

$$
\mathbb{C}_{<1}^{\times}=\left\{q \in \mathbb{C}^{\times} \mid\|q\|<1\right\} .
$$

Recall from [21] that in the standard basis $L_{n}=\mathrm{e}^{i n \theta} d / d \theta$ of $\operatorname{Vect}\left(S^{1}\right)$, the Lie algebra version of a central extension of $\operatorname{Diff}_{+}\left(S^{1}\right)$ satisfies

$$
\left[L_{-n}, L_{n}\right]=2 i n L_{0}-\frac{1}{12} \operatorname{cn}\left(n^{2}-1\right) .
$$

Furthermore, in a representation of a central extension of $\operatorname{Vect}\left(S^{1}\right), h$ can be characterized as any eigenvalue of $L_{0}$, multiplied by $-i$. (Note: other authors use other conventions; the most common choice is, setting $z=\mathrm{e}^{i \theta}$, to put $L_{n}=-z^{n+1} d / d z$, which is our choice multiplied by $i$.)

Thus, the theorem reduces to finding out which holomorphic $\mathbb{C}^{\times}$-central extensions of $\mathcal{A}$ extend to $\mathbb{C}^{\times}$-central extensions of $\mathcal{C}$. First, we see that for a holomorphic central extension $D$ of $\mathcal{C}$, we have $h=0$, i.e. the central extension is trivial on the semigroup of standard annuli $\mathbb{C}_{<1}^{\times}$. If $D$ is the unit disk with identity (incoming) boundary parametrization, then sewing $D$ with $A_{q}$ gives $D$, which gives an isomorphism

$$
D_{D} \otimes D_{A_{q}} \cong D_{D}
$$

Choose $\iota_{q} \in D_{A_{q}}$ which corresponds to the identity on $D_{D}$. Then, by associativity of sewing, $\iota_{q} \cdot \iota_{q^{\prime}}=\iota_{q q^{\prime}}$, which proves $h=0$.

Now we recall that for $D=\operatorname{Det}$, we have $h=0, c=-2$. Thus, we are done if we can prove

PROPOSITION 4.5. - For a holomorphic central extension $D$ of $\mathcal{C}$, we have $2 \mid c$.

Proof. - We will consider the elliptic curve $E_{\tau}=\mathbb{C} /[\tau, 1]$, and the curve $E_{\tau^{\prime}}$ obtained from $E_{\tau}$ by removing a small circle with center 0 and radius $\varepsilon$, parametrized by $t=z \varepsilon$. More generally, if $L$ is any lattice in $\mathbb{C}$, we may analogously consider $E_{L}, E_{L}^{\prime}$. Let $\alpha=E_{i}^{\prime}$. Then in the moduli space $\mathcal{C}_{\alpha}$, we will consider the following loops at $\alpha$ :

$$
f_{i}(t)=E_{L(t)}^{\prime}, \quad t \in[0,1], i=1,2,3
$$

where

$$
L_{1}(t)=[i+t, 1], \quad L_{2}(t)=[1+i, 1-t(1+i)], \quad L_{3}(t)=[1+t i,-i] .
$$


Then the loops $f_{i}$ are Dehn twists, which means that they are obtained by cutting $\alpha$ along a simple curve, and gluing back with a shift of parametrization on the curve. (The curves $f_{1}, f_{2}, f_{3}$ are the line segments $[i, 1+i],[1,-i],[1+i, 1]$, respectively.) Thus, because the rotation number of $D$ is $0, D$ splits canonically on the curves $f_{i}$. In other words, there is a canonical section of $D$ along the loop $f_{3} f_{2} f_{1}$.

But now $f=\left(f_{3} f_{2} f_{1}\right)^{4}$ is homotopic to the loop at $\alpha$ in $\mathcal{C}_{\alpha}$ which leaves $\alpha$ fixed, except rotating the parametrization of its boundary $360^{\circ}$. Now the tangent space to $\mathcal{C}_{E_{L}^{\prime}}$ at $E_{L}^{\prime}$ is the space of $\mathbb{C}$-vector fields on $\partial E_{L}^{\prime}$ modulo vector fields which extend to $E_{L}^{\prime}$ holomorphically. Thus, $f$ is equal to a path in $\mathcal{C}_{\alpha}$ obtained by leaving the curve $E_{[i, 1]}$ fixed, and varying the disk $D_{t}$ we are cutting out, with its boundary parametrization. It follows that

$$
\frac{f(1)}{f(0)}=\mathrm{e}^{2 \pi i c \varrho}
$$

for some constant $\varrho$, independent of the choice of $D$.

LEMMA 4.7. $-\varrho=-1 / 2$.

Suppose the lemma has been proven. Then note that we must have

$$
1=\frac{f(1)}{f(0)}
$$

since $f$ is a concatenation of Dehn twists, and the rotation number is 0 . Thus, $\mathrm{e}^{2 \pi i c \varrho}=1$, and hence $2 \mid c$, as claimed.

Proof of Lemma 4.7. - Note that, theoretically, the boundary parametrization of the disk $D_{t}$ can be calculated using Weierstrass' $\zeta$-function, which would give an explicit formula for $f(t)$. However, this seems tedious.

Instead, note that the statement can be phrased in terms of $L \mid \mathcal{A}$, which can be logarithmed in the sense that it comes from a $\mathbb{C}$-Lie algebra extension. However, the different $\mathbb{C}$-Lie algebra extensions of $\operatorname{Vect}_{\mathbb{C}}\left(S^{1}\right)$ differ only by a multiplicative constant in their cocycle. Thus, it suffices to prove the statement for $D=$ Det.

Now for $D=D e t$, use the isomorphism obtained by sewing a unit disk to $D_{t}$ :

$$
\operatorname{Det}\left(E_{f(t)}^{\prime}\right) \cong \operatorname{Det}\left(E_{f(t)}\right) \cong H^{1}\left(E_{f(t)}, \mathcal{O}\right)^{-1} .
$$

Now a choice of isomorphism of a lattice with $[\tau, 1]$ canonically identifies the right hand side of (4.8) with $\mathbb{C}$. Further, we know how a choice of basis $\left[\omega_{1}, \omega_{2}\right]$ defines an element in $\mathbb{C} \cong H^{1}\left(E_{\left[\omega_{1}, \omega_{2}\right]}, \mathcal{O}\right)$ under the above identification: the element is $\omega_{2}$. But now observe that in the path $f$, the degree of the curve swept by $\omega_{2}$ with respect to $0 \in \mathbb{C}$ is -1 . Because of that, for a central extension $\operatorname{Det}^{r}$ of $\mathcal{A}, r \in \mathbb{C}, f(1) / f(2)=\mathrm{e}^{2 \pi i r}$, as claimed.

We shall next calculate $\mathbb{C}^{\times}$-central extensions of $\mathcal{C}_{\text {spin }}, \mathcal{C}_{\mathbb{Z} / 2}$. We begin by considering examples: Namely, in the case of $\mathcal{C}_{\text {spin }}$, we have the central extension

$$
P(X)=\operatorname{Det}\left(F: \Omega^{1 / 2}(X) \rightarrow \Omega_{+}^{1 / 2}(\partial X)\right)^{-1} .
$$

The target of $F$ is understood analogously as above, as the direct sum of copies of $\Omega_{+}^{1 / 2}\left(S_{A}^{1}\right)$, $\Omega_{-}^{1 / 2}\left(S_{A}^{1}\right), \Omega_{+}^{1 / 2}\left(S_{P}^{1}\right), \Omega_{-}^{1 / 2}\left(S_{A}^{1}\right)$ over outgoing (resp. incoming) and antiperiodic (resp. periodic) 
boundary components. The operator $F$ is restriction followed by boundary parametrization, and projection to the specified space. The proof of the fact that $P(X)$ is a central extension is analogous to the proof in the case of the determinant. Of course, $\operatorname{Det}(X)$ is a $\mathbb{C}^{\times}$-central extension of $\mathcal{C}_{\text {spin }}$ as well.

In the case of $\mathcal{C}_{\mathbb{Z} / 2}$, let $X$ be a $S q r t$-conformal surface with $S q r t$-structure given by a holomorphic complex line bundle $L$, and an isomorphism $L \otimes L \cong 1_{X}$. Let $\Gamma_{\partial X}(L)$ denote the space of $L^{2}$-sections of $L$ restricted on $\partial X$. Let $\Gamma_{X}(L)$ denote the space of holomorphic sections of $L$ on the interior of $X$, which extend to $L^{2}$-sections of $L_{\partial X}$. Then, if $X$ has no closed component, we can set

$$
Q(X)=\operatorname{Det}\left(F: \Gamma_{X}(L) \rightarrow \Gamma_{\partial X}(L)_{+}\right),
$$

and prove that this defines a $\mathbb{C}^{\times}$-central extension of $\mathcal{C}_{\mathbb{Z} / 2}$ similarly as above.

Now by the same argument as used in [21] in the case of Mumford's theorem, a $\mathbb{C}^{\times}$-central extension of $\mathcal{C}_{\text {spin }}$ or $\mathcal{C}_{\mathbb{Z} / 2}$ is determined by its restriction to $\mathcal{A}_{A}$ and $\mathcal{A}_{P}$. Now $\mathcal{A}_{A}$ is itself a central extension of $\mathcal{A}$ with kernel $\mathbb{Z} / 2$ and hence its $\mathbb{C}^{\times}$-central extension projects to a $\mathbb{C}^{\times}$-central extension of $\mathcal{A}$. Such central extension is, again, characterized by central charge $c \in \mathbb{C}$ and rotation number $h \in \mathbb{C} / \mathbb{Z}$. We will put $c_{A}=c / 2$ and $h_{A}=h / 2$ (to make the numbers compatible with [21]).

On the other hand, a $\mathbb{C}^{\times}$-central extension of $\mathcal{A}_{P} \cong \mathcal{A} \times \mathbb{Z} / 2$ is the same as a $\mathbb{C}^{\times}$-central extensions of $\mathcal{A} \subset \mathcal{A}_{P}$. We will denote its central charge and rotation by $c_{P}, h_{P}$.

To calculate these invariants for $P, Q$, Det, we include here Segal's calculation [21], Section 8, of central charge and rotation number of $\mathcal{F}\left(\Omega^{\alpha}\left(S^{1}\right)\right)$. Let $L_{p}$ denote $\mathrm{e}^{i p \theta} d / d \theta \in \operatorname{Vect}_{C}\left(S^{1}\right)$, and let $a_{q}=\mathrm{e}^{i q \theta} \in \Omega^{\alpha}\left(S^{1}\right)$. We have

$$
L_{p} a_{q}=i(q+\alpha p) a_{p+q} .
$$

Let $\omega=a_{m} \wedge a_{m-1} \wedge \cdots \in \mathcal{F}\left(\Omega^{\alpha}\left(S^{1}\right)\right.$. Then

$$
L_{p} \omega=i \sum_{k=0}^{p-1}(m-k+\alpha p) \omega_{k}
$$

if $p>0$, where $\omega_{k}$ is obtained from $\omega$ by replacing $a_{m-k}$ by $a_{m-k+p}$. If $p<0$, then $L_{p} \omega=0$. Hence,

$$
\begin{aligned}
{\left[L_{-p}, L_{p}\right] \omega } & =-\sum_{k=0}^{p-1}(m-k+\alpha p)(p+m-k-\alpha p) \\
& =-\frac{1}{12}(12 \alpha(1-\alpha)-2) p\left(p^{2}-1\right)+(\alpha(\alpha-1)-m(m+1)) p .
\end{aligned}
$$

Thus,

$$
c=12 \alpha(1-\alpha)-2, \quad h=\frac{1}{2}(m(m+1)-\alpha(\alpha-1)) .
$$

We see that for $D=D e t$, we have $m \in \mathbb{Z}, \alpha=0$ for both periodic and antiperiodic annuli, so

$$
\begin{aligned}
& c_{A}(\text { Det })=c_{P}(\text { Det })=-2, \\
& h_{A}(\text { Det })=h_{P}(\text { Det })=0 .
\end{aligned}
$$


For $D=P$, we have, in the antiperiodic case, $\alpha=1 / 2, m \in 1 / 2+\mathbb{Z}$ (but note that $h_{A} \in \mathbb{C} / \frac{1}{2} \mathbb{Z}$ ). In the periodic case, $\alpha=1 / 2, m \in \mathbb{Z}$. We conclude that

$$
\begin{aligned}
& c_{A}(P)=c_{P}(P)=1, \\
& h_{A}(P)=0, \quad h_{P}(P)=\frac{1}{8} .
\end{aligned}
$$

Thus, $P$ and Det generate a subgroup isomorphic to $\mathbb{Z} \oplus \mathbb{Z} / 4 \mathbb{Z}$ of the group of $\mathbb{C}^{\times}$-central extensions of $\mathcal{C}_{\text {spin }}$ (the group structure is the ten product).

For $Q$, the periodic case is the same for Det (since we have $\mathcal{C} \subset \mathcal{C}_{\mathbb{Z} / 2}$ ). In the antiperiodic case, $\alpha=0, m \in \frac{1}{2}+\mathbb{Z}$, so we have

$$
\begin{aligned}
& c_{A}(Q)=c_{P}(Q)=-2, \\
& h_{A}(Q)=-\frac{1}{8} \in \mathbb{C} / \frac{1}{2} \mathbb{Z}, \quad h_{P}(Q)=0 .
\end{aligned}
$$

We conclude that $Q$ and Det generate a subgroup of $\mathcal{C}_{\text {spin }}$ isomorphic to $\mathbb{Z} \oplus \mathbb{Z} / 4 \mathbb{Z}$.

Remark. - We need to observe that the $\mathcal{C}^{\times}$-central extensions Det, $P, Q$ extend to the corresponding coarser moduli spaces $\mathcal{C}_{\text {spin }}^{c}, \mathcal{C}_{\mathbb{Z} / 2}^{c}$. This is obvious for Det, which does not depend on Spin-structure (resp. Sqrt-structure).

The cases of $P, Q$ are analogous to each other. We shall discuss, say, $P$. To extend $P$ to $\mathcal{C}_{\text {spin }}^{c}$, we must specify $\mathbb{Z} / 2$-actions on the Hilbert spaces $H_{A}=\mathcal{F}\left(\Omega^{1 / 2}\left(S_{A}^{1}\right)\right), H_{P}=\mathcal{F}\left(\Omega^{1 / 2}\left(S_{P}^{1}\right)\right)$ corresponding to spin reversal, which intertwine in the appropriate way with the Spin-CFT structure on $\mathcal{F}\left(\Omega^{1 / 2}\right)$. This action is a restriction of an action of the appropriate $\mathbb{C}^{\times}$-central extension of the loop group $L S^{1}$. The intertwining of this action with Virasoro action is described in [16], while the extension of this structure to CFT is usually referred to as currents.

Very briefly, this action is induced from the action of $L S^{1}$ on the corresponding polarized Hilbert space: For $\mathbb{Z} / 2 \subset L S^{1}$ (constant -1 ), the action on polarized Hilbert space is by -1 . To lift this action to Fock space, we need a canonical element of the determinant line of its generator, or, in other words, a canonical element of $P_{L}$. However, note that a canonical element of $D_{L}$ for any $\mathbb{C}^{\times}$-central extension of $\mathcal{C}_{\text {spin }}$ is specified by the iso (4.30) below.

THEOREM 4.15. - The groups of equivalence classes of $\mathbb{C}^{\times}$-central extensions of $\mathcal{C}_{\text {spin }}$, $\mathcal{C}_{\text {spin }}^{c}$ are isomorphic to $\mathbb{Z} \oplus \mathbb{Z} / 4 \mathbb{Z}$, generated by $P$, Det. The groups of equivalence classes of $\mathbb{C}^{\times}$-central extensions of $\mathcal{C}_{\mathbb{Z} / 2}^{c}$, and of possible values of central charges and rotation numbers of $\mathbb{C}^{\times}$-central extensions of $\mathcal{C}_{\mathbb{Z} / 2}$ are isomorphic to $\mathbb{Z} \oplus \mathbb{Z} / 4 \mathbb{Z}$, generated by the central extensions $Q$, Det.

In view of Lemma 4.3, it suffices to study the restriction of the $\mathcal{C}^{\times}$-central extension to the semigroups of annuli.

This task will be divided into several lemmas.

Lemma 4.16. $-h_{A}=0$ for $\mathbb{C}^{\times}$-central extensions of $\mathcal{C}_{\text {spin }}$ and $h_{P}=0$ for $\mathbb{C}^{\times}$-central extensions of $\mathcal{C}_{\mathbb{Z} / 2}$.

Proof. - Analogous as in the case of Mumford's theorem (see (4.4)). The crucial point is that in $\mathcal{C}_{\text {spin }}$, the boundary of the unit disk is antiperiodic, while in $\mathcal{C}_{\mathbb{Z} / 2}$, it is periodic.

LEMMA 4.17. - For a $\mathbb{C}^{\times}$-central extension of $\mathcal{C}_{\text {spin }}$ or $\mathcal{C}_{\mathbb{Z} / 2}, c_{A}=c_{P}$. 
Proof. - In the case of $\mathcal{C}_{\text {spin }}$, there exists a pair of pants $X$ with two periodic and one antiperiodic boundary components. Since the tangent space to $\mathcal{C}_{\text {spin }}$ at $X$ is $\operatorname{Vect} t_{\mathbb{C}}(\partial X) / \operatorname{Vect}(X)$ $(\operatorname{Vect}(X)$ denotes holomorphic vector fields on $X$ ), we see that sewing an antiperiodic annulus to $X$ is equivalent to sewing two periodic annuli to the periodic boundary components of $X$. Passing to the Lie algebras of $\mathcal{A}_{P}, \mathcal{A}_{A}$, we see that $c_{A}$ is a linear function of $c_{P}$ (independent of the choice of central extension). Thus, we are done since our statement is true for the determinant. In the case of $\mathcal{C}_{\mathbb{Z} / 2}$, the argument is analogous, switching the roles of $A$ and $P$.

Note that since $\mathcal{C} \subset \mathcal{C}_{\mathbb{Z} / 2}$, we see that in the $\mathcal{C}_{\mathbb{Z} / 2}$-case, we have $2 \mid c$.

LEMMA 4.18. - If D is a central extension of $\mathcal{C}_{\text {spin }}\left(\right.$ resp. $\left.\mathcal{C}_{\mathbb{Z} / 2}\right)$ such that $c=0$, then $4 h_{P}=0$ (resp. $\left.4 h_{A}=0\right)$.

Proof. - In both cases of $\mathcal{C}_{\text {spin }}$ and $\mathcal{C}_{\mathbb{Z} / 2}$, if $c=0$, the method of [21], Chapter 5, gives a canonical flat connection on the bundle $D$ over the subspace of the moduli space $\mathcal{C}_{\text {spin }}$ consisting of surfaces without closed components. Thus, we have representations $\varrho_{X}: \pi_{1}\left(\mathcal{C}_{\text {spin }}, X\right) \rightarrow \mathbb{C}^{\times}$, if $X$ has no closed boundary components.

Now consider the case of $\mathcal{C}_{\text {spin }}$. Consider the curve $E_{i}^{\prime}$ as above, but with non-trivial Spin-structure given by the holomorphic line bundle on $E_{i}$ with divisor $[(\tau+1) / 2]-[0]$. Consider the loop $g_{1}$ in $\mathcal{C}_{\text {spin }}$ which transforms $E_{i}^{\prime}$ into $E_{\tau}^{\prime}$ where

$$
\tau=\frac{(1-t) i-t}{t i+(1+t)}, \quad t \in[0,1]
$$

and the loop $g_{2}$ in $\mathcal{C}_{\text {spin }}$ given by

$$
\tau=\frac{i}{2 t i+1}, \quad t \in[0,1]
$$

Then $g_{1}$ and $g_{2}$ are Dehn twists, $g_{1}$ with respect to cutting along a periodic simple curve, $g_{2}$ with respect to cutting along the double of an antiperiodic simple curve. Moreover, $g_{2} g_{1}$ is easily seen to be homotopic to the loop in $\mathcal{C}_{\text {spin }}$ at $E_{i}^{\prime}$ given by rotating the boundary parametrization $90^{\circ}$ counterclockwise. Therefore, since $\varrho\left(g_{2}\right)=0$,

$$
\varrho\left(g_{1}^{4}\right)=\varrho(\gamma)
$$

where $\gamma$ is a loop obtained by rotating the boundary parametrization $360^{\circ}$ counterclockwise.

This is the same as the map

$$
\mu^{\prime}: D_{E_{i}^{\prime}} \rightarrow D_{E_{i}^{\prime}}
$$

induced by reversal of spin. Sewing on a standard disk to the boundary of $E_{i}^{\prime}$, we see that this is equivalent to considering the map

$$
\mu: D_{E_{i}} \rightarrow D_{E_{i}}
$$

given by reversal of spin. But now cut $E_{i}$ along the simple antiperiodic curve $[i, i+1]$. The resulting surface is of the form $A_{q} \in \mathcal{A}_{A}\left(q=\mathrm{e}^{(2 \pi i) i}\right)$. Note that (4.19) has the same sign as the spin-reversing automorphism

$$
D_{A_{q}} \rightarrow D_{A_{q}}
$$

which is trivial since $D$ restricts to a trivial $\mathbb{C}^{\times}$-central extension of $\mathcal{A}_{A}$. Thus, $\varrho\left(g_{1}^{4}\right)=1$, as claimed. 
The case of $\mathcal{C}_{\mathbb{Z} / 2}$ is actually simpler: we still use the loops $g_{1}, g_{2}$, but now we consider the bundle on $E_{i}^{\prime}$ as a $S q r t$-structure. Then $g_{1}$ is still Dehn twist with respect to a periodic loop, and $g_{2}$ with respect to an antiperiodic loop. Thus, $\left(g_{2} g_{1}\right)^{4}$ is homotopic to rotating the boundary parametrization of $E_{i}^{\prime} 360^{\circ}$ counterclockwise. This is 1 , since the boundary is periodic and $D \mid \mathcal{A}_{P}$ is trivial when $c=0$. Similarly, $\varrho\left(g_{1}\right)=1$. We conclude that

$$
\varrho\left(g_{2}\right)^{4}=1 .
$$

Hence, $8 h_{A} \in \mathbb{Z}$, and $4 h_{A} \in \frac{1}{2} \mathbb{Z}$, as claimed.

Note that in the case of $\mathcal{C}_{\mathbb{Z} / 2}$, the proof of Theorem 4.15 is complete. From now on, we shall specialize to the case of $\mathcal{C}_{\text {spin }}$.

\section{LEMMA 4.20. - For a holomorphic central extension of $\mathcal{C}_{\text {spin }}, c \in \mathbb{Z}$.}

Proof. - We shall consider the loops $g_{1}, g_{2}$ as in the proof of Lemma 4.18 in $\mathcal{C}_{\text {spin }}$ at $E_{i}^{\prime}$, with the Spin-structure induced by the bundle $[(i+1) / 2]-[0]$ on $E_{i}$. Then $\left(g_{2} g_{1}\right)^{8}$ is equal to a loop $f$ in $\mathcal{C}_{\text {spin }}$ obtained by varying the boundary component of $E_{i}^{\prime}$ (and its parametrization), i.e. to a loop which is an integral of a path in $\operatorname{Vect}_{\mathbb{C}}\left(S^{1}\right)$. Now fixing lifts of $\hat{g}_{1}, \hat{g}_{2}$ to the line bundle on $\mathcal{C}_{\text {spin }}$ given by the $\mathbb{C}^{\times}$-central extension, $f$ will lift to a path $\hat{f}$ in the line bundle, and hence we can measure the ratio of its beginning- and end-points, which would be a certain number in $\mathbb{C}^{\times}$. Denote the logarithm of this number, divided by $2 \pi i$, by $q=q(f)$. Now choosing a representative of $h_{P}$ modulo $\mathbb{Z}$, we can pick canonical lifts $\hat{g}_{1}, \hat{g}_{2}$ (the canonical representatives of $L_{0}$ in the central extension of $\operatorname{Vect} t_{\mathbb{C}}^{S^{1}}$ ). This gives

$$
q \equiv 8 h \quad \bmod \mathbb{Z},
$$

since, using analogous notation for $g_{i}$ as for $f$,

$$
q\left(g_{1}\right)=h, \quad q\left(g_{2}\right)=0 .
$$

Also, by the above analysis using a pair of pants with two periodic and one antiperiodic boundary, the number $q$ is defined in Lie algebra terms only, and hence does not depend on Spin-structure. But using the case of the determinant, we then see that

$$
q=c .
$$

But replacing the Spin-structure on $E_{i}^{\prime}$ by the trivial Spin-structure, we then have

$$
q\left(g_{1}\right)=q\left(g_{2}\right)=h,
$$

and hence

$$
q \equiv 24 h \quad \bmod \mathbb{Z} .
$$

Comparing (4.21) and (4.23), we then get

$$
16 h \in \mathbb{Z},
$$

and comparing this with (4.22) and (4.21), we get

$$
c \in \frac{1}{2} \mathbb{Z} .
$$


But now if, in fact, we had $c \in \frac{1}{2}+\mathbb{Z}$, then, by (4.21),

$$
h \in \frac{1}{16}+\frac{1}{8} \mathbb{Z} .
$$

To see this scenario is impossible, consider a periodic annulus $A$ with one inbound and one outbound boundary component of the same spin. Let $L$ be the infinitesimal periodic annulus representing spin reversal ( $L$ can be replaced by an annulus of finite thickness for models where the existence of infinitesimal spin-reversion annuli is not assumed). Now spin reversal on $A$ can be interpreted as the isomorphism

$$
A \stackrel{\sigma}{\longrightarrow}(L \circ A \circ L)
$$

(Here $\circ$ denotes sewing regarded as composition of annuli.) An automorphism of a line is multiplication by a non-zero complex number. We see by definition that the number associated with the automorphism of $D_{E}$ corresponding to spin reversal on $E$ is equal to the number associated with the automorphism

$$
\begin{array}{r}
D_{A} \stackrel{\sigma}{\longrightarrow} D_{L \circ A \circ L} \stackrel{\cong}{\longleftarrow} D_{L} \otimes D_{A} \otimes D_{L} \stackrel{T \otimes 1}{\cong} D_{A} \otimes D_{L} \otimes D_{L} \\
\cong \mid 1 \otimes T \\
\vee \\
D_{A} \otimes D_{L} \otimes D_{L} \\
|\downarrow| 1 \otimes \text { sewing } \\
D_{A} .
\end{array}
$$

On the other hand, similarly, the number associated with the automorphism on $D$ associated with spin reversal on the elliptic curve $E^{\prime}$ obtained by sewing together the boundary components of $L \circ A$ is

$$
D_{L} \otimes D_{A} \stackrel{1 \otimes \sigma}{\longrightarrow} D_{L} \otimes D_{L} \otimes D_{A} \otimes D_{L} \stackrel{\text { sew } \otimes 1 \otimes 1}{\longrightarrow} D_{A} \otimes D_{L} \stackrel{T}{\longrightarrow} D_{L} \otimes D_{A} .
$$

Since $A$ has degree 0, the ratio between the numbers associated with (4.27) and (4.28) is the number associated with

$$
D_{L} \otimes D_{L} \stackrel{T}{\longrightarrow} D_{L} \otimes D_{L}
$$

which is $(-1)^{\operatorname{deg}(L)}$. Thus, we see that

$$
\operatorname{deg}(L) \equiv 1 \quad \bmod 2
$$

However, now consider the periodic (standard) annulus $B$ with two outbound boundary components. Then if we select boundary parametrizations appropriately, there is an isomorphism in $\mathcal{C}_{\text {spin }}$

$$
B \cong L \circ B
$$

switching boundary components, which contradicts (4.29).

This concludes the proof of Theorem 4.15. We saw that cutting along a periodic curve played an essential role in excluding $c=1 / 2$ in the above lemma. 


\section{The Pfaffian central extension}

We will now show that the conclusion of Theorem 4.15 changes if we change the axioms in a suitable way.

Consider the case of $X$ closed first. Considering $\Omega^{1 / 2}$ as a sheaf, note that we have a pairing

$$
H^{0}\left(X, \Omega^{1 / 2}\right) \otimes H^{1}\left(X, \Omega^{1 / 2}\right) \rightarrow \mathbb{C}
$$

given by

$$
\omega \otimes \xi \rightarrow \int_{X} \omega \xi .
$$

The pairing (5.1) is non-degenerate, and hence we have a natural isomorphism

$$
H^{0}\left(X, \Omega^{1 / 2}\right) \cong\left(H^{1}\left(X, \Omega^{1 / 2}\right)\right)^{\vee}
$$

where $(?)^{\vee}$ denotes the dual. In view of (2.19), it is therefore natural to define

$$
\operatorname{Pf}(X):=\operatorname{Det}\left(H^{0}\left(X, \Omega^{1 / 2}\right)\right) .
$$

Now suppose $X$ is a connected Spin-surface with non-empty boundary. Recall that, for such $X, \Omega^{1 / 2} \partial X$ denotes the space of complex-valued $L^{2}-1 / 2$-forms on $\partial X$ with values in $\mathbb{C}$. Recall also that $\Omega^{1 / 2} X$ denotes the space of $L^{2}-1 / 2$-forms on $\partial X$ with an extension to holomorphic $1 / 2$-forms in the interior of $X$. As usual, $\Omega^{1 / 2} \partial X$ is more precisely viewed as $\Omega^{1 / 2}\left(\amalg S_{A}^{1} \amalg \amalg S_{P}^{1}\right)$ where the disjoint sum is over the boundary components of $X$. As such, the Spin-structure on $\partial X$ is canonically a complexification of a real bundle, namely the bundle of real $1 / 2$-forms on $\partial X$, which gives it a real structure (a complex conjugation map). This is, however, slightly different from the real structure we will need to use. To this end, consider the symmetric bilinear form $B$ on $\Omega^{1 / 2} \partial X$ given by

$$
B(\omega, \eta)=\int_{\partial X} \omega \eta .
$$

The integration is in the direction of the parametrization of outbound boundary components, but against the direction of the parametrization of inbound boundary components (to make holomorphic $1 / 2$-forms on $X$ isotropic). Then we need a different real structure on $\Omega^{1 / 2}(\partial X)$, such that $B$ is positive definite on $\left(\Omega^{1 / 2}(\partial X)\right)_{\mathbb{R}}$. Such real structure is obtained by multiplying the old complex conjugation operator by -1 on inbound boundary components, which is equivalent to multiplying the space of real $1 / 2$-forms by $i$ on inbound boundary components. We will use this new real structure from this point on. Let $\bar{?}$ denote complex conjugation with respect to the new real structure.

Now to define Pfaffian for Spin-surfaces $X$ with boundary, we must pick a complex structure on $\Omega^{1 / 2}(\partial X)_{\mathbb{R}}$ (by which we mean real $1 / 2$-forms, with respect to the form $B$, on a disjoint union of copies of $S_{A}^{1}, S_{P}^{1}$ corresponding to boundary components of $X$ ).

There is a canonical complex structure on $\Omega^{1 / 2}\left(S_{A}^{1}\right)_{\mathbb{R}}$, where the isotropic subspaces of $\Omega^{1 / 2}\left(S_{A}^{1}\right)_{\mathbb{C}}$ are the spaces of holomorphic and antiholomorphic $1 / 2$-forms on the standard disk.

However, the situation is different for $\Omega^{1 / 2}\left(S_{P}^{1}\right)$. There is no complex structure on $\Omega^{1 / 2}\left(S_{P}^{1}\right)$ compatible with polarization! 
In general, if $H_{\mathbb{R}}$ is a real Hilbert space with inner product $B$, then call a polarization of $H$ even-dimensional if there is a complex structure on $H_{\mathbb{R}}$ which is compatible with polarization. Otherwise, call $H_{\mathbb{R}}$ odd-dimensional.

The invariant distinguishing even- and odd-dimensional polarized real Hilbert spaces is given as follows: It is known ([16], Chapter 12) that the restricted orthogonal group satisfies

$$
O_{\text {res }} H_{\mathbb{R}} \simeq O / U
$$

In particular, $O_{r e s} H_{\mathbb{R}}$ has two connected components, which gives a canonical homomorphism

$$
h: O_{\text {res }} H_{\mathbb{R}} \rightarrow \mathbb{Z} / 2 .
$$

We say that $h$ is even- (resp. odd-) dimensional if $h(-1)=1$ (resp. $h(-1)=-1$ ). It can be shown that $\Omega^{1 / 2}\left(S_{P}^{1}\right)$ is odd-dimensional.

The space $\Omega^{1 / 2} \partial X$ for $X \in \mathcal{C}_{\text {spin }}$, however, is even-dimensional. We can pick a complex structure on $\left(\Omega^{1 / 2} \partial X\right)_{\mathbb{R}}$ compatible with polarization. But since there is no such canonical pick, the only way I know of defining the central extension $\sqrt{P}$ for surfaces with periodic boundary components is to make the complex structure a part of the moduli space:

Let $\mathcal{C}_{\text {spin }}^{\prime}$ be the moduli space of Spin-surfaces $X$ together with a specified complex structure, compatible with polarization, on the space of real $1 / 2$-forms on the boundary $\Omega^{1 / 2}(\partial X)_{\mathbb{R}}$ (with respect to the symmetric bilinear form $B$ ). From a stack point of view, if $T$ is a Teichmüller space for a connected component of $\mathcal{C}_{\text {spin }}$, then the corresponding Teichmüller space $T^{\prime}$ of $\mathcal{C}_{\text {spin }}^{\prime}$ is isomorphic to $T \times O_{\text {res }} / U_{\text {res }}$. The modular group $G$ is the same for $\mathcal{C}_{\text {spin }}$ and $\mathcal{C}_{\text {spin }}^{\prime}$, and its action commutes with the canonical projection $T^{\prime} \rightarrow T$.

To define sewing on $\mathcal{C}_{\text {spin }}^{\prime}$, we shall consider a certain class of distinguished complex structures $J_{0}$ on $\Omega^{1 / 2}\left(C^{\text {in }} \amalg C^{\text {out }}\right)_{\mathbb{R}}$ where $C$ stands for $S_{A}^{1}$ or $S_{P}^{1}$. Denote by $P$ the operator

$$
\Omega^{1 / 2}\left(C^{\text {in }} \amalg C^{\text {out }}\right)_{\mathbb{C}} \cong \Omega^{1 / 2}\left(C^{\text {in }}\right)_{\mathbb{C}} \oplus \Omega^{1 / 2}\left(C^{\text {out }}\right)_{\mathbb{C}} \stackrel{\nabla}{\longrightarrow} \Omega^{1 / 2}(C)_{\mathbb{C}}
$$

where $\nabla(x, y)=y-x$. Then $J_{0}$ is distinguished if

$$
P: \Omega^{1 / 2}\left(C^{\text {in }} \amalg C^{\text {out }}\right)_{\mathbb{C},+} \rightarrow \Omega^{1 / 2}(C)_{\mathbb{C}}
$$

is an isomorphism, where $H_{\mathbb{C},+}$ is, as usual, the $i$-eigenspace of a complex structure on $H_{\mathbb{R}}$.

Now a sewing map corresponding to $C^{\text {in }}, C^{\text {out }}$ will be defined for surfaces $X$ for which the complex structure on the space of real $1 / 2$-forms of $\partial X$ is of the form $J_{0} \oplus J$, where $J_{0}$ is a distinguished complex structure on $\Omega^{1 / 2}\left(C^{\text {in }} \amalg C^{\text {out }}\right)_{\mathbb{R}}$ compatible with polarization, and $J$ is some complex structure on $\Omega^{1 / 2}\left(\partial X-\left(C^{\text {in }} \amalg C^{\text {out }}\right)\right)_{\mathbb{R}}$, also compatible with polarization. We will then also say that the complex structure $J_{0} \oplus J$ is distinguished, with respect to the pair of boundary components $C^{\text {in }}, C^{\text {out }}$.

From a stack point of view, this corresponds to defining the sewing map on the corresponding substack of a connected component of $\mathbb{C}_{\text {spin }}^{\prime}$. Diagrams (2.5)-(2.8) are interpreted in an analogous way as in the previous cases, thus giving a notion of holomorphic $\mathbb{C}^{\times}$-central extension on $\mathcal{C}_{\text {spin }}^{\prime}$.

When considering this analogy, however, we must keep in mind the substantial restrictions imposed by the fact that sewing is one defined on distinguished complex structures: For example, for two pairs $C_{i}^{\text {in }}, C_{i}^{\text {out }}, i=1,2$, of disjoint boundary components where $C_{i}^{\text {in }}, C_{i}^{\text {out }}$ for the same $i$ are both periodic or antiperiodic, the diagram expressing independence of sewing along 
$C_{i}^{\text {in }}, C_{i}^{\text {out }}$ on the order of sewing ( $i=1,2$ vs. $i=2,1$ ) has as its source the stack of Spin-surfaces with the boundary components $C_{i}^{\text {in }}, C_{i}^{\text {out }}$ and three complex structures on

$$
\begin{gathered}
\Omega^{1 / 2}\left(C_{1}^{\text {in }} \amalg C_{1}^{\text {out }}\right)_{\mathbb{R}}, \\
\Omega^{1 / 2}\left(C_{2}^{\text {in }} \amalg C_{2}^{\text {out }}\right)_{\mathbb{R}}, \\
\Omega^{1 / 2}\left(\partial X-\coprod_{i=1,2}\left(C_{i}^{\text {in }} \amalg C_{i}^{\text {out }}\right)\right)_{\mathbb{R}}
\end{gathered}
$$

where the first two are distinguished.

Remark. - It is easy to see that there exist distinguished complex structures on $\Omega^{1 / 2}\left(C^{\text {in }} \amalg C^{\text {out }}\right)_{\mathbb{R}}$ compatible with polarization. For example, take a standard annulus $A$ with boundary $C^{\text {in }} \amalg C^{\text {out }}$ with opposite spin, and take the complex structure determined by the isotropic subspaces $\Omega^{1 / 2}(A), \overline{\Omega^{1 / 2}(A)}$ of holomorphic and antiholomorphic forms on $A$. This is a complex structure, because the elliptic curve $E$ obtained from $A$ by sewing $C^{\text {in }}$ and $C^{\text {out }}$ has non-trivial Spin-structure: then, since $\Omega^{1 / 2}$ is a line bundle of degree 0 , and is non-trivial, we have $H^{*}\left(E, \Omega^{1 / 2}\right)=0$. Now recall that $H^{*}\left(E, \Omega^{1 / 2}\right)$ is the cohomology of the complex

$$
\Omega^{1 / 2}(A) \oplus \overline{\Omega^{1 / 2}(A)} \rightarrow \Omega^{1 / 2}\left(C^{\text {in }} \amalg C^{\text {out }}\right)_{\mathbb{C}},
$$

so in this case the map (5.5) is an iso, as required.

THEOREM 5.6. - There exists a $\mathbb{C}^{\times}$-central extension $\sqrt{P}$ of $\mathcal{C}_{\text {spin }}^{\prime}$, i.e. a central extension whose square is the pullback of $P$ to $\mathcal{C}_{\text {spin }}^{\prime}$.

It is helpful to introduce the notion of Pfaffians of operators: first, given a Fredholm operator $F: U \rightarrow V$ together with an explicit isomorphism

$$
\operatorname{Ker}(F) \cong \operatorname{Coker}(F)^{\vee}
$$

we can define the Pfaffian of $F$ as

$$
\operatorname{Pf}(F):=\operatorname{Det}(\operatorname{Coker}(F)) \cong(\operatorname{Det}(\operatorname{Ker}(F)))^{-1} .
$$

However, we shall need a "continuity property" similar to the discussion we had in the case of the determinant line. To this end, we prove the following

Claim 5.9. - Assume Coker $(F)$ is even-dimensional. Then there exist operators $S: V^{*} \rightarrow V$, $T: U \rightarrow V^{*}$ such that $F+S T$ is invertible, $S, T$ are Hilbert-Schmidt, $S$ is skew and

$$
\operatorname{Im}\left(\begin{array}{c}
F \\
T
\end{array}\right) \subset V \oplus V^{*}
$$

is isotropic with respect to the canonical symmetric bilinear form on $V \oplus V^{*}$ (where $V$ is isotropic, and for $\left.v \in V, w \in V^{*}, B(v, w)=w(v)\right)$.

Proof. - Since $F$ can be replaced by $F A$ where $A$ is any invertible operator, we may assume that $F$ is a projection. Let its 0 - and 1-eigenspaces be $K, W$, respectively. Then

$$
K=\operatorname{Ker}(F)=\operatorname{Coker}(F),
$$


so (5.7) gives a real structure on $K$. Denoting by $K_{\mathbb{R}}$ the real subspace, let $J$ be any complex structure on $K_{\mathbb{R}}$. Then we can put $S=J, T=-J$ (extended by 0 on $W$ ).

For us, this motivates defining the Pfaffian from yet a different set of data, namely an inclusion

$$
\left(\begin{array}{l}
F \\
T
\end{array}\right): U \subset V \oplus V^{*}
$$

whose image is isotropic, $T$ is Hilbert-Schmidt and there is a skew Hilbert-Schmidt operator $S: V^{*} \rightarrow V$ such that $F+S T$ is invertible. (This requires $\operatorname{Ker}(F)$ to be even-dimensional.) Then we can define the Pfaffian as the line generated by $F+S T$. Note that if we replace $S$ by another skew Hilbert-Schmidt operator $S^{\prime}$ such that $F+S^{\prime} T$ is invertible, then we get a canonical isomorphism of Pfaffian lines, as

$$
\left(F+S^{\prime} T\right)^{-1}(F+S T)=1+\left(S-S^{\prime}\right) T\left(F+S^{\prime} T\right)^{-1},
$$

while $T\left(F+S^{\prime} T\right)^{-1}$ is skew. Thus, the right hand side of (5.10) is of the form $1+A B$ with $A, B$ Hilbert-Schmidt and skew, and such operators have a canonical Pfaffian (see [16], Chapter 12).

Similarly as in the case of the determinant, another way to look at this discussion is not as a definition, but as a "continuity statement", specifying an isomorphism between the Pfaffians of the operators $F, F+S T$.

Following this terminology, we shall denote the desired $\mathbb{C}^{\times}$-central extensions $\sqrt{P}$ by $P f$, although, in comparison with our previous notation, $P f^{2}$ is then not isomorphic to $\operatorname{Det}$ (since by Det we denoted the determinant of holomorphic functions, not $1 / 2$-forms). To define $\operatorname{Pf}(X)$, note that our complex structure on $\Omega^{1 / 2}(\partial X)_{\mathbb{R}}$ gives a decomposition $\left(\Omega^{1 / 2} \partial X\right)_{\mathbb{C}}=V \oplus \bar{V}$ for an isotropic space $V$ (the $i$-eigenspace of the complex structure). We can then define $\operatorname{Pf}(X)$ as the Pfaffian of the inclusion

$$
\left(\begin{array}{c}
F \\
T
\end{array}\right): \Omega^{1 / 2}(X) \rightarrow V \oplus \bar{V}
$$

To be able to choose $S$ skew Hilbert-Schmidt so that $F+S T$ is invertible, $\operatorname{Ker}(F)$ must be even-dimensional. If it is not, however, we can change the complex structure on $\Omega^{1 / 2}(\partial X)_{\mathbb{R}}$ to make the kernel even-dimensional, and then divide by the Pfaffian corresponding to change of complex structure, which is defined in [16], Chapter 12.

Another way to say this is that the $B$-isotropic space

$$
\Omega^{1 / 2}(X) \subset \Omega^{1 / 2}(\partial X)
$$

(assuming $X$ has no closed connected components) has relative dimension 0 with respect to the spacce $V$ (with $V \oplus \bar{V}=\Omega^{1 / 2}(\partial X)$ ). On the other hand,

$$
\Omega^{1 / 2}(X) \cap \overline{\Omega^{1 / 2}(X)}=0 .
$$

To see this, note that an element $\omega \in \Omega^{1 / 2}(X) \cap \overline{\Omega^{1 / 2}(X)}$ would be real, i.e. would satisfy $B(\omega, \omega)>0$ (if $\omega \neq 0$ ), contradicting the fact that $\Omega^{1 / 2}(X)$ is isotropic.

Now (5.12) and the fact that $\Omega^{1 / 2}(X)$ has 0 relative dimension with respect to $V$ implies that

$$
\Omega^{1 / 2}(X) \oplus \overline{\Omega^{1 / 2}(X)}=\Omega^{1 / 2}(\partial X),
$$

and hence $P f_{V}\left(\Omega^{1 / 2} X\right)$ is defined in [16], Chapter 12 . 
To define a sewing isomorphism along two boundary components $C^{\text {in }}$ and $C^{\text {out }}$ of $X$ with distinguished complex structure $J_{0} \oplus J$ on $\Omega^{2}(\partial X)_{\mathbb{R}}$ as above, note that there is another, canonical, complex structure $J_{0}^{\prime}$ on

$$
\Omega^{1 / 2}\left(C^{\text {in }} \amalg C^{\text {out }}\right)_{\mathbb{R}}=\Omega^{1 / 2}\left(C^{\text {in }}\right)_{\mathbb{R}} \oplus \Omega^{1 / 2}\left(C^{\text {out }}\right)_{\mathbb{R}} .
$$

To this end, recall first that the subscript $\mathbb{R}$ refers to the real structure corresponding to $B$, which multiplies the obvious real structure (square root of tangent vector to the boundary in the direction of the parametrization) by $i$ on inbound boundary components. Thus, indentifying $C^{\text {in }}$ and $C^{\text {out }}$ via the canonical iso, a complex structure on (5.13) is given by the matrix

$$
\left(\begin{array}{cc}
0 & -i \\
-i & 0
\end{array}\right)
$$

Observe that the $i$-eigenspace then consists of vectors of the form

$$
\left(\begin{array}{c}
-v \\
v
\end{array}\right), \quad v \in \Omega^{1 / 2}(C)_{\mathbb{C}}
$$

and the $-i$-eigenspace consists of vectors of the form

$$
\left(\begin{array}{l}
v \\
v
\end{array}\right), \quad v \in \Omega^{1 / 2}(C)_{\mathbb{C}}
$$

Therefore, projection onto the $i$-eigenspace is the map

$$
\left(\begin{array}{c}
u \\
v
\end{array}\right) \mapsto \frac{u-v}{2}
$$

By definition, $\operatorname{Pf}(\check{X})$ is canonically isomorphic to the Pfaffian of an operator

$$
\begin{gathered}
\Omega^{1 / 2}(X) \longrightarrow \Omega^{1 / 2}\left(C^{\text {in }} \amalg C^{\text {out }}\right)_{\mathbb{C}} \oplus \Omega^{1 / 2}\left((\partial X)-\left(C^{\text {in }} \amalg C^{\text {out }}\right)\right)_{\mathbb{C}} \\
\downarrow \\
\downarrow \\
G_{1} \oplus G_{2} \\
\Omega^{1 / 2}(C)_{\mathbb{C}} \oplus \Omega^{1 / 2}\left((\partial X)-\left(C^{\text {in }} \amalg C^{\text {out }}\right)\right)_{\mathbb{C},+}
\end{gathered}
$$

where the first operator is restriction, the operator $G_{2}$ is projection to $i$-eigenspace of the complex structure $J$, and $G_{1}$ is the restriction of the operator (5.15).

In other words, the Pfaffian of $\check{X}$ is canonically isomorphic to the Pfaffian of $X$ with respect to the complex structure $J_{0}^{\prime} \oplus J$ on $\Omega^{1 / 2}(\partial X)_{\mathbb{R}}$.

Now the condition that $J_{0}$ be distinguished says that the projection operator from the $i$-eigenspace of $J_{0}$ to the $i$-eigenspace of $J_{o}^{\prime}$ is an isomorphism. Following [16], Chapter 12, this gives a canonical trivialisation of the Pfaffian of the transition matrix from $J_{0}$ to $J_{0}^{\prime}$.

There is a slight problem with this argument, due to the fact that, as the reader may have realized, the canonical complex structure $J_{0}^{\prime}$ is not compatible with polarization. (In fact, note that there is a similar issue in the case of the determinant, if we describe the gluing isomorphism in this language.) 
Applying the methods of [16], Chapter 12, in this case will leave us with the task of showing that we have a canonical isomorphism between the Pfaffians of Fredholm operators of the form

$$
F, \quad F(I-S Q)
$$

where $S, Q$ are skew and $S Q$ is trace class (but $S, Q$ are not necessarily Hilbert-Schmidt). In other words, we must show that an operator of the form

$$
I-S Q
$$

for $S, Q$ skew and $S Q$ trace class, has a canonical Pfaffian (a number). To this end, note that the formula

$$
\operatorname{Pf}(I-S T)=\sum_{\sigma} \operatorname{Pf}\left(S_{\sigma}\right) \operatorname{Pf}\left(Q_{\sigma}\right)
$$

of [16], Chapter 12, makes sense in the case when $S, Q$ are skew and $S Q$ is trace class. To see this, note that the formula is proven by taking limit over skew submatrices of $S, Q$ which have only finitely many non-zero entries: for such matrices, the formula gives a square root of the determinant. Thus, (5.16) must converge, since $\operatorname{det}(I-S T)$ converges and its two square roots form a discrete set.

The situation simplifies if we restrict attention to the submoduli space $\mathcal{C}_{\text {spin }}^{A}$ of $\mathcal{C}_{\text {spin }}$ consisting of Spin-surfaces whose each boundary component is antiperiodic.

THEOREM 5.17. - There exists a $\mathbb{C}^{\times}$-central extension $\sqrt{P}$ on $\mathcal{C}_{\text {spin }}^{A}$ (i.e. a $\mathbb{C}^{\times}$-central extension whose square is the restriction of $P$ to $\left.\mathcal{C}_{\text {spin }}^{A}\right)$.

Proof. - As remarked above, there is a canonical complex structure on $\Omega^{1 / 2}\left(S_{A}^{1}\right)_{\mathbb{R}}$ compatible with polarization, whose isotropic subspaces of $\Omega^{1 / 2}\left(S_{A}^{1}\right)_{\mathbb{C}}$ are the spaces of holomorphic and antiholomorphic $1 / 2$-forms on the standard disk. Moreover, if we reverse this complex structure on the inbound boundary component, we obtain a complex structure on

$$
\Omega^{1 / 2}\left(\left(S_{A}^{1}\right)^{\text {in }} \amalg\left(S_{A}^{1}\right)^{\text {out }}\right)_{\mathbb{R}}
$$

which is obviously distinguished in the sense defined above. Therefore, we have defined a canonical complex structure on $\Omega^{1 / 2}(\partial X)_{\mathbb{R}}$ for every $X \in \mathcal{C}_{\text {spin }}^{A}$ which is distinguished with respect to every pair consisting of an inbound and outbound boundary component. Further, this complex structure is compatible with sewing.

Therefore, we have constructed a map

$$
\mathcal{C}_{\text {spin }}^{A} \rightarrow \mathcal{C}_{\text {spin }}^{\prime}
$$

compatible with sewing, and our statement therefore follows from Theorem 5.6.

Both of these approaches leave open the question as to whether there is a generalization of the concept of $\mathbb{C}^{\times}$-central extension which would include the case of $\sqrt{P}$, and which would be defined on $\mathcal{C}_{\text {spin }}$ itself, without introducing additional structure. Such concept indeed exists, and is due to P. Deligne.

We need the language of super-Brauer groups due to C.T.C. Wall [23,5]. Consider, for a field $k$, the graded tensor category $s \operatorname{Vect}(k)$ of super- $k$-vector spaces, i.e. $\mathbb{Z} / 2$-graded vector spaces where the commutativity isomorphism

$$
T: V \otimes W \rightarrow W \otimes V
$$

$4^{\mathrm{e}}$ SÉRIE - TOME $36-2003-\mathrm{N}^{\circ} 1$ 
is defined by

$$
T(v \otimes w)=(-1)^{\operatorname{deg}(v) \operatorname{deg}(w)} w \otimes v
$$

for homogeneous elements $v, w$. Morphisms in $s \operatorname{Vect}(k)$ are homomorphisms preserving degree. An algebra in $s \operatorname{Vect}(k)$ (super-algebra) is then the same as a $\mathbb{Z} / 2$-graded $k$-algebra, but the notion of center is defined in the graded sense, thus giving a new notion of super-central simple algebra over $k$.

A Morita equivalence between two super-central simple algebras $D_{1} \sim D_{2}$ can be defined as an additive functor from the category of $D_{1}$-modules to the category of $D_{2}$-modules which is an equivalence of categories. Up to isomorphism, all such functors are of the form $M \otimes_{D_{1}}$ ? where $M$ is a right $D_{1}$-module whose commutant is isomorphic to $D_{2}$. The commutant of $V$ is the subalgebra of $\underline{E n d}(V)$ consisting of endomorphism commuting with the right $D_{1}$-action. $\underline{\operatorname{End}}(V)$ is $V^{*} \otimes V$, i.e. the algebra of (not necessarily graded) endomorphisms of $V$.

The super-Brauer group $s B r(k)$ then is the group of Morita equivalence classes of finite supercentral simple algebras over $k$ (with respect to the graded tensor product $\otimes$ ). For our purposes, we only need the case $k=\mathbb{C}$. The group $s B r(\mathbb{C})$ is isomorphic to $\mathbb{Z} / 2$, with generator $D_{0}$ which is of the form $\mathbb{C}($ even $) \oplus \mathbb{C} \eta($ odd $), \eta^{2}=1$. Every super-central simple algebra over $\mathbb{C}$ is isomorphic to $\underline{\operatorname{End}}(V)$ or $\underline{\operatorname{End}}(V) \otimes D_{0}$ for some $\mathbb{Z} / 2$-graded complex vector space $V$. The categories of super-modules over these algebras are easy to describe: The category of $\underline{E n d}(V)$-super-modules is equivalent to $s \operatorname{Vect}(\mathbb{C})$, the category of $\underline{\operatorname{End}}(V) \otimes D_{0}$-super-modules is equivalent to $\operatorname{Vect}(\mathbb{C})$ (the category of ungraded $\mathbb{C}$-vector spaces).

We shall need two observations: First, there is s canonical iso (hence Morita equivalence) of super-algebras

$$
D_{1} \otimes D_{2} \cong D_{2} \otimes D_{1}
$$

Second, for every super-algebra $D$, there is a super-algebra $D^{o p}$ which is $D$ with operation

$$
x \cdot{ }_{\text {op }} y=(-1)^{\operatorname{deg}(x) \operatorname{deg}(y)} y \cdot x
$$

(for $x, y$ homogeneous). We have a canonical Morita equivalence

$$
D \otimes D^{o p} \sim \mathbb{C}
$$

Now a holomorphic $s B r(\mathbb{C})-\mathbb{C}^{\times}$-central extension of $\mathcal{C}_{\text {spin }}$ consists of the following data:

(1) A $\mathbb{C}$-superalgebra $D$. Then define, for $X \in \mathcal{C}_{\text {spin }}$,

$$
D(X)=\bigotimes_{\text {out }, P} D \otimes \bigotimes_{\text {in }, P} D^{o p}
$$

where the first (resp. second) tensor product is over outbound (resp. inbound) periodic boundary components.

(2) For each $X \in \mathcal{C}_{\text {spin }}$, an irreducible $D(X)$-module $M(X)$ (more precisely described in the above stack language, i.e. in particular $M(X)$ form holomorphic Spin-bundles over the connected components of $\mathcal{C}_{\text {spin }}$ ).

(3) For $\check{X}$ obtained from $X$ by sewing, an isomorphism between

$$
M(\check{X})
$$

and the $D(\check{X})$-module corresponding to $M(X)$ by the canonical Morita equivalence $D(S) \sim D(\check{X})$ coming from (5.20). 
(4) For $X, Y \in \mathcal{C}_{\text {spin }}$, an isomorphism between $M(X) \otimes M(Y)$ and the $D(X) \otimes D(Y)$ module coming from the canonical iso $D(X) \otimes D(Y) \cong D(X \amalg Y)$.

(5) Coherence diagrams analogous to (2.5)-(2.8). In particular, the analogue of (2.8) uses the isomorphism (5.18).

THEOREM 5.21. - There exists a $s B r(\mathbb{C})-\mathbb{C}^{\times}$-central extension $\sqrt{P}$ of $\mathcal{C}_{\text {spin }}$ (i.e. such that we have an isomorphism of $s B r(\mathbb{C})-\mathbb{C}^{\times}$-central extensions $\left(\sqrt{P}{ }^{\otimes 2} \cong P\right)$ ).

Proof. - Suppose $H_{\mathbb{R}}$ is a polarized real Hilbert space, and $W_{0} \in G r_{r e s}\left(H_{\mathbb{C}}\right)$ is an isotropic space such that

$$
W_{0} \oplus \overline{W_{0}}=H_{\mathbb{C}},
$$

so $W_{0}$ is equivalent to giving a complex structure on $H_{\mathbb{R}}$ (see [16], Chapter 12). Now suppose that we have another complex structure, i.e. another isotropic space $W \in G r_{r e s}\left(H_{\mathbb{C}}\right), W \oplus \bar{W}=H_{\mathbb{C}}$, then [16], Definition 12.4.3 gives a canonical line $P f_{W_{0}}(W)$ (which we already used).

The key point of the present argument is to understand how the Pfaffian line changes if we fix an isotropic subspace $W_{0} \in G r_{r e s}\left(H_{\mathbb{C}}\right)$ not necessarily satisfying (5.22). Let $V$ be the (finitedimensional) orthogonal complement of $W_{0} \oplus \overline{W_{0}}$ in $H_{\mathbb{C}}$. We have a quadratic form on $V$ induced by the real structure on $H$.

OBSERVATION 5.23. - For a $W_{0}$ just described, $W$ as above gives a canonical irreducible module $\operatorname{Pf}_{W_{0}}(W)$ over the Clifford algebra $C(V)$.

Proof. - In the case $W=W_{0} \oplus Z$ where

$$
Z \oplus \bar{Z}=V,
$$

put $P f_{W_{0}}(W)=\Lambda(Z)$. For general $W$, let $P f_{W_{0}}(W)$ be $\Lambda(Z)$ tensored with the Pfaffian line $P f_{W_{0} \oplus Z}(W)$. The theory developed in [16], Chapter 12, shows that the Clifford modules thus defined are canonically isomorphic for different choices of $Z$.

Now we must choose (and fix) maximal isotropic subspaces $W_{0}^{A}, W_{0}^{P}$ in the restricted Grassmanians of $\Omega^{1 / 2} S_{A}^{1}, \Omega^{1 / 2} S_{P}^{1}$. We have already chosen $W_{0}^{A}$ as the space of $1 / 2$-forms extending to holomorphic $1 / 2$-forms on the unit disk. In the case of $\Omega^{1 / 2} S_{P}^{1}$, choose

$$
W_{0}^{P}=\left\langle z^{n} d u^{1 / 2} \mid n>0\right\rangle, \quad \text { where } z=\mathrm{e}^{2 \pi i u} .
$$

Then in the antiperiodic case, $V=V^{A}=0$, so the corresponding Clifford algebra is $\mathbb{C}$, and in the periodic case $V=V^{P}=\mathbb{C} \cdot d u^{1 / 2}$, so $D=C\left(V^{P}\right)$ is a super-division algebra. Note that if we reverse the sign of the symmetric bilinear form on $\Omega^{1 / 2} S_{P}^{1}$ (which happens on inbound boundary components), $W_{0}^{P}$ remains isotropic, while $D$ is replaced by $D^{O p}$.

Thus, for a Spin-surface $X$, we have a canonical isotropic subspace

$$
W_{0}(X)=\bigoplus_{A} W_{0}^{A} \oplus \bigoplus_{P} W_{0}^{P} \subset \Omega^{1 / 2}(\partial X)
$$

where the sums are over periodic (resp. antiperiodic) boundary components. Then if we denote by $V(X)$ the orthogonal complement of $W_{0}(X) \oplus \overline{W_{0}(X)}$ in $\Omega^{1 / 2}(\partial X)_{\mathbb{C}}$, we obtain a canonical isomorphism

$$
C(V(X)) \cong D(X)=\bigotimes_{P, \text { out }} D \otimes \bigotimes_{P, \text { in }} D^{O p}
$$


Now for a maximal isotropic subspace $W \in G r_{r e s} \Omega^{1 / 2}(\partial X)_{\mathbb{C}}$ (i.e. a complex structure on $\left.\Omega^{1 / 2}(\partial X)_{\mathbb{R}}\right)$, we have already defined a graded Pfaffian line

$$
P f_{W}(X)
$$

Using Observation 5.23, define a $D(X)$-module

$$
P f(X):=P f_{W_{0}(X)}(W) \otimes P f_{W}(X) .
$$

It is not difficult to verify that this definition does not depend on the choice of $W$, and satisfies the requisite axioms.

Comment. - P. Deligne remarks that the consideration of real structure is not necessary to define a Pfaffian. The construction makes sense on a polarized Hilbertizable infinite-dimensional $\mathbb{C}$-vector space $H$ with non-degenerate symmetric bilinear form $B$. It then makes sense to say whether $H$ is even or odd dimensional. If $H$ is even-dimensional, then for a maximal $B$-isotropic closed subspace $W \subset H, W \in G r_{\text {res }}(H), B$ defines an iso

$$
H / W \cong W^{*} .
$$

A splitting

$$
H / W \rightarrow H
$$

whose target is isotropic specifies a real structure (i.e. an antilinear complex conjugation operator) on $H$, but any two such splittings differ by a skew map $W^{*} \rightarrow W$ which is HilbertSchmidt if the image of the splitting is in the restricted Grassmanian of the opposite polarization.

For two maximal isotropic closed subspaces $W, W_{0}$, we can then define a Pfaffian line $P f_{W_{0}}(W)$. More generally, for $W_{1} \in G r_{r e s}(H)$ isotropic, one can define a super-central simple $\mathbb{C}$-algebra $C$ as the commutant of the $B$-annihilator of $\Lambda\left(W_{1}\right)$, considered as a right $C(H)$-module, and one then has a canonical irreducible $C$-module $P f_{W_{1}}(W)$.

This corresponds to the fact that, if $H$ is finite-dimensional, the subgroup of $G L(H)$ preserving $B$ is the complex orthogonal group, which has the same homotopy type as the real orthogonal group, and hence has a Spin-central extension. In the infinite-dimensional case, a similar discussion applies.

\section{Trivial $\mathbb{C}^{\times}$-central extensions}

In this section, we shall classify trivial $\mathbb{C}^{\times}$-central extensions of $\mathcal{C}^{\text {top }}, \mathcal{C}_{\text {spin }}^{\text {top }}$.

THEOREM 6.1. - The group of isomorphism classes of $\mathbb{C}^{\times}$-central extensions of $\mathcal{C}^{\text {top }}, \mathcal{C}_{\mathbb{Z} / 2}^{\text {top }}$ are isomorphic to $\mathbb{Z} / 2$, generated by a $\mathbb{C}^{\times}$-central extension in which the degree of the line associated to a surface with d components is $d \bmod 2$.

Proof of the $\mathcal{C}$ case. - First we will calculate the possible degrees of lines associated to conformal surfaces. (For brevity, we will sometimes refer to this degree as the degree of the surface associated with the given central extension, or the degree of the central extension on the surface.) Surfaces can be cut up into disks, annuli and pairs of pants. Annuli with 1 incoming and 1 outgoing component form a semigroup, and hence must have degree 0 . Therefore, the degree of all surfaces is determined by the degree of the outgoing disk $D_{+}$and incoming disk $D_{-}$. 
Claim 6.2. - In a $\mathbb{C}^{\times}$-central extension of $\mathcal{C}^{\text {top }}$, the degrees of $D_{+}$and $D_{-}$are equal $(\bmod 2)$.

Proof. - Suppose, say, that $\operatorname{deg}\left(D_{+}\right)=1, \operatorname{deg}\left(D_{-}\right)=0$. Let $A_{++}$(resp. $A_{--}$be an annulus with two outgoing (resp. incoming) boundary components. Then $A_{++}$and $A_{--}$have nontrivial automorphisms $\alpha_{++}$and $\alpha_{--}$, switching the boundary components. However, since automorphisms of $\mathbb{P}^{1}$ are trivial in $\mathcal{C}^{\text {top }}$, and $\mathbb{P}^{1}$ can be glued from $D_{+}, D_{+}, A_{--}$(resp. $\left.D_{-}, D_{-}, A_{++}\right)$in a way equivariant with respect to $\alpha_{--}$(resp. $\alpha_{++}$) and a switch of disks, we conclude that the sign induced by $\alpha_{++}$on its central extension line is +1 , while the sign induced by $\alpha_{--}$is -1 . But now an elliptic curve can be glued from $A_{++} \coprod A_{--}$equivariantly with respect to $\alpha_{++}, \alpha_{--}$, indicating that the automorphism (which is trivial in $\mathcal{C}^{\text {top }}$ ) induces multiplication by -1 .

Note that the generator is obtained as the tensor product of the two kinds of determinants mentioned above (resulting from shifting the polarization of $L^{2}\left(S^{1}\right)$ by index 1 ). Thus, the proof of Theorem 6.1 will follow from the following.

CLAIM 6.3. - Every $\mathbb{C}^{\times}$-central extension of $\mathcal{C}^{\text {top }}$ in which all degrees are 0 (we will call this a degree 0 extension) is isomorphic to the unit central extension $I$.

Proof. - Let $L$ be a degree $0 \mathbb{C}^{\times}$-central extension of $\mathcal{C}^{\text {top }}$. For each connected component $[* / S]$ of $\mathcal{C}^{t o p}$, we shall first prove that the modular group $S$ acts trivially on $L$. To this end, note that by use of sewing, it suffices to show that the switch of boundary components acts trivially on the annulus $A_{++}$. Recall the automorphism $\alpha_{++}$of $A_{++}$switching boundary components. Then, however, we can sew two copies of $D_{-}$on the two boundary components of $A_{++}$, obtaining a copy of $\mathbb{P}^{1}$, with a non-trivial automorphism obtained by sewing the automorphism $\alpha_{++}$with a switch of the $D_{-}$'s. But by our assumptions, the switch of $D_{-}$'s induces 1 on $L$, and so does the automorphism of $\mathbb{P}^{1}$. Hence, the automorphism $\alpha_{++}$induces 1 on $L$ also. The treatment of $\alpha_{--}$ is analogous.

Now we shall construct an isomorphism $L \cong I$. First choose isomorphisms $L_{D_{+}} \cong I \cong L_{D_{-}}$ arbitrarily. Sewing then forces the isomorphism $L \cong 1$ on annuli and pairs of pants, and therefore on every surface in $\mathcal{C}^{t o p}$. We must show that the isomorphism thus defined is consistent. This amounts to saying that if we cut a surface $X$ into disks, annuli and pairs of pants in two different ways, the two resulting isomorphisms $L_{X} \cong 1$ coincide. To this end, we first consider surfaces $X$ of genus 0 . (The genus of a surface with boundary is defined as the genus of the surface obtained by sewing on disks.) Note that our choices specify an isomorphism $L_{\mathbb{P}^{1}} \cong 1$ and we can define $L_{X}$ simply by requiring that it be consistent with attaching disks to all boundary components of $X$ (which gives $\mathbb{P}^{1}$ ). This is obviously compatible with sewing in the category of surfaces of genus 0 (the reason this argument does not work in $\mathcal{C}$ is that there are different kinds of disks). Now if we have a connected surface $X$ of genus $>0$, cut $X$ into Riemann surfaces of genus 0 along non-separating curves. Note that there is an ambiguity in such cutting due to possible permutations of boundary components. However, we have proven that such permutations induce the identity on $L$.

Proof of the $\mathcal{C}_{\text {spin }}$ case. - Recall that for a $\mathbb{C}^{\times}$-central extension we have its degree which is a function assigning to each surface in the respective category an element of $\mathbb{Z} / 2$.

Following similar arguments as in the case of $\mathcal{C}$, one sees that the degrees of all elements of $\mathcal{C}_{\text {spin }}^{\text {top }}$ with respect to a $\mathbb{C}^{\times}$-central extension are determined by degrees of the following surfaces:

(1) $\operatorname{deg}\left(D_{+}\right)=\operatorname{deg}\left(D_{-}\right)$(there is only one possible Spin-structure).

(2) $\operatorname{deg}(L)$ where $L$ is a periodic annulus with one inbound and one outbound boundary component, reversing spin.

(3) $\operatorname{deg}(P)$ where $P$ is a periodic annulus with two inbound periodic boundary components. 
We have shown in (4.30) that

$$
\operatorname{deg}(L)=0,
$$

even for $\mathbb{C}^{\times}$-central extensions of $\mathcal{C}_{\text {spin }}$, and, in fact, the line corresponding to $L$ is canonically trivialized. Thus, to show that we have the same possible degrees as in the case of $\mathcal{C}^{\text {top }}$, it remains to show that

$$
\operatorname{deg}(P)=0
$$

To this end, suppose that

$$
\operatorname{deg}(P) \neq 0
$$

for a $\mathcal{C}^{\times}$-central extension $D$ of $\mathcal{C}_{\text {spin }}^{\text {top }}$. Consider a genus 0 Spin-surface $X$ with four outbound boundary components. We have seen above in Lemma 2.24 that in $\mathcal{C}_{\text {spin }}^{\text {top }}$, there is an automorphism $\alpha$ of $X$ which induces the permutation (12)(34) on boundary components. We can sew $\alpha$ with a switch of two copies of $P$ to get an automorphism of a genus 2 closed surface, which is trivial in $\mathcal{C}_{\text {spin }}^{\text {top }}$. Because of this, (6.6) implies that $\alpha$ induces -1 on $D_{X}$. By sewing, it follows that there are permutations in $S^{s p i n}$ of boundary components of a genus 0 Spin-surface $Y$ with six outbound periodic boundary components which induce -1 on $D_{Y}$. Thus, by Lemma 2.24, we would obtain a non-trivial homomorphism

$$
A_{6} \rightarrow \mathbb{C}^{\times}
$$

which is a contradiction. Thus, (6.5) is proven.

We have reduced our task to proving

Lemma 6.7. - All degree $0 \mathbb{C}^{\times}$-central extensions of $\mathcal{C}_{\text {spin }}^{\text {top }}$ are isomorphic to the unit $\mathbb{C}^{\times}$-central extension $I$.

Consider a $\mathbb{C}^{\times}$-central extension $M$ of $\mathcal{C}_{\text {spin }}^{\text {top }}$. First note that our proof of (6.5) also implies that the group $S^{\operatorname{spin}}\left(X_{0}\right)$ acts trivially on $M_{X_{0}}$.

We shall now define a natural isomorphism $M \cong I$ on Spin-surfaces of genus 0 , compatible with sewing: the trivialization is defined arbitrarily on $D_{+}, D_{-}$and annulus with two outbound periodic boundary components. It is then specified uniquely on all pairs of pants, and every Spin-surface $X$ of genus 0 can be cut up into pairs of pants.

The cutting is determined by matching (or dividing into pairs) the periodic boundary components of $X$. Observe carefully that the choice of matching does matter slightly: any two matchings are carried one to another by a permutation whose sign is well defined. Suppose, for simplicity, that $X$ is connected and has outbound periodic boundary components only. Then $S^{\operatorname{spin}}(X)$ is the alternating group, so the order of boundary components of $X$ is defined up to sign, and compatibility of order with sewing (up to sign) in $\mathcal{C}^{\text {spin }}$ shows that there is a 'positive' choice of matching which we should use for our trivialization. Reversing the sign of the order of boundary components changes $X$ into a different (although isomorphic) surface $X^{\prime}$. Comparing the trivializations of $M_{X}$ and $M_{X^{\prime}}$ gives a trivialization of $M_{L}$ where $L$ is the periodic annulus with one inbound and one outbound boundary component reversing spin.

Claim 6.8. - Consider the elliptic curve $E$ with Spin-structure of Kervaire invariant 0 , and the ratio $\lambda$ of the images of the canonical elements $\lambda_{L}, \lambda_{A}$ of the $\mathbb{C}^{\times}$-central extension $M$ of $\mathcal{C}_{\text {spin }}^{\text {top }}$ on the periodic and antiperiodic annuli $L, A$ ( $L$ reversing spin - see (4.30)) obtained by cutting E along a periodic (resp. antiperiodic) curve. Then $\lambda=1$. 
Proof. - Consider a surface $Q$ obtained from $\mathbb{P}^{1}$ by cutting out four disks $D_{1}, \ldots, D_{4}$, where $D_{1}, D_{4}$ are inbound, $D_{2}, D_{3}$ are outbound. Choose a Spin-structure on $Q$ where $D_{1}, D_{2}$ are periodic, $D_{3}, D_{4}$ are antiperiodic. There is a simple curve $c$ in $Q$ separating $D_{1}$ and $D_{3}$ from $D_{2}$ and $D_{4}$, and $c$ is periodic. Now construct a genus 2 Spin-surface $X$ by sewing $D_{1}$ and $D_{2}$ together into a curve $\lambda$, and $D_{3}, D_{4}$ together into a curve $\mu$. We shall need one more simple closed curve $\nu$ in $X$ obtained from a simple line in $Q$ joining a point $x \in \partial D_{3}$ with a point $y \in \partial D_{4}$ such that $x, y$ correspond to the same point on $\mu$. We can choose spins on $\partial D_{3}, \partial D_{4}$ in such a way that the curve $\nu$ is periodic in $X$.

Then we make two observations: First of all, by cutting $X$ along $\lambda, \mu$ or $\lambda, c$ gives us two genus 0 Spin-surfaces $e, f$ on which $M$ therefore has canonical elements. However, the images of these two elements in $M_{X}$ coincide, since they must coincide with the image of the canonical element in $M$ of the (disconnected) genus 0 surface obtained by cutting $X$ along the three disjoint simple curves $\lambda, c, \mu$.

The second observation is that there is an automorphism of $X$ in $\mathcal{C}_{\text {spin }}^{\text {top }}$ which carries $\lambda, c$ in this order onto $\lambda, \nu$ : to this end, simply observe that in $\mathcal{C}_{\text {spin }}^{\text {top }}$, the genus 0 Spin-surfaces obtained from $X$ by cutting along $\lambda, \nu$ and $\lambda, c$ are isomorphic.

By the triviality of actions of $\mathcal{C}_{\text {spin }}^{\text {top }}$-modular groups, the image in $M_{X}$ of the canonical element obtained from $M$ on the genus 0 surface obtained by cutting along $\lambda, \nu$ is now equal to the element of $M_{X}$ obtained from the canonical element of the $M$ of the surface obtained by cutting along $\lambda, \mu$. But we see that if $\kappa$ is a simple (antiperiodic) curve in $Q$ separating $D_{1}$ and $D_{2}$ from $D_{3}$ and $D_{4}$, then $X$ is the connected sum, along $\kappa$, of two elliptic curves in one of which $\mu, \nu$ are positioned as in the statement of claim, and the other one of which contains $\lambda$.

Proof of Lemma 6.7. - In view of what we already showed, it suffices to prove that for a connected Spin-surface $X$, the trivialization of $M_{X}$ obtained by cutting $X$ along disjoint simple curves along disjoint simple curves into a connected genus 0 surface does not depend on the choice of curves. Clearly, it suffices to consider the case when $X$ is closed of genus $n$. Consider a set $R$ of $n$ disjoint simple curves in $X$ (thus specifying a maximal isotropic subspace of the canonical bilinear form on $H^{1}(X, \mathbb{Z} / 2)$ ).

By a hyperbolic switch, we shall mean the following modification of $R$ : Expressing $X$ as a connected sum

$$
X=Y \sharp E
$$

of $Y$ and an elliptic curve $E$ (along a curve disjoint with $R$ ) such that $E$ is diffeomorphic to $r \times s, r \in R$, then replace $R$ by

$$
R^{\prime}=(R-\{r\}) \cup\{s\} .
$$

In view of Claim 6.8, our statement then follows from the following result.

LEMMA 6.9. - Any two sets $R, R^{\prime}$ of $n$ disjoint simple curves in $X$ can be carried one onto another by a sequence of hyperbolic switches and elements of the Spin-mapping class group of $X$.

Proof. - Let $R, R^{\prime}$ be as above. By cutting along $R$ (resp. $R^{\prime}$ ) and sewing back, we see that we can write

$$
R=\left\{r_{1}, \ldots, r_{n}\right\}, \quad R^{\prime}=\left\{r_{1}^{\prime}, \ldots, r_{n}^{\prime}\right\},
$$

such that $X$ is a connected sum of elliptic curves $E_{i}$ (resp. $E_{i}^{\prime}$ ) diffeomorphic to $r_{i} \times s_{i}$ (resp. $r_{i}^{\prime} \times s_{i}^{\prime}$ ) for some $s_{i}, s_{i}^{\prime}$. (This specifies a selection of two hyperbolic bases of $H^{1}(X, \mathbb{Z} / 2)$.) 
Now consider the numbers $k$ (resp. $k^{\prime}$ ) of the elliptic curves $E_{i}$ (resp. $E_{i}^{\prime}$ ) which have Kervaire invariant 1 . The parity of these numbers is the Kervaire invariant of the Spin-structure on $X$, and hence

$$
k \equiv k^{\prime} \bmod 2 .
$$

Note that if $k=k^{\prime}$, we are done.

Thus, it suffices to show how to modify the number $k$ by 2 by means of hyperbolic switches. To this end, it suffices to consider $X$ of genus 2 . Without loss of generality, we may assume that $r_{1}, r_{2}$ are periodic. We must show that $X$ can be expressed in two ways as a connected sum of two elliptic curves $E_{1}, E_{2}, E_{1}^{\prime}, E_{2}^{\prime}$ where $E_{i}$ have Kervaire invariant 0 and $E_{i}^{\prime}$ have Kervaire invariant 1 , and $E_{i}=r_{i} \times s_{i}, E_{i}^{\prime}=r_{i} \times s_{i}^{\prime}$. To this end, cut $X$ along $r_{1}, r_{2}$, creating a genus 0 surface with four periodic boundary components. Arranging the four boundary components in the corners of a square, such that the pairs of components to be sewn are facing diagonally, the curves $s_{i}$ are obtained by connecting one diagonal pair of corners by a straight line, and the other by a disjoint line. The curves $s_{i}^{\prime}$ are obtained in a similar way, switching the roles of the diagonals.

Remark. - The discussion of the case of $\mathcal{C}_{\text {spin }}^{c, t o p}$ is completely analogous, with the exception that degree $0 \mathbb{C}^{\times}$-central extensions $D$ of $\mathcal{C}_{\text {spin }}^{c, t o p}$ have an additional invariant, namely the map on $D_{B}$ induced by an isomorphism

$$
\phi: B \rightarrow B
$$

switching boundary components (see (4.30)). Note that

$$
\phi^{4}=1
$$

while $\phi^{2}$ is spin reversal on $B$. However, note that we must have

$$
D_{\phi^{2}}=I d
$$

taking a connected sum of two copies of $B$, we obtain a genus 0 Spin-surface $X$ with 4 outbound periodic boundary components. Reversing spin on two of them, considered as an element of $S_{C}(X)$, cannot induce -1 , since performing such reversal on components 1,2 then 2,3 then 3,1 gives $I d$.

Thus, $\phi$ induces \pm 1 . Now it turns out that a degree $0 \mathbb{C}^{\times}$-central extension of $\mathcal{C}_{\text {spin }}^{c}$ on which $\phi$ induces -1 does exist: It is obtained as the conformal anomaly of the tensor product of the two variants of the Spin-CFT $\mathcal{F}\left(\Omega^{1 / 2}\right)$ where in one we choose

$$
\Omega_{+}^{1 / 2}\left(S_{P}^{1}\right)=\left\langle z^{n} d u^{1 / 2} \mid n \geqslant 0\right\rangle, \quad z=\mathrm{e}^{2 \pi i u},
$$

and in the other

$$
\Omega_{+}^{1 / 2}\left(S_{P}^{1}\right)=\left\langle z^{n} d u^{1 / 2} \mid n>0\right\rangle .
$$

The rest of the argument is analogous, thus giving

THEOREM 6.10. - The group of $(\mathbb{Z} / 2$-graded $) \mathbb{C}^{\times}$-central extensions of $\mathcal{C}_{\text {spin }}^{c \text {,top }}$ is isomorphic to $\mathbb{Z} / 2 \times \mathbb{Z} / 2$ where one generator has degree $d \bmod 2$ on a surface with $d$ boundary components, and the other generator $D$ has degree 0 , but satisfies

$$
D_{\phi}=-1 \text {. }
$$


Analogous discussions can be made for $\mathcal{C}_{\mathbb{Z} / 2}^{\text {top }}, \mathcal{C}_{\mathbb{Z} / 2}^{c, \text { top }}$.

\section{Some basic facts on level 2 modular transformations}

In this section, we shall review some classical facts which are of interest to us. For the most part, S. Lang [15], Chapter 18, is a sufficient reference for our purposes, with his older references filling in all the details.

We begin with Dedekind's $\eta$-function

$$
\eta(\tau)=q^{1 / 24} \prod_{n>0}\left(1-q^{n}\right)
$$

where, as always, $q=\mathrm{e}^{2 \pi i \tau}$. To discuss the automorphic properties of $\eta$, let $\Gamma(1)=P S L_{2}(\mathbb{Z})$. Then $\Gamma(1) \cong(\mathbb{Z} / 2 \mathbb{Z}) *(\mathbb{Z} / 3 \mathbb{Z})$, so $\Gamma(1)$ has unique non-trivial central extensions by $\mathbb{Z} / 2$, $\mathbb{Z} / 4$, respectively. The $\mathbb{Z} / 2$-central extension is $S L_{2}(\mathbb{Z})$. We denote the $\mathbb{Z} / 4$-central extension by $\widehat{S L_{2}(\mathbb{Z})}$. This group can be described as the set of all pairs

$$
\left\{(g, f) \mid g=\left(\begin{array}{ll}
a & b \\
c & d
\end{array}\right), a d-b c=1, f: \mathbb{H} \rightarrow \mathbb{C} \text { holomorphic, } f(\tau)^{2}=c \tau+d\right\}
$$

where $\mathbb{H}$ is the upper halfplane in $\mathbb{C}$. The composition is defined by

$$
(g, f) \circ\left(g^{\prime}, f^{\prime}\right)=\left(g g^{\prime}, f^{\prime \prime}\right)
$$

where

$$
f^{\prime \prime}(\tau)=f^{\prime}(\tau) \cdot f\left(g^{\prime}(\tau)\right) .
$$

Now the abelianization $P S L_{2}(\mathbb{Z}) \rightarrow \mathbb{Z} / 6$ obviously gives an abelianization

$$
\widetilde{S L_{2}(\mathbb{Z})} \rightarrow \mathbb{Z} / 24
$$

We choose a model of this map

$$
u: \widetilde{S L_{2}(\mathbb{Z})} \rightarrow \mu_{24}
$$

( $\mu_{n}$ means the $n$th roots of unity) where

$$
\begin{aligned}
& u\left(\left(\begin{array}{cc}
1 & 1 \\
0 & 1
\end{array}\right), I d\right)=\mathrm{e}^{2 \pi i / 24}, \\
& u\left(\left(\begin{array}{cc}
0 & -1 \\
1 & 0
\end{array}\right), f\right)=\mathrm{e}^{-2 \pi i / 8}
\end{aligned}
$$

where

$$
f(i)=\mathrm{e}^{2 \pi i / 8} .
$$

Now we have for $(g, f) \in \widetilde{S L_{2}(\mathbb{Z})}, g: q \mapsto q^{\prime}$ (we will use this notation to mean $q^{\prime}=\mathrm{e}^{2 \pi i \tau^{\prime}}$, $\left.g(\tau)=\tau^{\prime}\right)$

$$
\eta\left(\tau^{\prime}\right)=f(\tau) u((g, f)) \eta(\tau)
$$

(see [15], Theorem 6 on p. 253).

$4^{\mathrm{e}}$ SÉRIE - TOME $36-2003-\mathrm{N}^{\circ} 1$ 
Next, we will look at level 2 modular transformations. The group $\Gamma(2) \subset \Gamma(1)$ is the subgroup consisting of all elements $g$ represented by $\left(\begin{array}{ll}a & b \\ c & d\end{array}\right)$, where $2|b, 2| c$. This can be interpreted as follows: an element $g \in \Gamma(1)$ defines, up to sign, an isomorphism of elliptic curves

$$
i(g): \mathbb{C} /[\tau, 1] \cong \mathbb{C} /[g(\tau), 1]
$$

(Here, and below, $\left[\omega_{1}, \omega_{2}\right]$ denotes the lattice spanned by $\omega_{1}, \omega_{2}$.) The map $i(g)$ is simply $\frac{1}{c \tau+d}$. Now $\Gamma(2)$ consists of all $g \in \Gamma(1)$ for which $i(g)$ preserves each of the points $1 / 2, \tau / 2$, $(1+\tau) / 2 \in \mathbb{C} /[\tau, 1]$. Then $\Gamma(2)$ is, in fact, a degree 6 subgroup of $\Gamma(1)$. It is a free group, with free generators $\left(\begin{array}{ll}1 & 2 \\ 0 & 1\end{array}\right)$ and $\left(\begin{array}{ll}1 & 0 \\ 2 & 1\end{array}\right)$ (hence, the central extensions discussed above split over $\left.\Gamma(2)\right)$. $\Gamma(2)$ acts freely on $\mathbb{H}$, and its fundamental domain is the area in $\mathbb{H}$ bounded by $\{\operatorname{Re}(z)=-1\}$, $\{\operatorname{Re}(z)=1\},\{\|z-1 / 2\|=1 / 2\},\{\|z+1 / 2\|=1 / 2\}$.

It will be beneficial for us to also consider three intermediate groups

$$
\Gamma(2) \subset \Gamma_{1}, \Gamma_{2}, \Gamma_{3} \subset \Gamma(1)
$$

These are defined to be the subgroups of all elements $g \in \Gamma(1)$ for which $i(g)$ preserves the the point $\tau / 2,1 / 2,(\tau+1) / 2$, respectively. These subgroups are of index 3 in $\Gamma(1)$; they are conjugate, and isomorphic to $(\mathbb{Z} / 2 \mathbb{Z}) * \mathbb{Z}$ (the order 2 elements flip the remaining two of the numbers $\tau / 2,1 / 2,(\tau+1) / 2$. For illustration, the fundamental domain, say, of $\Gamma_{2}$, is the area in $H$ bounded by $\{\operatorname{Re}(z)=-1\},\{\operatorname{Re}(z)=0\},\{\|z+1 / 2\|=1 / 2\}$. Note that the non-trivial central extensions of $\Gamma(1)$ by $\mathbb{Z} / 2, \mathbb{Z} / 4$ do pull back to non-trivial extensions of $\Gamma_{i}$. Denote these central extensions by $G_{i}, \widetilde{G}_{i}$, respectively.

Now consider the Weierstrass function

$$
\mathcal{P}(x)=\frac{1}{x^{2}}+\sum_{\omega \in[1, \tau]^{\prime}}\left(\frac{1}{(z-\omega)^{2}}-\frac{1}{\omega^{2}}\right) .
$$

As usual, for a lattice $L, L^{\prime}=L-\{0\}$. We denote

$$
e_{1}=\mathcal{P}(\tau / 2), \quad e_{2}=\mathcal{P}(1 / 2), \quad e_{3}=\mathcal{P}((\tau+1) / 2),
$$

and also

$$
\begin{array}{ll}
P_{0}=\prod_{n=1}^{\infty}\left(1-q^{n}\right), & P_{1}=\prod_{n=1}^{\infty}\left(1-q^{n-1 / 2}\right), \\
P_{2}=\prod_{n=1}^{\infty}\left(1+q^{n}\right), & P_{3}=\prod_{n=1}^{\infty}\left(1+q^{n-1 / 2}\right) .
\end{array}
$$

Following [15], p. 251, we have

$$
\begin{aligned}
& \left(e_{2}-e_{1}\right)^{1 / 4}=\sqrt{\pi} P_{0} P_{3}^{2}, \\
& \left(e_{2}-e_{3}\right)^{1 / 4}=\sqrt{\pi} P_{0} P_{1}^{2}, \\
& \left(e_{3}-e_{1}\right)^{1 / 4}=\sqrt{\pi} 2 q^{1 / 8} P_{0} P_{2}^{2} .
\end{aligned}
$$


For example,

$$
\begin{aligned}
q^{1 / 12} 2 \prod_{n>0}\left(1+q^{n}\right)^{2} & =\frac{\sqrt{\pi} 2 q^{1 / 8} P_{0} P_{2}^{2}}{\sqrt{\pi} q^{1 / 8} P_{0}} \\
& =q^{1 / 12} \frac{\left(e_{3}-e_{1}\right)^{1 / 4}}{\sqrt{\pi} q^{1 / 8} \prod_{n=1}^{\infty}\left(1-q^{n}\right)}=\frac{\left(e_{3}-e_{1}\right)^{1 / 4}}{\sqrt{\pi} \eta(\tau)}
\end{aligned}
$$

On the other hand, we have

$$
q^{1 / 12} \prod_{n>0}\left(1+q^{n}\right)^{2}=\frac{q^{1 / 6} \prod_{n>0}\left(1-q^{2 n}\right)^{2}}{q^{1 / 12} \prod_{n>0}\left(1-q^{n}\right)^{2}}=\frac{\eta(2 \tau)^{2}}{\eta(\tau)^{2}} .
$$

By (7.2), the modularity properties of the right hand side of (7.5) can now be computed. Define

$$
\alpha\left(\left(\begin{array}{ll}
a & b \\
c & d
\end{array}\right)\right)=\left(\begin{array}{cc}
a & 2 b \\
c / 2 & d
\end{array}\right) .
$$

Thus, for $g \in \Gamma(2)$ (actually, $g \in G_{2}$ ),

$$
\frac{\left(q^{\prime}\right)^{1 / 12} \prod_{n>0}\left(1+\left(q^{\prime}\right)^{n}\right)^{2}}{q^{1 / 12} \prod_{n>0}\left(1+q^{n}\right)^{2}}=\frac{u^{2}(\alpha(g))}{u^{2}(g)}=: v_{31}(g) .
$$

Here the notation $v_{31}(g)$ refers to the formula (7.4): We will later be interested in the transforms of this formula by level 1 modular transformations permuting the $e_{i}$ 's. For now, explicitly, for $g \in \Gamma(2), g: \tau \mapsto \tau^{\prime}, g: e_{i} \mapsto e_{i}^{\prime}$,

$$
\frac{\frac{\left(e_{3}^{\prime}-e_{1}^{\prime}\right)^{1 / 4}}{\eta\left(\tau^{\prime}\right)}}{\frac{\left(e_{3}-e_{1}\right)^{1 / 4}}{\eta(\tau)}}=v_{31}(g)
$$

By (7.1), $v_{31}$ can now be computed. First, we have,

$$
\begin{aligned}
& u^{2}\left(\left(\begin{array}{ll}
1 & 1 \\
0 & 1
\end{array}\right)\right)=\mathrm{e}^{2 \pi i / 12} \\
& u^{2}\left(\left(\begin{array}{cc}
0 & -1 \\
1 & 0
\end{array}\right)\right)=-i
\end{aligned}
$$

Consequently,

$$
\begin{aligned}
& v_{31}\left(\begin{array}{ll}
1 & 0 \\
2 & 1
\end{array}\right)=\frac{u^{2}\left(\left(\begin{array}{ll}
1 & 0 \\
1 & 1
\end{array}\right)\right)}{u^{2}\left(\left(\begin{array}{ll}
1 & 0 \\
2 & 1
\end{array}\right)\right)}=\frac{\mathrm{e}^{-2 \pi i / 12}}{\mathrm{e}^{-2 \pi i / 6}}=\mathrm{e}^{2 \pi i / 12}, \\
& v_{31}\left(\begin{array}{ll}
1 & 1 \\
0 & 1
\end{array}\right)=\frac{u^{2}\left(\left(\begin{array}{ll}
1 & 2 \\
0 & 1
\end{array}\right)\right)}{u^{2}\left(\left(\begin{array}{ll}
1 & 1 \\
0 & 1
\end{array}\right)\right)}=\frac{\mathrm{e}^{2 \pi i / 6}}{\mathrm{e}^{2 \pi i / 12}}=\mathrm{e}^{2 \pi i / 12} .
\end{aligned}
$$

We will be interested in calculating the transforms $v_{i j}$ of $v_{31}$ by level one modular transformations which carry $e_{3} \mapsto e_{i}, e_{1} \mapsto e_{j}$. First, however, let us record the corresponding transforms of (7.4): We have

$$
q^{-1 / 24} \prod_{n \geqslant 0}\left(1+q^{n+1 / 2}\right)^{2}=\frac{\left(e_{2}-e_{1}\right)^{1 / 4}}{q^{1 / 24} \sqrt{\pi} \prod_{n=1}^{\infty}\left(1-q^{n}\right)}=\frac{\left(e_{2}-e_{1}\right)^{1 / 4}}{\sqrt{\pi} \eta(\tau)}
$$


and, symmetrically,

$$
q^{-1 / 24} \prod_{n \geqslant 0}\left(1-q^{n+1 / 2}\right)^{2}=\frac{\left(e_{2}-e_{3}\right)^{1 / 4}}{\sqrt{\pi} \eta(\tau)} .
$$

Now let, for $g \in \Gamma(2), g: \tau \mapsto \tau^{\prime}, g: e_{i} \mapsto e_{i}^{\prime}$,

$$
\frac{\frac{\left(e_{i}^{\prime}-e_{j}^{\prime}\right)^{1 / 4}}{\eta\left(\tau^{\prime}\right)}}{\frac{\left(e_{i}-e_{j}\right)^{1 / 4}}{\eta(\tau)}}=v_{i j}(g)
$$

To calculate $v_{21}$, we note that

$$
v_{21}(g)=v_{31}\left(\left(\begin{array}{ll}
1 & 0 \\
1 & 1
\end{array}\right) g\left(\begin{array}{cc}
1 & 0 \\
-1 & 1
\end{array}\right)\right)
$$

and hence

$$
\begin{aligned}
& v_{21}\left(\begin{array}{ll}
1 & 0 \\
2 & 1
\end{array}\right)=v_{31}\left(\begin{array}{ll}
1 & 0 \\
2 & 1
\end{array}\right)=\mathrm{e}^{2 \pi i / 12} \\
& v_{21}\left(\begin{array}{ll}
1 & 2 \\
0 & 1
\end{array}\right)=v_{31}\left(\begin{array}{ll}
-1 & 2 \\
-2 & 3
\end{array}\right)=\mathrm{e}^{-2 \pi i / 12} \\
& v_{21}\left(\begin{array}{cc}
2 & 1 \\
-1 & 0
\end{array}\right)=v_{31}\left(\begin{array}{ll}
1 & 1 \\
0 & 1
\end{array}\right)=\mathrm{e}^{2 \pi i / 12}
\end{aligned}
$$

Similarly,

$$
v_{23}(g)=v_{21}\left(\left(\begin{array}{cc}
1 & 1 \\
0 & 1
\end{array}\right) g\left(\begin{array}{cc}
1 & -1 \\
0 & 1
\end{array}\right)\right)
$$

and hence

$$
\begin{aligned}
& v_{23}\left(\begin{array}{ll}
1 & 2 \\
0 & 1
\end{array}\right)=v_{21}\left(\begin{array}{ll}
1 & 2 \\
0 & 1
\end{array}\right)=\mathrm{e}^{-2 \pi i / 12} \\
& v_{23}\left(\begin{array}{ll}
1 & 0 \\
1 & 1
\end{array}\right)=v_{21}\left(\begin{array}{cc}
2 & -1 \\
1 & 0
\end{array}\right)=\mathrm{e}^{-2 \pi i / 12} .
\end{aligned}
$$

We conclude this section by remarking that one can use (7.4), (7.9), (7.10), (7.8), (7.12) and (7.13) to obtain, for $g \in \Gamma(2), g: e_{i} \mapsto e_{i}^{\prime}$,

$$
\frac{\frac{\left(e_{2}^{\prime}-e_{1}^{\prime}\right)^{1 / 4}}{\left(e_{3}^{\prime}-e_{1}^{\prime}\right)^{1 / 4}}}{\frac{\left(e_{2}-e_{1}\right)^{1 / 4}}{\left(e_{3}-e_{1}\right)^{1 / 4}}}=\frac{v_{21}(g)}{v_{31}(g)}= \begin{cases}1 & \text { for } g=\left(\begin{array}{ll}
1 & 0 \\
2 & 1
\end{array}\right), \\
\mathrm{e}^{-2 \pi i / 4} & \text { for } g=\left(\begin{array}{ll}
1 & 2 \\
0 & 1
\end{array}\right),\end{cases}
$$

and

$$
\frac{\frac{\left(e_{2}^{\prime}-e_{3}^{\prime}\right)^{1 / 4}}{\left(e_{3}^{\prime}-e_{1}^{\prime}\right)^{1 / 4}}}{\frac{\left(e_{2}-e_{3}\right)^{1 / 4}}{\left(e_{3}-e_{1}\right)^{1 / 4}}}=\frac{v_{23}(g)}{v_{31}(g)}= \begin{cases}\mathrm{e}^{-2 \pi i / 4} & \text { for } g=\left(\begin{array}{ll}
1 & 0 \\
2 & 1
\end{array}\right), \\
\mathrm{e}^{-2 \pi i / 4} & \text { for } g=\left(\begin{array}{ll}
1 & 2 \\
0 & 1
\end{array}\right) .\end{cases}
$$

This could, of course, also be deduced directly, since

$$
\frac{e_{2}-e_{3}}{e_{3}-e_{1}}, \quad \frac{e_{2}-e_{1}}{e_{3}-e_{1}}
$$


are images under level 1 modular transformations of

$$
\lambda=\frac{e_{2}-e_{3}}{e_{1}-e_{3}},
$$

which is the generator of the field of modular functions of level 2 (see [15], Section 18.6).

\section{Modularity in holomorphic Spin- and Sqrt-conformal field theories}

We will start with the case of Sqrt-CFT's, which is slightly simpler. Note that a Sqrt-CFT is in particular a CFT, so the modularity formula for $Z(\tau)$ is given by (3.3) above. We will now treat the case of $Z^{-}(\tau)$. Define $\varrho: \Gamma_{2} \rightarrow \mu_{4} \subset \mathbb{C}^{\times}$by the formula

$$
\varrho\left(\begin{array}{ll}
1 & 0 \\
2 & 1
\end{array}\right)=\mathrm{e}^{2 \pi i / 4}, \quad \varrho\left(\begin{array}{ll}
1 & 1 \\
0 & 1
\end{array}\right)=1 .
$$

THEOREM 8.1. - Let $g \in \Gamma_{2}$, let $g(\tau)=\tau^{\prime}, q=\mathrm{e}^{2 \pi i}, q^{\prime}=\mathrm{e}^{2 \pi i \tau^{\prime}}$. Then, for a Sqrt-CFT,

$$
\frac{Z^{-}\left(\tau^{\prime}\right)}{Z^{-}(\tau)}=\frac{\left(q^{\prime}\right)^{c / 24}}{q^{c / 24}} u(g)^{-c} \varrho(g)^{-H}
$$

where $c$ is the central charge, and $H=8 h_{A} \in \mathbb{Z} / 4 \mathbb{Z}$.

Proof. - First, note that gluing the boundary components of $\left(A_{q},-1\right) \in \mathcal{A}_{P} \subset \mathcal{C}_{\mathbb{Z} / 2}$, we obtain the elliptic curve $\mathbb{C} /[\tau, 1]$ with the $S q r t$-structure $[1 / 2]-[0]$. Thus, the group of symmetry is $\Gamma_{2}$.

To see this, study the divisor of the theta-function $\theta$ which would satisfy

$$
\theta(u+1)=\theta(u), \quad \theta(u+\tau)=-\theta(u)
$$

Given the fact that the function $\phi$ has divisor [0] and satisfies

$$
\phi(u+1)=\phi(u), \quad \phi(u+\tau)=-\frac{1}{\mathrm{e}^{2 \pi i u}},
$$

we see that $\theta(u)=\phi(u+1 / 2) / \phi(u)$ satisfies (8.3). Now $\theta$ has divisor $[1 / 2]-[0]$, as claimed.

Now recall that the group of $\mathbb{C}^{\times}$-central extensions of $\mathcal{C}_{\mathbb{Z} / 2}$ is $\mathbb{Z} \oplus \mathbb{Z} / 4 \mathbb{Z}$, spanned by Det and $Q$. Now we have a tensor product of CFT's, and because Det and $Q$ are conformal anomalies of CFT's, so is every $\mathbb{C}^{\times}$-central extension of $\mathcal{C}_{\mathbb{Z} / 2}$. Because, further, the formula (8.2) is clearly preserved by tensor product of CFT's, it suffices to establish the formula for a CFT whose conformal anomaly is $Q$ or Det.

Now in the case of Det, the formula is valid by Corollary 3.4 above, since Det is independent of the gluing.

In the case of $Q$, on the other hand, we have

$$
Q\left(E_{\tau},\left[\frac{1}{2}\right]-[0]\right)=\operatorname{Det} H^{*}\left(E_{\tau},\left[\frac{1}{2}\right]-[0]\right)=\operatorname{Det}(0)=\mathbb{C} .
$$

Therefore, $Q\left(E_{\tau},\left[\frac{1}{2}\right]-[0]\right)$ contains a canonical element 1 which is preserved by the action of $\Gamma_{2}$. Similarly as in the proof of Theorem 3.3 above, we see that the image of the canonical 
element of $Q\left(\left(A_{q},-1\right)\right) \in \mathcal{A}_{P}$ in $Q\left(E \tau,\left[\frac{1}{2}\right]-[0]\right)$ is

$$
Z_{-}(\tau) \cdot 1
$$

where $Z_{-}(\tau)$ is the negative partition function corresponding to the conformal field theory whose conformal anomaly is $Q$. Now (8.4) is equal to

$$
2 \prod_{n \geqslant 0}\left(1+q^{n}\right)^{2} \cdot 1
$$

The modularity properties of this function are determined by (7.4)-(7.7): We have

$$
\frac{Z_{-}\left(\tau^{\prime}\right)}{Z_{-}(\tau)}=v_{31}(g) \cdot \frac{\left(q^{\prime}\right)^{-1 / 12}}{q^{-1 / 12}} .
$$

But now, by (7.8), $v_{31}(g)=u^{2}(g) \cdot \varrho(g)$, and thus (8.5) is equivalent to (8.2) in this case.

Next, we will treat the modularity of Spin-CFT's. In this case, we defined only one partition function $Z$, which is, however, a function of $q^{1 / 2}$. Now note that when investigating the modularity properties of this function, the variable $\tau$ determines the two square roots of $q$ as $\mathrm{e}^{\pi i \tau},-\mathrm{e}^{\pi i \tau}$. By making a choice of the first or second square root, we get, in effect, two different partition functions, which we denote, in this order, by $Z(\tau), Z_{-}(\tau)$. These functions will have different modularity properties.

We will begin with the function $Z_{-}(\tau)$. First, define a function

$$
w: G_{3} \rightarrow \mu_{8} \subset \mathbb{C}^{\times}
$$

by

$$
\begin{aligned}
& w\left(\left(\begin{array}{ll}
1 & 2 \\
0 & 1
\end{array}\right), I d\right)=1 \\
& w\left(\left(\begin{array}{cc}
0 & -1 \\
1 & 0
\end{array}\right), f\right)=\mathrm{e}^{-2 \pi i / 8}
\end{aligned}
$$

where $f(i)=\mathrm{e}^{2 \pi i / 8}$.

THEOREM 8.6. - Let $g \in G_{3}$, let $g(\tau)=\tau^{\prime}, q=\mathrm{e}^{2 \pi i \tau}, q^{\prime}=\mathrm{e}^{2 \pi i \tau^{\prime}}$. Then, for a Spin-CFT,

$$
\frac{Z_{-}\left(\tau^{\prime}\right)}{Z_{-}(\tau)}=\frac{\left(q^{\prime}\right)^{c / 24}}{q^{c / 24}} u(g)^{-c} w(g)^{-H}
$$

where $c$ is the central charge and $H=8 h_{P} \in \mathbb{Z} / 8 \mathbb{Z}$.

Proof. - We will proceed similarly as in the proof of Theorem 8.1. However, because of $c(P)=1$, we have to deal with subgroups of $\widetilde{S L_{2}(\mathbb{Z})}$.

First, one determines in an analogous manner as above (using theta functions) that by gluing the boundary components of $A_{q} \in \mathcal{A}_{A}$ with negative spin, one obtains the elliptic curve $E_{\tau}$ with Spin-structure given by the divisor $\left[\frac{\tau+1}{2}\right]-[0]$. Thus, the group of symmetry is $G_{3}$.

Now recall that the group of holomorphic $\mathbb{C}^{\times}$-central extensions of $\mathcal{C}_{\text {spin }}$ is $\mathbb{Z} \oplus \mathbb{Z} / 4 \mathbb{Z}$, generated by $D e t$ and $P$. Similarly as above, there are Spin-CFT's whose conformal anomaly is any given holomorphic $\mathbb{C}^{\times}$-central extension of $\mathcal{C}_{\text {spin }}$. Thus, referring to tensor product of CFT's, 
it suffices to verify the statement of the theorem for Spin-CFT's with conformal anomalies Det and $P$.

Once again, the case of $D e t$ follows from (3.4) above, since $H=0$. In the case of $P$, once again,

$$
P\left(E_{\tau},\left[\frac{1+\tau}{2}\right]-[0]\right)=\operatorname{Det}\left(H^{*}\left(E_{\tau}, \Omega_{\left[\frac{1+\tau}{2}\right]-[0]}^{1 / 2}\right)\right)=\operatorname{Det}(0)=\mathbb{C},
$$

so $P\left(E_{\tau},\left[\frac{1+\tau}{2}\right]-[0]\right)$ contains a canonical (modular-invariant) element 1 .

Once again, the image of the canonical element of $P\left(A_{q}\right) \in \mathcal{A}_{A}$ in $P\left(E_{\tau},\left[\frac{1+\tau}{2}\right]-[0]\right)$ is

$$
Z_{-}(\tau) \cdot 1
$$

where $Z_{-}(\tau)$ is the partition function of the CFT conformal anomaly defined above with conformal anomaly $P$. The function (8.8) is equal to

$$
\prod_{n \geqslant 0}\left(1+q^{n+1 / 2}\right)^{2} \cdot 1 .
$$

The modularity properties of this function are determined by (7.9), (7.11). Specifically,

$$
\frac{Z_{-}\left(\tau^{\prime}\right)}{Z_{-}(\tau)}=v_{21}(g) \cdot \frac{\left(q^{\prime}\right)^{1 / 24}}{q^{1 / 24}} .
$$

But now by (7.12), $v_{21}(g)=u(g)^{-1} \cdot w(g)^{-1}$, so (8.9) is equivalent to (8.7) for this conformal anomaly.

Finally, we treat the function $Z(\tau)$ in the case of Spin-CFT's. First, define a homomorphism

$$
\omega: G_{1} \rightarrow \mu_{8} \subset \mathbb{C}^{\times}
$$

by

$$
\begin{aligned}
& \omega\left(\left(\begin{array}{ll}
1 & 2 \\
0 & 1
\end{array}\right), I d\right)=1, \\
& \omega\left(\left(\begin{array}{ll}
1 & 0 \\
1 & 1
\end{array}\right), f\right)=\mathrm{e}^{2 \pi i / 8}
\end{aligned}
$$

where $f: \mathbb{H} \rightarrow \mathbb{C}$ such that $f(\tau)^{2}=\tau+1$ is characterized by

$$
f(i)=2^{1 / 4} \mathrm{e}^{2 \pi i / 16} .
$$

TheOREM 8.10. - Let $g \in G_{1}$, let $g(\tau)=\tau^{\prime}, q=\mathrm{e}^{2 \pi i \tau}, q^{\prime}=\mathrm{e}^{2 \pi i \tau^{\prime}}$. Then, for a Spin-CFT,

$$
\frac{Z\left(\tau^{\prime}\right)}{Z(\tau)}=\frac{\left(q^{\prime}\right)^{c / 24}}{q^{c / 24}} u(g)^{-c} \omega(g)^{-H}
$$

where $c$ is the central charge and $H=8 h_{P} \in \mathbb{Z} / 8 \mathbb{Z}$.

Proof. - The proof is isomorphic to the proof of Theorem 8.6. By gluing the boundary components of $A_{q} \in \mathcal{A}_{A}$ with positive spin, one obtains the elliptic curve $E_{\tau}$ with Spin-structure given by the divisor $\left[\frac{\tau}{2}\right]-[0]$. Thus, the group of symmetry is $G_{1}$. 
Once again, it suffices to prove the statement for a CFT whose conformal anomaly is Det or $P$. Once again, the case of $D e t$ follows from (3.4) above. In the case of $P$, one still has

$$
P\left(E_{\tau},\left[\frac{\tau}{2}\right]-[0]\right)=\operatorname{Det}\left(H^{*}\left(E_{\tau}, \Omega_{\left[\frac{\tau}{2}\right]-[0]}^{1 / 2}\right)\right)=\operatorname{Det}(0)=\mathbb{C},
$$

so $P\left(E_{\tau},\left[\frac{\tau}{2}\right]-[0]\right)$ contains a modular-invariant element 1 .

The image of the canonical element of $P\left(A_{q}\right), A_{q} \in \mathcal{A}_{A}$, in $P\left(E_{\tau},\left[\frac{\tau}{2}\right]-[0]\right)$ is

$$
Z(\tau) \cdot 1
$$

where $Z(\tau)$ is the positive partition function of the CFT with conformal anomaly $P$ which we described above. The function (8.12) is equal to

$$
\prod_{n \geqslant 0}\left(1-q^{n+1 / 2}\right)^{2}
$$

The modularity properties of this function are determined by (7.10), (7.11). Specifically,

$$
\frac{Z\left(\tau^{\prime}\right)}{Z(\tau)}=v_{23}(g) \cdot \frac{\left(q^{\prime}\right)^{1 / 24}}{q^{1 / 24}}
$$

But now by (7.13), $v_{23}(g)=u(g)^{-1} \omega(g)^{-1}$, so (8.13) is equivalent to (8.11) in the case of this conformal anomaly.

\section{Acknowledgement}

I am very thankful to P. Deligne for comments, corrections and clarifications, as well as a concrete suggestion of the use of super-Brauer group.

\section{REFERENCES}

[1] Baranov M.A., Schwarz A.S., Multiloop contribution to string theory, Pisma ZhETP 42 (8) (1985) 340 [JETP Lett. 42 (1986) 419].

[2] Browder W., Surgery on Simply Connected Manifolds, in: Ergebnisse der Mathematik und ihrer Grenzgebiete, Vol. 65, Springer-Verlag, 1972.

[3] Deligne P., Le symbole modéré, Inst. Hautes Études Sci. Publ. Math. 73 (1991) 147-181.

[4] Deligne P., Letters to the author, 2000-2001.

[5] Deligne P., Notes on spinors, in: Quantum Fields and Strings: A Course for Mathematicians, Vol. 1, AMS, 1999, pp. 99-136.

[6] Deligne P., Mumford D., The irreducibility of the space of curves of given genus, Inst. Hautes Études Sci. Publ. Math. 36 (1969) 75-108.

[7] Di Francesco P., Mathieu P., Senechal D., Conformal Field Theory, in: Graduate Texts in Contemporary Physics, Springer-Verlag, New York, 1997.

[8] Dong C., Li H., MAson G., Twisted representations of vertex operator algebras, Math. Ann. 310 (3) (1998) 571-600.

[9] FrenKel I., Vertex algebras and algebraic curves, http://xxx.arXiv.org/ps/math.QA/0007054.

[10] Frenkel I., Lepowsky J., Meurman A., Vertex Operator Algebras and the Monster, in: Pure Appl. Math., Vol. 134, Academic Press, Boston, MA, 1988. 
[11] FRIEDAN D., Notes on string theory and two dimensional conformal field theory, in: Green M., Gross D. (Eds.), Workshop on Unified String Theories, ITP Santa Barbara, World Scientific, Singapore, 1985, p. 162.

[12] Friedan D., Martinec E., Shenker S., Conformal invariance, supersymmetry and string theory, Nucl. Phys. B 271 (1986) 93.

[13] GiRaud, Cohomologie non abélienne, in: Grundlehren der math. Wissensch., Vol. 179, SpringerVerlag.

[14] Huang Y.Z., Two-dimensional Conformal Geometry and Vertex Operator Algebras, in: Progress in Mathematics, Vol. 148, Birkhäuser, 1997.

[15] Lang S., Elliptic Functions, 2nd Edition, in: Graduate Texts in Mathematics, Vol. 112, SpringerVerlag, 1987.

[16] Pressley A., Segal G., Loop Groups, Oxford Science Publications, Oxford University Press, 1986.

[17] Luest D., Theisen S., Lectures on String Theory, Lecture Notes in Mathematics Vol. 346, SpringerVerlag.

[18] Quillen D., Determinants of Cauchy-Riemann operators on Riemann surfaces, Funktsional. Anal. $i$ Prilozhen. 19 (1985) 37-41.

[19] Rabin J.M., Super Riemann Surfaces, in: Yau S.T. (Ed.), Mathematical Aspects of String Theory, in: Advanced Series in Mathematical Physics, Vol. 1, World Scientific, 1987.

[20] Rosly A.A., Schwarz A.S., Voronov A.A., Geometry of superconformal manifolds, Comm. Math. Phys. 119 (1988) 129-152.

[21] SEgal G., The definition of conformal field theory, preprint.

[22] Simon B., Trace Ideals and Their Applications, in: London Mathematical Society Lecture Note Series, Vol. 35, Cambridge University Press, 1979.

[23] WaLl C.T.C., Graded Brauer groups, J. Reine Angew. Math. 213 (1963/64) 187-199.

(Manuscrit reçu le 7 mars 2001; accepté, après révision, le 5 mars 2002.)

Igor KRIZ

University of Michigan,

Department of Mathematics, 2072 East Hall,

525 East University Avenue,

Ann Arbor, MI, 48109-1109, USA

E-mail: ikriz@math.lsa.umich.edu 